$$
\text { DOE/CS/40 341-T7 }
$$

\title{
BLACK LIQUOR GASIFICATION PHASE 2D FINAL REPORT
}

\author{
PREPARED FOR: \\ CHAMPION INTERNATIONAL \\ STAMFORD, CONNECTICUT \\ Work performed under \\ Subcontract STR/DOE-12 \\ of Contract DE-AC05-80CS40341
}

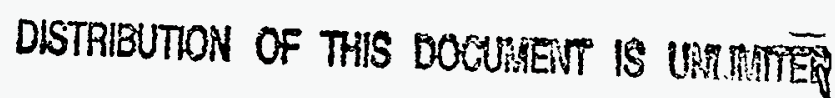

Rockwell International

Rocketdyne Division

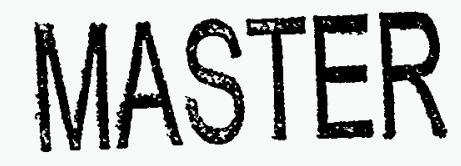


THIS REPORT MAY NOT BE PUBLISHED WITHOUT THE APPROVAL OF THE DOE OFFICE OF PATENT COUNSEL

\section{DISCLAIMER}

This report was prepared as an account of work sponsored by an agency of the United States Government. Neither the United States Government nor any agency thereof, nor any of their employees, nor any of their contractors, subcontractors, or their employees, makes any warranty, express or implied, or assumes any legal liability or responsibility for the accuracy, completeness, or usefulness of any information, apparatus, product, or process disclosed, or represents that its use would not infringe privately owned rights. Reference herein to any specific commercial product, process, or service by trade name, trademark, manufacturer, or otherwise, does not necessarily constitute or imply its endorsement, recommendation, or favoring by the United States Government or any agency thereof. The views and opinions of authors expressed herein do not necessarily state or reflect those of the United States Government or any agency thereof. 


\title{
BLACK LIQUOR GASIFICATION PHASE 2D FINAL REPORT
}

By

\author{
A.L. Kohl \\ A.E. Stewart \\ PREPARED FOR: \\ CHAMPION INTERNATIONAL \\ STAMFORD, CONNECTICUT \\ Work performed under \\ Subcontract STR/DOE-12 \\ of Contract DE-AC05-80CS40341
}

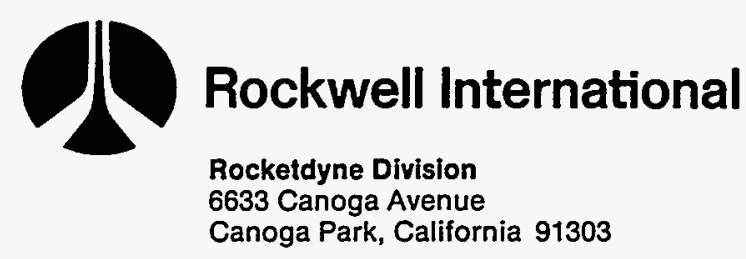


THIS REPORT MAY NOT BE PUBLISHED WITHOUT THE AFPROVAL OF THE DOE OFFICE OF PATENT COUNSEL

\section{DISCLAIMER}

This report was prepared as an account of work sponsored by an agency of the United States Government. Neither the United States Government nor any agency thereof, nor any of their employees, nor any of their contractors, subcontractors, or their employees, makes any warranty, express or implied, or assumes any legal liability or responsibility for the accuracy, completeness, or usefulness of any information, apparatus, product, or process disclosed, or represents that its use would not infringe privately owned rights. Reference herein to any specific commercial product, process, or service by trade name, trademark, manufacturer, or otherwise, does not necessarily constitute or imply its endorsement, recommendation, or favoring by the United States Government or any agency thereof. The views and opinions of authors expressed herein do not necessarily state or reflect those of the United States Government or any agency thereof. 


\section{DISCLAIMER}

Portions of this document may be illegible in electronic image products. Images are produced from the best available original document. 
I. Summary $\ldots \ldots \ldots \ldots \ldots \ldots \ldots \ldots \ldots \ldots \ldots \ldots \ldots \ldots \ldots \ldots \ldots \ldots \ldots \ldots$ I

II. Introduction. $\ldots \ldots \ldots \ldots \ldots \ldots \ldots \ldots \ldots \ldots \ldots \ldots \ldots \ldots \ldots \ldots \ldots \ldots \ldots$

III. Pilot Plant Design.............................

A. Design Objectives and Guidelines.................... 9

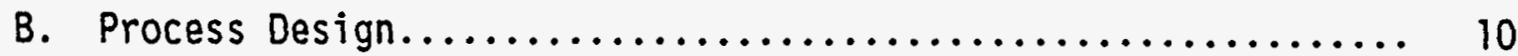

1. Process Description......................... 10

2. Performance Analysis........................ 14

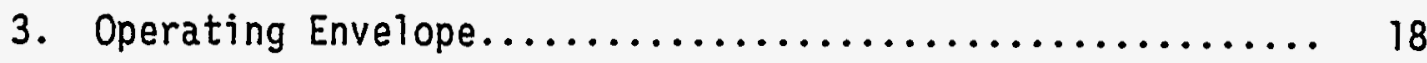

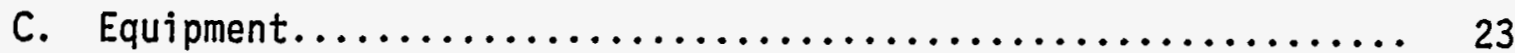

1. Principal Items of Equipment................... 23

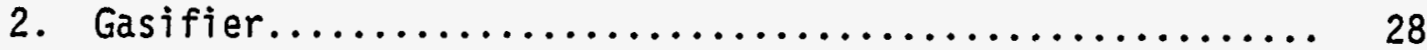

3. Quench System........................... 33

4. Equipment Arrangement...................... 45

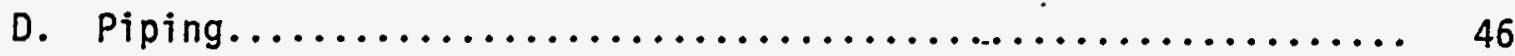

E. Instrumentation and Controls.................... 54

1. Design Guidelines........................... 54

2. Instrumentation and Control System Description.......... 54

IV. Spray Nozzle Testing........................... 71

A. Background and Objectives...................... 71

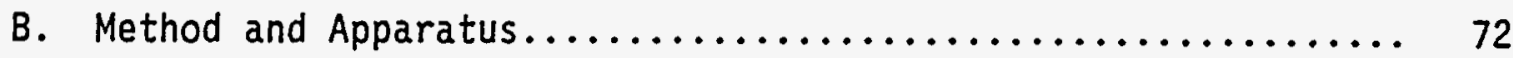

C. Test Results............................... 75

D. Discussion of Results......................... 78

V. Conclusions and Recommendations.................... 87

VI. References.................................. 89 
1. Black Liquor Gasification Pilot Plant Process Flow Diagram....... 11

2. Effect of Black Liquor Feed Rate on Product Gas HHV and Required Air Feed Rate............................. 21

3. Gasifier Operating Envelope........................ 21

4. Quench Tank Operating Envelope....................... 22

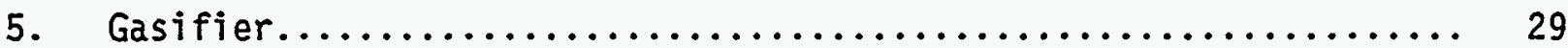

6. Gasifier Refractory Installation.................... 31

7. Product Gas Separator............................ 35

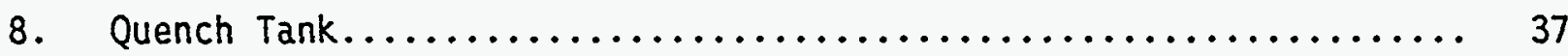

9. Quench Tank Nozzle Details......................... 39

10. Vent Gas Separator............................. 41

11. Corrosion Rate of Carbon Steel in Green Liquor............... 44

12. Black Liquor Gasification Pilot Plant, Plan................ 47

13. Black Liquor Gasification Pilot Plant, Elevations............. 49

14. Schematic Diagram of Spray Nozzle Test Apparatus.............. 73

15. Photographs of Typical Wax Spray Products.................. 81

16. Typical Size Distribution Curves for Spray Products............ 84 


\section{TABLES}

Page

1. Assumptions for Gasifier Performance Calculations.............. 15

2. Calculated Gasifier Performance......................... 17

3. Black Liquor Gasification Pilot Plant Flow Rates for Three Operating Cases................................... 19

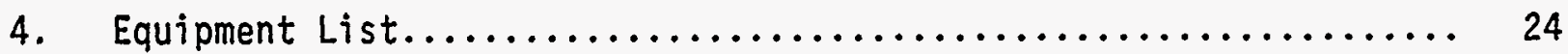

5. Corrosion of Metals in Green Liquor...................... 43

6. Pipeline List..................................... 51

7. Preliminary List of Instrumentation Requirements............. 55

8. Properties of Liquids.................................. 74

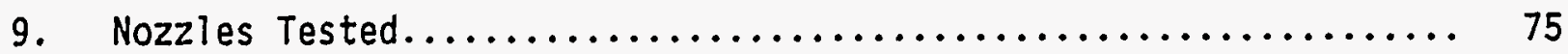

10. Summary of Flow/Pressure Data with Wax................... 76

11. Water Capacity Data................................ 79

12. Size Distribution Results with Hot Wax.................... 80

13. Black Liquor Spray Requirements........................ 82

14. Summary of Hot Wax Spray Test Results..................... 83 


\section{SUMMARY}

This report covers work conducted for Champion International by the Rocketdyne Division of Rockwell International under a subcontract to a Cooperative Agreement between Champion and the Department of Energy (DOE) on the subject of black liquor gasification. The work represents Phase $2 \mathrm{D}$ of a longrange program aimed at developing the Rockwell process for converting concentrated Kraft black liquor into low-Btu fuel gas and reduced melt.

Prior phases have included bench-scale and low-pressure, pilot-scale tests and the conceptual design of a pilot plant capable of operating at pressures up to $45 \mathrm{lb} / \mathrm{in}^{2}$ (absolute). Concurrent with the earlier Rockwell studies, Champion International evaluated the process for application in pulp mills as a possible alternative to the conventional Tomlinson recovery furnace, and concluded that gasification offers many advantages. It was aiso concluded that pilot plant operation at a pressure higher than $45 \mathrm{lb} / \mathrm{in}^{2}$ (absolute) would be desirable to reduce risks in the next step of scaling the process up to a full-scale demonstration unit operating at about $150 \mathrm{lb} / \mathrm{in}^{2}$ (absolute).

A principal objective of the work covered by this report is to upgrade the pilot plant conceptual design to increase its operating pressure and capacity by a factor of approximately 2 (to $90 \mathrm{lb} / \mathrm{in}^{2}$ (absolute) and $2068 \mathrm{lb} / \mathrm{h}$ black liquor feed, respectively). A second objective is to evaluate three types of nozzles previously selected as candidates for feeding black liquor into the gasifier.

The gasifier design was modified to make it capable of $90-1 \mathrm{~b} / \mathrm{in}^{2}$ (absolute) operation, and performance calculations were made for several highpressure cases. At the same time, the computer program for modeling gasifier performance was expanded to include the effect of venting a portion of the hot gas from the gasification zone into the quench tank with the product melt.

AI-DOE-13566 
The calculations showed that venting $10 \%$ of the gas has little effect on product gas quality, but does decrease the temperature at the top of the gasifier by about $68^{\circ} \mathrm{F}$ since less gas is cooled by the black liquor spray. Increasing the operating pressure from 45 to $90 \mathrm{lb} / \mathrm{in}^{2}$ (absolute) while holding the gas velocity constant at $2 \mathrm{ft} / \mathrm{s}$ in the gasification zone doubles the gasifier capacity and causes the predicted heating value of the product gas to increase from 101 to $114 \mathrm{Btu} / \mathrm{scf}$ (dry basis). Increasing the solids concentration of the black liquor from 63 to $75 \%$ at $90-1 \mathrm{~b} / \mathrm{in}^{2}$ (absolute) operating pressure further increases the predicted heating value of the product gas to $118 \mathrm{Btu} / \mathrm{scf}$, but has little effect on black liquor solids gasification capacity.

Material balance calculations were completed showing flow rates and conditions in all major process lines for three reference conditions representing operation at $13.7,45$, and $90 \mathrm{lb} / \mathrm{in}^{2}$ (absolute) in the gasifier. Operating envelopes were developed for the gasifier and quench tanks.

New engineering drawings were made to define the gasifier, gasifier refractory installation, quench tank, product gas separator, vent gas separator, and the equipment arrangement. The equipment list was revised in accordance with design changes and a pipeline list developed giving pipe sizes, schedules, and materials for all major lines.

Design guidelines were developed for the instrumentation and control system and a complete list of instrumentation requirements for the pilot plant was compiled.

A test program was conducted to evaluate three types of nozzles proposed for use in the pilot plant to spray black liquor through the drying zone of the gasifier. The three selected types were a narrow-angle, full-cone pressure nozzle, a two-fluid (pneumatic) nozzle, and a specially designed splashtube nozzle. The tests were conducted by spraying hot molten wax through the nozzles at an elevation of about $11 \mathrm{ft}$ above a catch basin flooded with a horizontal spray of cold water. The wax droplets solidify and are collected 
and characterized by sieve analysis and microscopic examination. The tests indicated that all three types of nozzles could be operated to provide black liquor droplets in the desired size range. The splash-tube and full-cone pressure nozzles are recommended for testing with black liquor in the pilot plant facility. The splash-tube concept is the preferred design primarily because it is less subject to plugging than the full-cone pressure nozzle (which contains internal vanes), and uses much less steam (or air) than the two-fluid nozzle.

It is concluded that the black liquor gasification pilot plant as now configured represents a well-integrated, cost-effective facility for demonstrating all critical operations of the process and providing data for the design of much larger units. Several available spray nozzle designs can be expected to provide effective spraying of black liquor feed in the pilot plant gasifier. One design has been selected that appears to offer both reliable pilot plant performance and scalability to much larger sizes.

$16030 /$ sjh 
AI-DOE- 13566 


\section{INTRODUCTION}

This report covers work conducted by Rockwell International under Amendment 5 to Subcontract STR/DOE-12 of Cooperative Agreement DE-AC-05-80CS40341 between St. Regis Corporation (now Champion International) and the Department of Energy (DOE). The work has been designated Phase 2D of the overall program to differentiate it from prior work under the same subcontract.

The overall program is aimed at demonstrating the feasibility of and providing design data for the Rockwell process for gasifying Kraft black liquor. In this process, concentrated black liquor is converted into low-Btu fuel gas and reduced melt by reaction with air in a specially designed gasification reactor.

Phase 1 of the program was conducted under Subcontract STR/DOE-10 of the St. Regis/DOE cooperative agreement and consisted of nine bench-scale screening tests. This work is described in the Phase 1 final report prepared by St. Regis Corporation. ' Phase 2 of the Rockwell activities initially included 23 bench-scale tests in a 6-in.-diameter gasifier and two runs in a 33-in.-ID pilot-scale unit. This work was extended to include Phase 2A, which consisted of modifying the pilot-scale unit and performing a third extended run.

The bench-scale tests and the three pilot-plant runs are described in detail in Rockwell reports on the subcontract activities 2,3 and in the Champion International Phase 2 final report. ${ }^{4}$

In parallel with the Phase 2 experimental programs conducted by Rockwell, Champion International performed analytical studies to evaluate the black liquor gasification process as a possible alternative to the conventional Tomlinson recovery furnace. They developed detailed computer simulations of overall electric power and steam supply systems for pulp and paper mill 
applications. The study indicated that gasification has high potential and offers many advantages including:

- Higher energy efficiency than state-of-the-art conventional technölogy

- Higher output ratio of electrical power-to-steam, better matching mill demands

- Modularity, making it feasible to add incremental capacity to mills economically

- Significantiy improved safety, eliminating the possibility of smeltwater explosions

- Significantly reduced air emissions

- Reduced capital and operating costs.

The Phase 2 bench-scale tests adequately demonstrated the basic process chemistry and correlated well with theoretical predictions. Product gas higher heating values (HHVs) (dry basis) ranged from about 120 to $140 \mathrm{Btu} / \mathrm{scf}$ depending on black liquor composition and other variables, and sulfur in the melt was generally over $90 \%$ in the form of sodium sulfide.

The three pilot-scale tests demonstrated the operability of the process in engineering-scale equipment and the predictability of gasifier performance. However, the results were adversely affected by a very high heat loss from the pilot-scale gasifier, an existing test unit originally designed for applications requiring a high rate of heat dissipation. The high heat loss resulted in the requirement to operate with a high air/black liquor feed ratio to maintain a constant temperature, and this caused both a low heating value gas and a poorly reduced melt to be produced.

It was concluded that additional pilot-plant tests would be desirable to provide an engineering-scale demonstration of the process, including the production of gas with a reasonably high heating value (over about 90 Btu/scf), the production of highly reduced melt, operation at elevated pressure, and melt discharge and quenching in a closed system. 
The next two phases of the program (Phases $2 \mathrm{~B}$ and $2 \mathrm{C}$ ) were aimed at developing the conceptual design of a pilot-scale gasification plant that would overcome the limitations of the existing facility and accomplish the above objectives. They also included specific tasks to evaluate refractory materials for use in the gasifier; develop the conceptual design of a melt discharge and quenching system for use in a pressurized environment; and study the requirements and potential techniques for spraying black liquor into the gasifier. The results of these activities are described in the Rockwell Phase $2 B$ and $2 C$ final reports ${ }^{5,6}$ and in a comprehensive report by Dr. E. Kelleher of Champion International. ${ }^{7}$

The pilot plant design resulting from the above studies was based on an operating pressure of $45 \mathrm{lb} / \mathrm{in}^{2}$ (absolute), representing a factor of 3 increase over the previous atmospheric pressure tests. Subsequent to this design work, it was concluded that higher pressure pilot plant operation would be desirable to further reduce risks in scaling the process up to a demonstration plant operating at about $150 \mathrm{lb} / \mathrm{in}^{2}$ (absolute).

A preliminary review of the pilot plant design and the capabilities of existing facilities was made to determine the maximum operating pressure at which meaningful tests could be conducted without a major increase in the cost and scope of the pilot plant program. Since the gasifier throughput is limited by gas velocity to avoid excessive entrainment, the design black liquor feed rate for a given size gasifier increases directly with pressure. The quantity of black liquor to be handled was, therefore, one factor in determining the optimum design pressure. Other factors were the flow and pressure capabilities of the existing air compressors and air heaters and the capacity of the existing product gas combustor. It was concluded that valuable high-pressure performance data could be obtained by operating the pilot plant for relatively short periods at a pressure of about $90 \mathrm{lb} / \mathrm{in}^{2}$ (absolute) in conjunction with longer runs at lower pressure and feed rate conditions, and that this change would not require a major increase in scope of the pilot plant program. 
A principal objective of this phase of the program (2D) is to upgrade the pilot plant conceptual design to increase both its operating pressure and throughput by a factor of approximately 2. A second objective is to evaluate spray nozzles proposed for feeding black liquor into the gasifier by conducting spray tests using molten wax to determine spray patterns and drop sizes.

$1603 \mathrm{D} / \mathrm{sjh}$

AI-DOE-13566 


\section{PILOT PLANT DESIGN}

A. DESIGN OBJECTIVES .AND GUIDELINES

The specific objective of the pilot plant design is to provide a facility that will demonstrate satisfactory operation and performance of the black liquor gasification process at pressures up to $90 \mathrm{ib} / \mathrm{in}^{2}$ (absolute) and black liquor feed rates up to about $2000 \mathrm{lb} / \mathrm{h}$, including the production of gas with a higher heating value (HHV) of about $100 \mathrm{Btu} / \mathrm{scf}$ (dry basis) and the production of melt in which over $90 \%$ of the sulfate is reduced to sulfide.

Additional objectives are to provide the capabilities for: (1) determining the effects of gasifier temperature, pressure, feed rate, air distribution pattern, melt pool depth, and black liquor composition; (2) evaluating the performance of components and materials; and (3) procuring data for the design of larger plants.

A key requirement of the design is that the new gasifier be compatible with existing auxiliary facilities but have the low heat loss and highpressure capabilities necessary for it to meet the specific objectives given above. Existing test facilities that will be incorporated into the overall pilot-plant system include the feed air compressors, feed air heater, product gas baghouse, product gas combustor, data acquisition and control center (DAC), and gas analysis instrumentation.

The plant must meet all safety and environmental requirements at the site; however, it may be designed for a relatively short operating life ( $\sim 2$ years). It is expected that this criterion will permit the use of lowcost construction materials such as carbon steel in the green liquor circuit and in other moderately corrosive environments of the pilot-plant system. 
The following specific design criteria have been established for the pilot plant:

- Internal gasifier dimensions:

-30-in. internal diameter by

17-ft active height

- Operating pressure capability:

$901 \mathrm{~b} / \mathrm{in}^{2}$ (absolute) $(\sim 6 \mathrm{~atm})$

- Gas velocity at maximum pressure:

$\sim 2 \mathrm{ft} / \mathrm{s}$

- Feed air temperature:

$\sim 700^{\circ} \mathrm{F}$

- Black liquor feed rate capability: $2068 \mathrm{lb} / \mathrm{h}$

- Black liquor solids concentration: $-63 \%$

- Black liquor feed temperature:

$220^{\circ} \mathrm{F}$

- Pool depth (maximum):

24 in.

- Air injection levels:

10 and 32 in.

- Black liquor spray nozzles:

four feed points

- Heat loss from gasification zone:

$<600 \mathrm{Btu} / 1 \mathrm{~b}$ of feed

- Melt quench medium:

green liquor

- Melt shatter jets:

steam and recycled green liquor

- Product gas heating value:

-100-Btu/scf HHV, dry basis, at maximum throughput.

\section{B. PROCESS DESIGN}

\section{Process Description}

A flowsheet of the overall pilot-plant gasification system is given in Figure 1. Concentrated black liquor will be delivered to the site by tank truck and pumped into black liquor tank $V-5$ for storage. This tank is equipped with a heater to maintain the temperature of the black liquor above approximately $200^{\circ} \mathrm{F}$, an agitator to minimize settling or stratification, and a charcoal vent filter to prevent the release of odorous gases to the atmosphere. During operation of the system, a high volume of black liquor is 

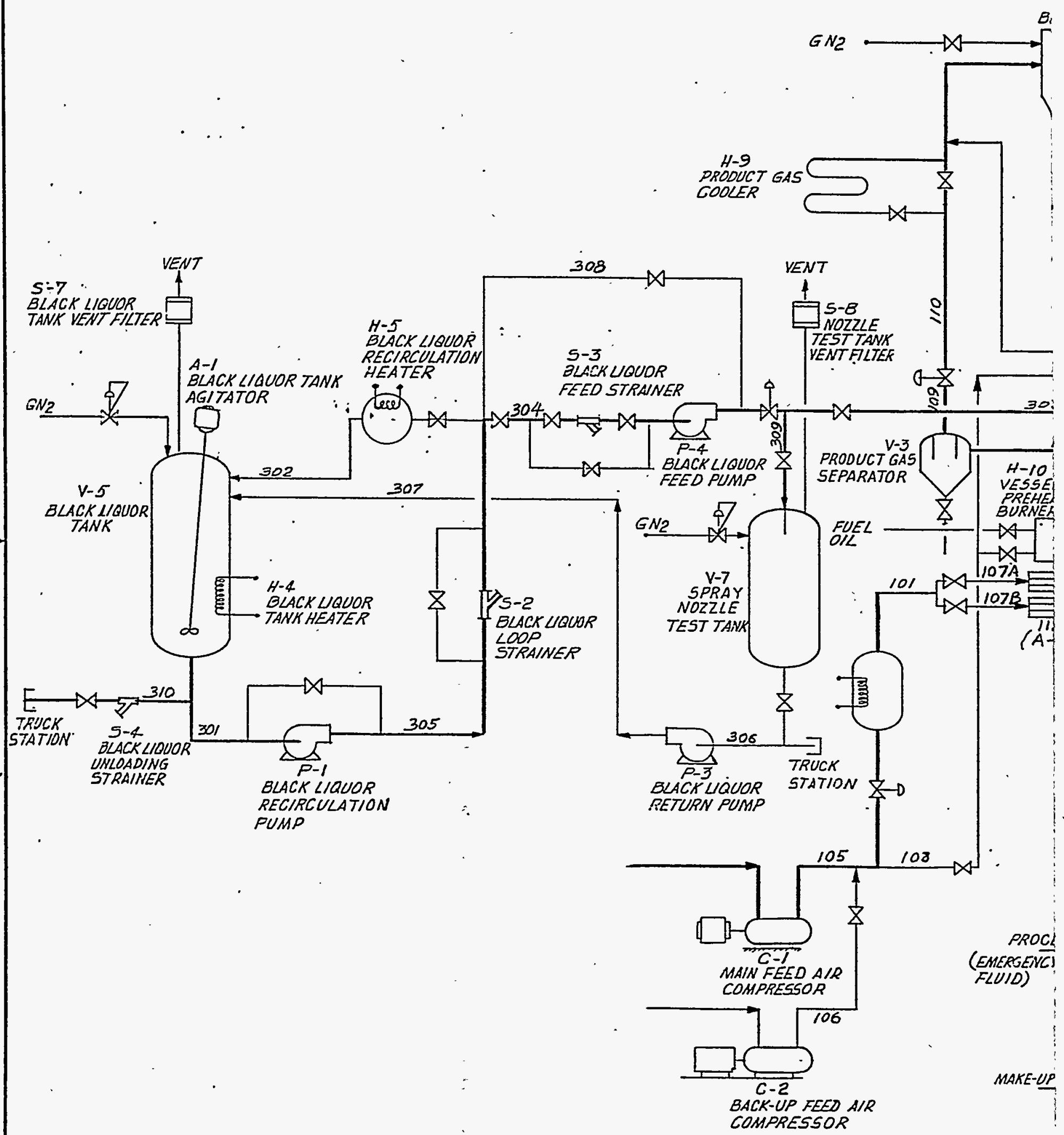
continuously pumped out of the black liquor tank by pump P-1 through a full stream strainer and the black liquor recirculation heater $\mathrm{H}-5$ and returned to the tank. This recycle system serves to provide a clean, well-mixed, and heated source of black liquor close to the gasifier vessel to supply the smallvolume gasifier feed stream.

The gasifier feed stream passes through a fine-screen strainer and a highpressure pump $(P-4)$ before being injected into the top of the gasifier $(V-1)$ through one to four spray nozzles. In the gasifier, the droplets of black liquor fall downward through a drying zone countercurrent to a rising stream of hot product gas. Gasification occurs at the bottom of the gasifier, where heated air reacts with the carbonaceous material in the black liquor under substoichiometric (partial oxidation) conditions. The partial oxidation reactions are exothermic and maintain the temperature in the gasification zone at about $1750^{\circ} \mathrm{F}$. Inorganic salts in the black liquor melt and form a pool at the bottom of the gasifier, the depth of which is determined by the location of an overflow port.

Feed air for the gasifier is compressed in compressors $C-1$ and $C-2$ and heated to about $700^{\circ} \mathrm{F}$ in feed air heater $\mathrm{H}-2$ before it enters the gasifier. $\mathrm{A}$ portion of the air is fed above the surface of the pool of melt to promote gasification reactions in this region, and the balance is fed directly into the melt pool to ensure that char entering the melt is oxidized.

After steady-state operation is attained, product melt continuously over- 1 flows from the pool and, together with a portion of the hot gas, flows to the quench tank $(V-2)$. The melt is shattered by jets of steam and recycled green liquor as it falls into the pool of green liquor in the quench tank. The green liquor is maintained at a temperature of about $220^{\circ} \mathrm{F}$ when the system is at elevated pressure (or $180^{\circ} \mathrm{F}$ at atmospheric pressure) by the use of a heater $(\mathrm{H}-6)$ or cooler $(\mathrm{H}-8)$ in the green liquor recirculation line. Green liquor is removed from the quench tank through a strainer, which retains large 
undissolved particles of solidified melt, and a high volume pump $(P-2)$. Most of the pump product is recirculated through the shatter jet nozzle, but a small amount, representing the net product, flows through a finer strainer to the green liquor storage tanks $(V-6 A$ and $B)$.

A small fraction of the gas generated in the gasification zone is allowed to flow out of the gasifier with the melt to aid in maintaining a sufficientiy high temperature in the melt discharge line. A gas burner, $\mathrm{H}-3$, is also provided in this line as an auxiliary heat source. Gas which enters the quench tank with the melt stream is vented through a separator and joins the product gas stream after pressure reduction. The main stream of product gas, which is cooled from approximately $1750^{\circ} \mathrm{F}$ to less than $1000^{\circ} \mathrm{F}$ in the drying zone of the gasifier, leaves the top of the gasifier and passes through a cyclone separator to remove relatively large solid particles then through a pressure control valve, which reduces its pressure to near atmospheric.

After pressure reduction the product gas flows through a cooler, $\mathrm{H}-9$, which reduces its temperature to less than $500^{\circ} \mathrm{F}$. It then enters the baghouse, S-1, which removes very fine solid particles, primarily sodium carbonate fume. The cleaned product gas is finally burned with excess air in combustor, $\mathrm{H}-\mathrm{T}$, and the resulting flue gas is vented to the atmosphere.

\section{Performance Analys is}

The basic computer program used for the gasifier performance calculations was described in a previous report. ${ }^{2}$ During, the current phase of the program, the calculation procedure has been upgraded to incorporate the effects of venting about $10 \%$ of the high-temperature gas stream through the melt discharge line. The purpose of the vent gas is to aid in maintaining an elevated temperature over the flowing stream of melt.

Key assumptions used in the performance calculations are listed in Table 1 .

AI-DOE-13566 
Table 1. Assumptions for Gàsifier Performance Calculations

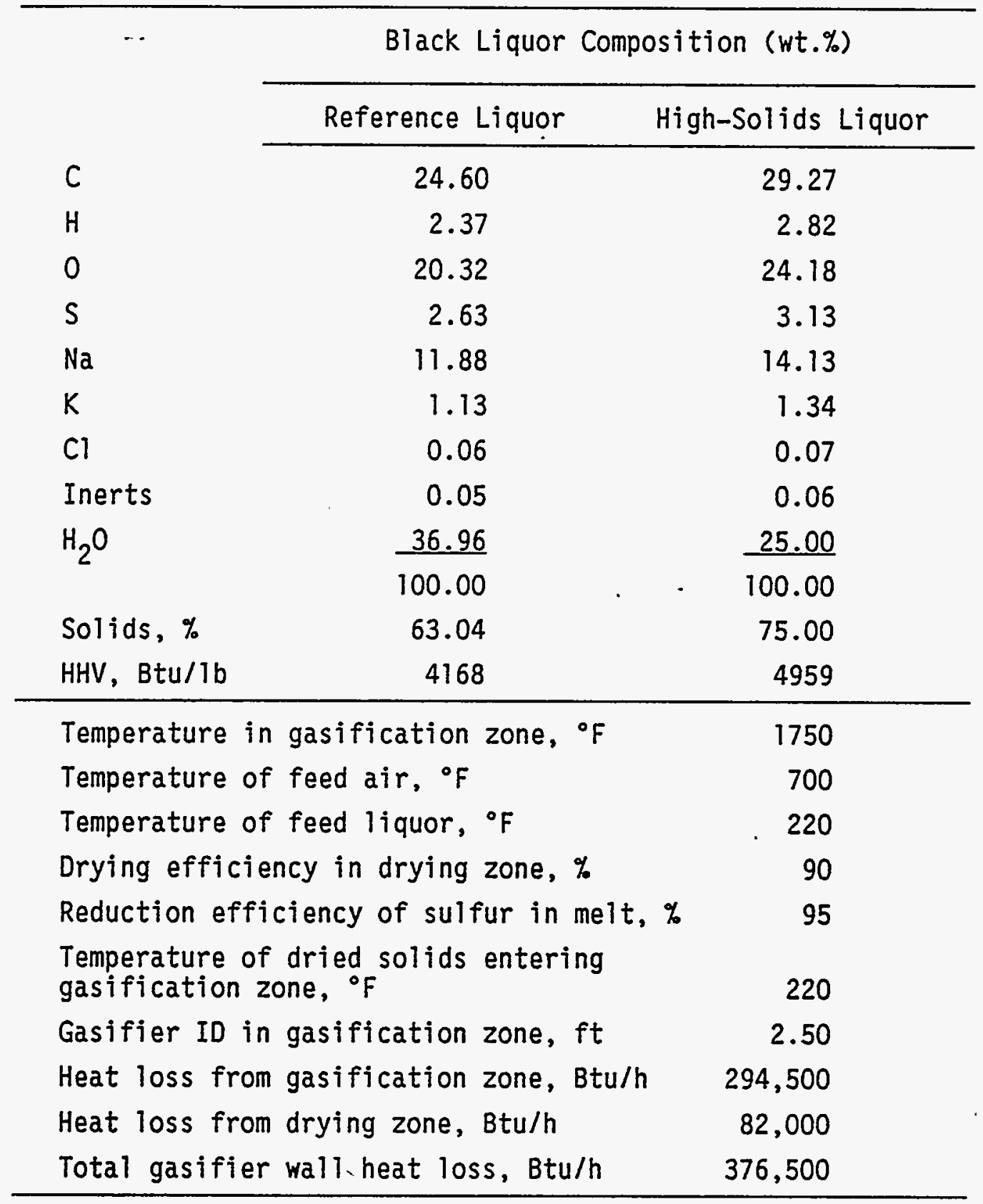

$1603 \mathrm{D} / \mathrm{rmr}$ 
Several additional assumptions are required to calculate the cases with partial gas venting. It is assumed that $10 \%$ of the total gas volume leaving the gasification zone of the gasifier is allowed to flow through the melt discharge line and the remaining 90\% flows upward through the drying zone. The gas leaving the gasification zone is assumed to be in equilibrium with regard to the water gas shift reaction at $1750^{\circ} \mathrm{F}$, and the $10 \%$ vented gas, therefore, has a composition corresponding to this equilibrium. The $90 \%$ stream picks up additional water that is evaporated from the black liquor in the drying zone of the gasifier. For calculation purposes, this stream is assumed to reequilibrate with the evaporated water at a temperature of $1750^{\circ} \mathrm{F}$. Gas compositions measured during previous tests agree better with published equilibrium data when the gasification zone temperature rather than a lower drying zone temperature is assumed.

The results of gasifier performance calculations for seven typical cases are given in Table 2 .

Cases $A, B$, and $C$ represent the reference design conditions for operation at nominal pressures of 7,3 , and 6 atmospheres, respectively. The reference design cases are based on a superficial gas velocity in the gasifier of $3.0 \mathrm{ft} / \mathrm{s}$ when the gasifier is operated at one atm $\left(13.7 \mathrm{lb} / \mathrm{in}^{2}\right.$ (absolute) at the test site) and $2.0 \mathrm{ft} / \mathrm{s}$ when the nominal pressure is 3 or $6 \mathrm{~atm}$. These gas velocities are considered conservative and are based primarily on consideration of entrainment of black liquor and melt droplets in the up-flowing gas stream. Case $D$ is included to show the effect of increasing the allowable gas velocity from 2.0 to $2.5 \mathrm{ft} / \mathrm{s}$ at $901 \mathrm{~b} / \mathrm{in}^{2}$ (absolute). This results in a $26 \%$ increase in feed capacity and a slight increase in product gas heating value.

Case $E$ provides an indication of the effect of black liquor concentration on performance. For this case it is assumed that the black liquor has the very high solids concentration of $75 \%$ (versus $63 \%$ for the other cases). Comparing Case $E$ with reference Case $C$ shows that the increase in solids concentration reduces the black liquor feed capacity by $17 \%$ (but increases the 
Table 2. Calculated Gasifier Performance

\begin{tabular}{|c|c|c|c|c|c|c|c|}
\hline \multirow[b]{2}{*}{$\cdots$} & \multicolumn{6}{|c|}{ Pilot Plant Cases } & \multirow{2}{*}{$\begin{array}{c}\text { Commercial } \\
\text { Plant } \\
\text { Design }\end{array}$} \\
\hline & A & $\mathbf{8}$ & C & D & E & $F$ & \\
\hline Pressure, $1 \mathrm{~b} / \mathrm{in}^{2}$ (absolute) & 13.7 & 45.0 & 90.0 & 90.0 & 90.0 & 90.0 & 233 \\
\hline Sotids, \% & 63.0 & 63.0 & 63.0 & 63.0 & 75.0 & 63.0 & 63.0 \\
\hline Black Tiquor feed rate, $1 \mathrm{~b} / \mathrm{h}$ & 391 & 982 & 2068 & 2610 & 1776 & 2068 & 65,540 \\
\hline Gas velocity, $\mathrm{ft} / \mathrm{s}^{\star}$ & 3.00 & 2.00 & 2.00 & 2.50 & 2.00 & 2.00 & 2.00 \\
\hline Air, \% of theoretical & 56.4 & 45.2 & 41.4 & 40.7 & 40.8 & 41.4 & 38.9 \\
\hline $\begin{array}{l}\text { Gas vented to quench tank, } \% \\
\text { Material balance } 1 b / 1 b \text { of black liquor }\end{array}$ & 10 & 10 & 10 & 10 & 10 & 0 & 0 \\
\hline Air & 1.76 & 1.41 & 1.29 & 1.27 & 1.52 & 1.29 & 1.22 \\
\hline Gasifier outlet gas & 2.27 & 1.95 & 1.85 & 1.83 & 1.99 & 2.01 & 1.94 \\
\hline Vent gas & 0.21 & 0.78 & 0.17 & 0.17 & 0.20 & - & - \\
\hline Melt & 0.28 & 0.28 & 0.28 & 0.28 & 0.33 & 0.28 & 0.28 \\
\hline Gasifier outlet gas & & & & & & & \\
\hline Volume, scf/lb of blach liquor & 33.8 & 30.3 & 29.2 & 29.0 & 30.5 & 31.6 & 30.8 \\
\hline Temperature, of & 796 & 884 & 921 & 928 & 1200 & 989 & 1024 \\
\hline Water content, $\%$ & 25.4 & 24.9 & 24.7 & 24.6 & 16.8 & 23.2 & 22.9 \\
\hline $\begin{array}{l}\text { HHV, Btu/scf (dry) } \\
\text { Composition, \% (dry basis) }\end{array}$ & 70 & 101 & 113 & 115 & 117 & 113 & 121 \\
\hline $\mathrm{CO}_{2}$ & 16.4 & 15.4 & 15.0 & 14.9 & 12.9 & 14.7 & 14.4 \\
\hline $\mathrm{CO}^{\circ}$. & 7.2 & 10.3 & 17.5 & 11.8 & 14.4 & 12.0 & 12.9 \\
\hline $\mathrm{CH}_{4}$ & 1.1 & 1.7 & 1.8 & 1.9 & 1.9 & 1.8 & 1.9 \\
\hline $\mathrm{C}_{2} \overrightarrow{\mathrm{H}}_{4}$ & 0.15 & 0.17 & 0.18 & 0.18 & 0.18 & 0.18 & 0.18 \\
\hline $\mathrm{H}_{2}^{2}$ & 9.8 & 14.5 & 16.4 & 16.8 & 14.8 & 16.1 & 17.4 \\
\hline $\mathrm{H}_{2} \mathrm{~S}$ & 0.14 & 0.16 & 0.17 & 0.17 & 0.17 & 0.17 & 0.17 \\
\hline $\mathrm{N}_{2}$ & 64.2 & 57.8 & 54.9 & 54.3 & 55.7 & 55.1 & 53.1 \\
\hline Vent gas & & & & & & & \\
\hline Volume, scf/lb of black liquor & 3.0 & 2.6 & 2.5 & 2.4 & 2.9 & & \\
\hline Temperature, ${ }^{\circ} \mathrm{F}$ & 1750 & 1750 & 1750 & 1750 & 1750 & & \\
\hline Water content, $\%$ & 9.5 & 7.9 & 7.2 & 7.1 & 6.3 & & \\
\hline $\begin{array}{l}\text { HHV, Btu/scf (dry) } \\
\text { Composition, \% (dry basis) }\end{array}$ & 72 & 106 & 120 & 123 & 123 & & \\
\hline $\mathrm{CO}_{2}$ & 12.0 & 10.3 & 9.2 & 9.0 & 8.5 & & \\
\hline $\mathrm{CO}^{2}$ & 11.6 & 17.0 & 19.2 & 19.6 & 20.2 & & \\
\hline $\mathrm{CH}_{4}$ & 1.2 & 1.8 & 2.0 & 2.0 & 2.0 & & \\
\hline $\mathrm{C}_{2} \mathrm{H}_{4}$ & 0.16 & 0.18 & 0.19 & 0.19 & 0.19 & & \\
\hline $\mathrm{H}_{2}$ & 6.1 & 9.3 & 10.6 & 10.9 & 10.5 & & · \\
\hline $\mathrm{H}_{2}^{2} \mathrm{~S}$ & 0.15 & 0.17 & 0.18 & 0.18 & 0.18 & & \\
\hline $\mathrm{N}_{2}$ & 67.8 & 61.3 & 58.6 & 58.1 & 58.5 & & \\
\hline Total product gas & & & & & & & \\
\hline Volume, (dry) scf/lb of black liquor & 27.9 & 25.2 & 24.3 & 24.1 & 28.1 & 24.3 & 23.8 \\
\hline HHV, Btu/scf (dry) & 70 & 101 & 114 & 116 & 118 & 113 & 121 \\
\hline
\end{tabular}

*Based on cross-sectional area of the gasification zone.

$16030 / \mathrm{rmr}$ 
black liquor solids feed capacity by $2 \%$ ) and increases the product gas heating value by about $3.5 \%$. Its main effect is on the temperature of the product gas which increases from 921 to 1200 because of the much smaller amount of water evaporated in the drying zone.

Case $F$ is identical to reference Case $C$ except that no gas is vented through the quench tank. The product gas quantity and heating value are not changed significantly; however, the gasifier outlet gas discharged from the top of the gasifier is about $68^{\circ} \mathrm{F}$ hotter because there is about $10 \%$ more to be cooled by the black liquor spray.

A commercial plant design case is included for comparison with the projected pilot plant results. This case does not include the venting of part of the product gas through the quench tank and, therefore, can best be compared with pilot plant Case $F$. The commercial plant is projected to produce slightly less gas/lb of black liquor feed, but the gas will have a higher heating value (121. Btu/scf versus $113 \mathrm{Btu} / \mathrm{scf})$. This effect is due entirely to the lower relative heat loss of the commercial size plant.

Flow rates, stream characteristics, temperatures, and pressures in key process lines are given in Table 3 for Cases $A, B$, and $C$. These cases represent: $A$ - operation at $13.7 \mathrm{lb} / \mathrm{in}^{2}$ (absolute) and $3 \mathrm{ft} / \mathrm{s}$ superficial gas velocity, B - operation at $45 \mathrm{lb} / \mathrm{in}^{2}$ (absolute) and $2 \mathrm{ft} / \mathrm{s}$, and $\mathrm{C}$-operation at $90.0 \mathrm{lb} / \mathrm{in}^{2}$ (absolute) and $2 \mathrm{ft} / \mathrm{s}$. Case $C$ represents the reference design conditions for the gasifier; however, the other cases are included as they may impose special requirements on the pilot plant systems.

\section{Operating Envelope}

Figures 2, 3, and 4 define the normal operating envelopes and predicted performance for the pilot plant. The figures are based on the assumptions for gasifier performance calculations given in Table 2. The points marked $A, B$, and $C$ on the charts represent the three cases described above. 


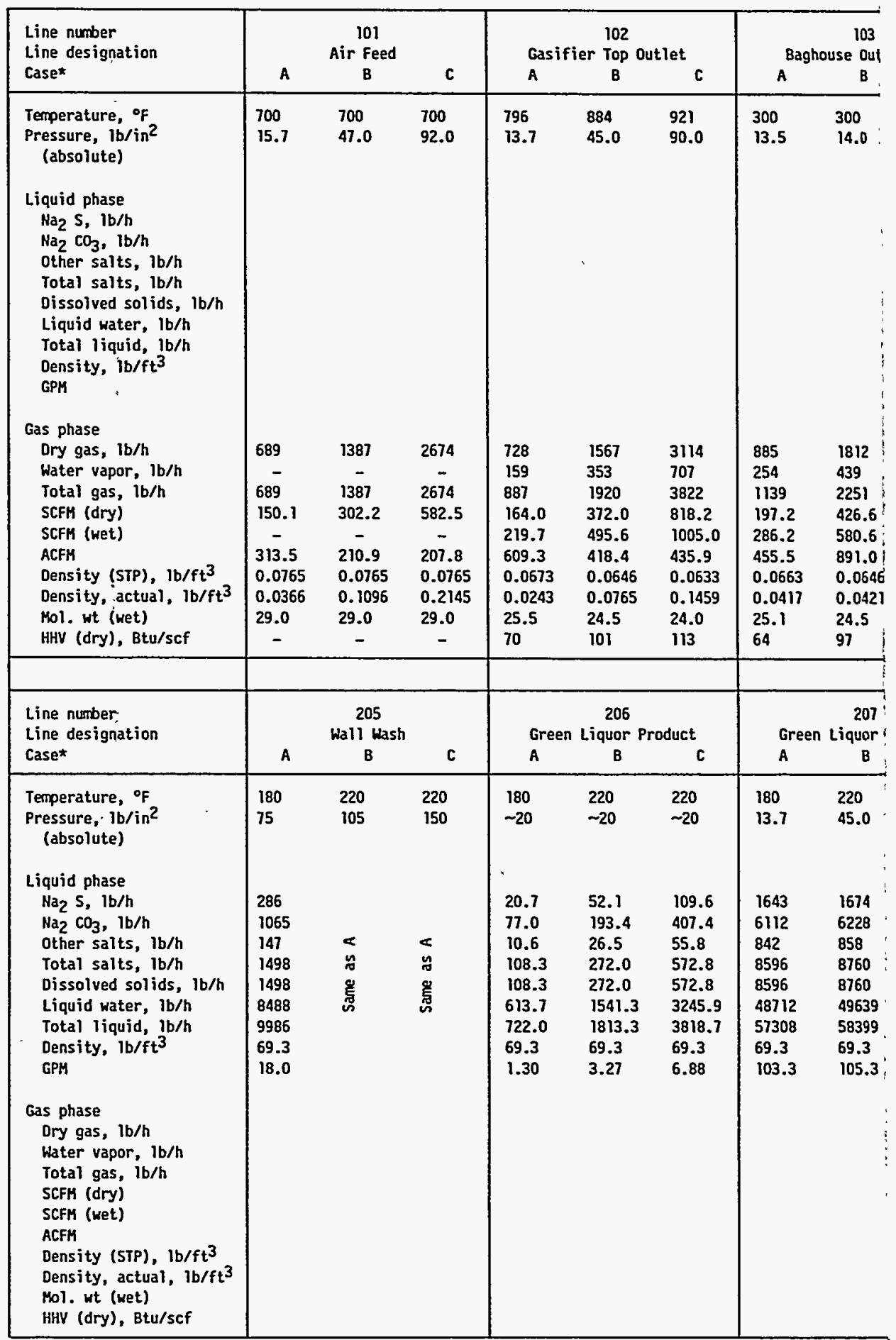

*Key operating conditions:

A - Atmospheric pressure operation, superficial velocity $3.0 \mathrm{ft} / \mathrm{s}$ in gasifier, $391 \mathrm{lb} / \mathrm{h} \mathrm{B.L}$. feed $B-45 \mathrm{lb} / \mathrm{in}^{2}$ (absolute) operation, superficial velocity $2.0 \mathrm{ft} / \mathrm{s}$ in gasifier, $982 \mathrm{lb} / \mathrm{h} \mathrm{B.L}$. feed $\mathrm{C}-90 \mathrm{lb} / \mathrm{in}^{2}$ (absolute) operation, superficial velocity $2.0 \mathrm{ft} / \mathrm{s}$ in gasifier, $2,068 \mathrm{lb} / \mathrm{h} \mathrm{B.L}$. feed 
Table 3. Black Liquor Gasification

Pilot Plant Flow Rates for

Three Operating Cases

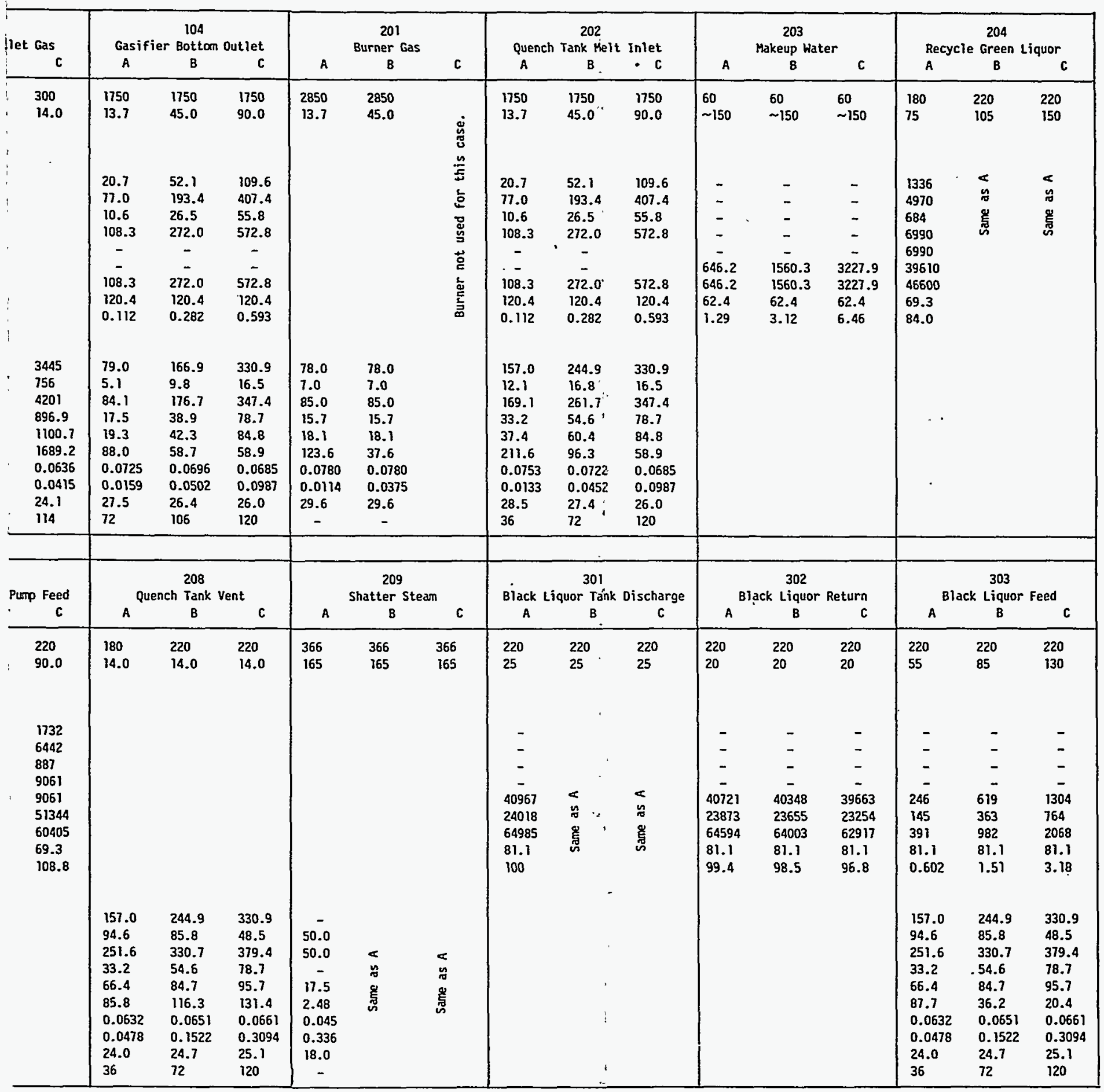




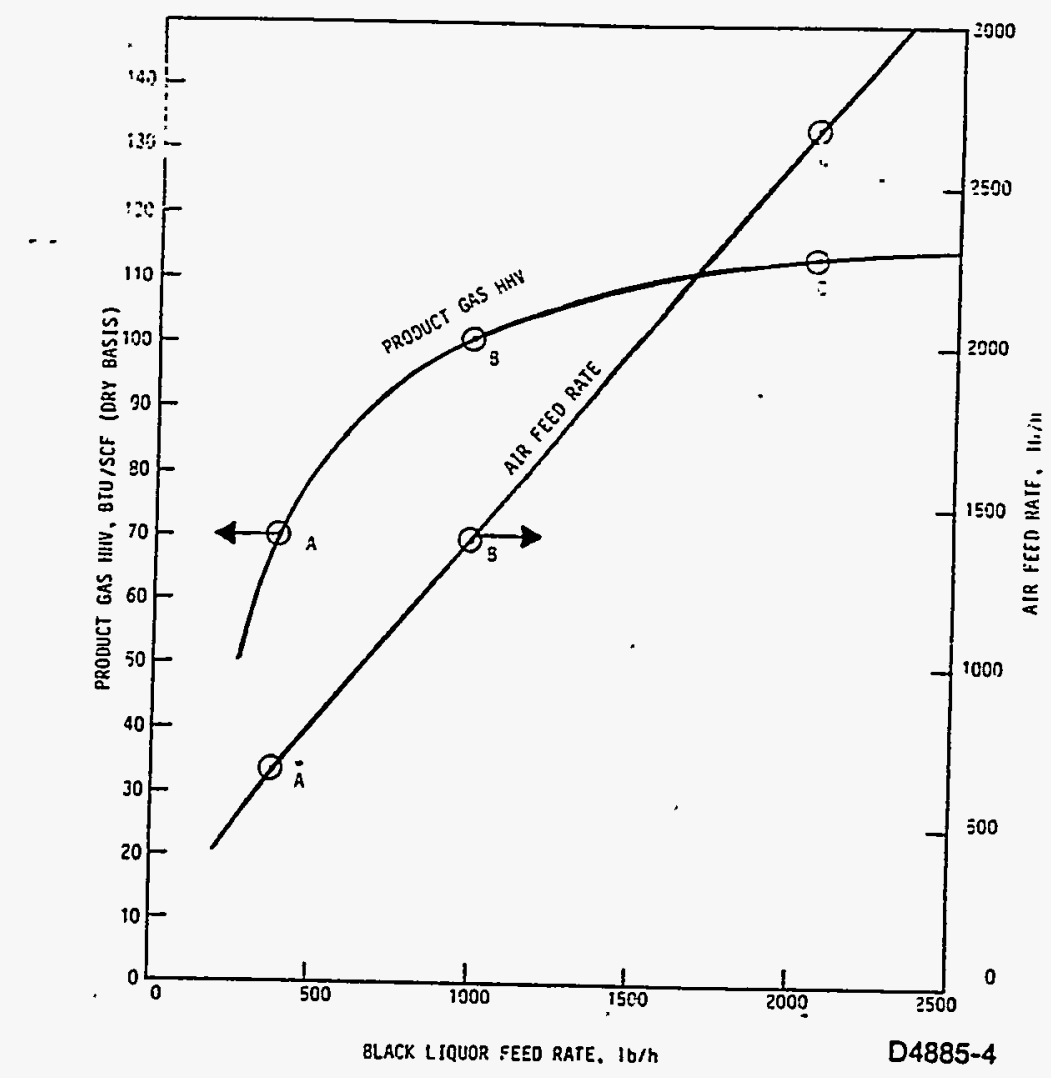

Figure 2. Effect of Black Liquor Feed Rate on Product Gas HHV and Required Air Feed Rate

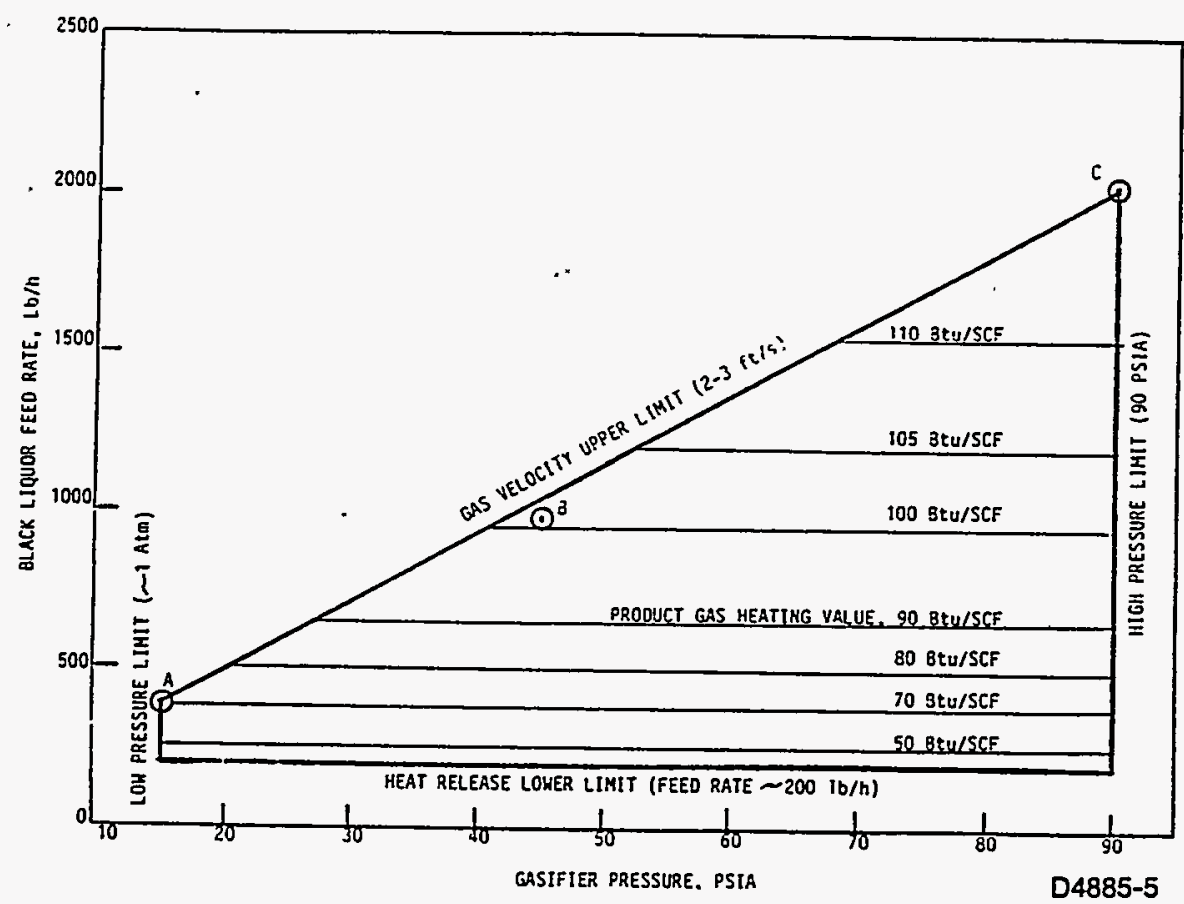

Figure 3. Gasifier Operating Envelope

$$
\text { AI-DOE-13566 }
$$




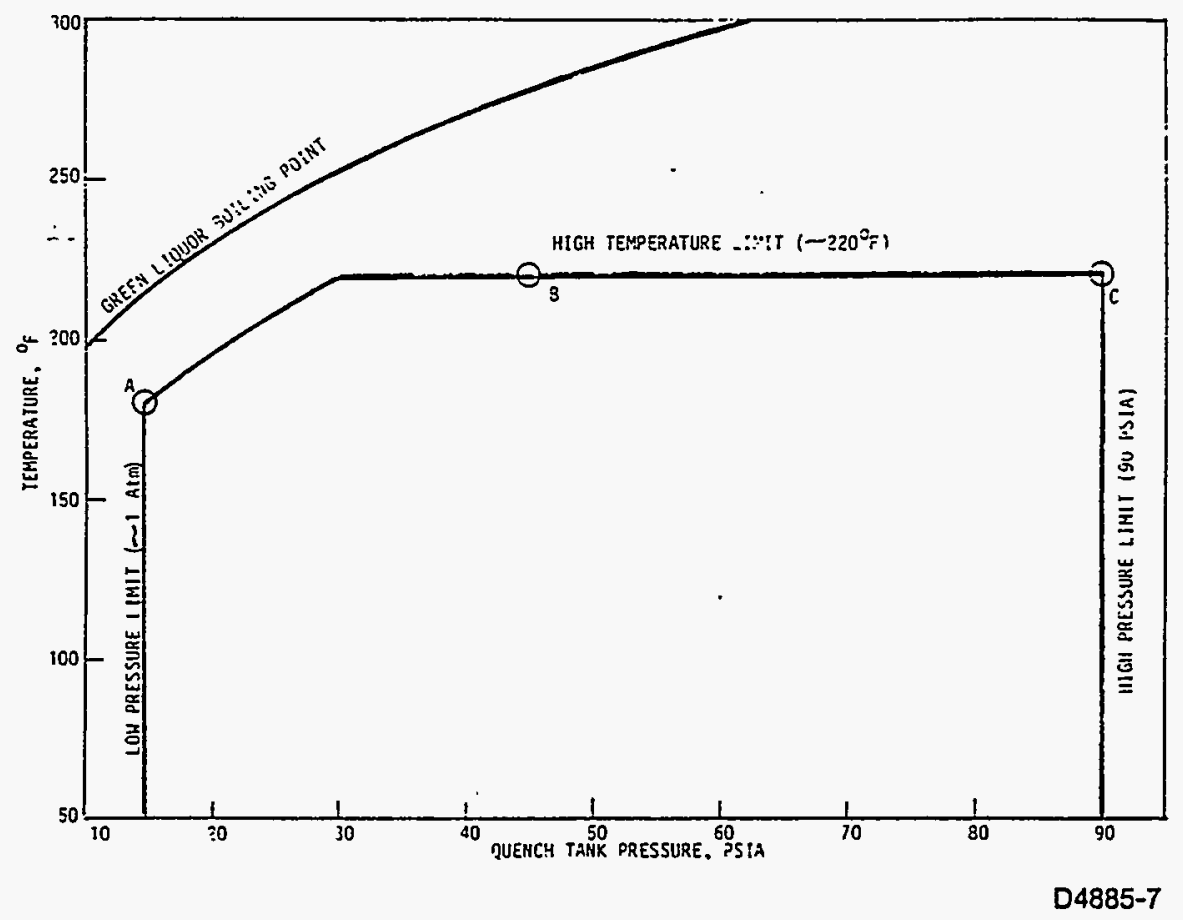

Figure 4. Quench Tank Operating Envelope

Figures 2 and 3 are intended as guides to gasifier operation. Figure 2 indicates the calculated air rate required for steady-state operation at any given black liquor feed rate and the predicted product gas HHV. The HHV Values are for the gas as it leaves the gasifier prior to mixing with vent gas from the quench tank. The air feed rate is also based on the gasifier alone and does not include air required to operate the burner in the melt discharge line. The chart indicates that it should be possible to produce gas with a heating value over $100 \mathrm{Btu} / \mathrm{scf}$ by operating with a black liquor feed rate higher than about $1000 \mathrm{lb} / \mathrm{h}$, but the product gas heating value falls off very rapidly if the black liquor feed rate is reduced below about $500 \mathrm{lb} / \mathrm{h}$.

Figure 3 represents the gasifier operating envelope. Operation anywhere within the indicated boundaries is acceptable and can be expected to result in a product gas with the heating value given on the horizontal lines. The boundaries represent normal operating criteria and not physical limitations of the equipment. The gas velocity upper limit of $2 \mathrm{ft} / \mathrm{s}$ at $90 \mathrm{lb} / \mathrm{in}^{2}$ (absolute) or $3 \mathrm{ft} / \mathrm{s}$ at atmospheric pressure is quite conservative and may be exceeded 
for special tests. Similarly, the high-pressure limit of $901 \mathrm{~b} / \mathrm{in}^{2}$ (absolute) $\left(\sim 76 \mathrm{lb} / \mathrm{in}^{2}\right.$ [gage] at the test site) is well below the pressure rating of the gasifier/quench system (150 1b/in ${ }^{2}$ [gage]) so higher pressure operation is a possibility. However, it is believed that all test objectives can be met by successfully operating the pilot plant within the indicated envelope.

Figure 4 shows the operating envelope for the quench tank. A maximum temperature of $220^{\circ} \mathrm{F}$ has been selected to minimize the corrosion of carbon steel. Since this temperature is above the boiling point of green liquor at atmospheric pressure $\left(\sim 215^{\circ} \mathrm{F}\right)$, the high-temperature limit is reduced to $180^{\circ} \mathrm{F}$ at atmospheric pressure. It is expected that some boiling will occur where the melt enters the pool of green liquor, but the $35^{\circ} \mathrm{F}$ subcooling of the bulk of the solution will prevent excessive steam evolution and release with the vent gas.

C. EQUIPMENT

1. Principal Items of Equipment

A listing and brief description of all major process equipment items identified on the Process Flow Diagram (Figure 1) is given in Table 4. The list also indicates the proposed source of the items as follows:

1. Special equipment to be designed and fabricated. This category includes the gasifier, quench tank with melt discharge line and other accessories, product gas and vent gas separators, and the product gas cooler, which will be fabricated from standard pipe. Conceptual design drawings have been prepared on these equipment items.

2. Standard equipment to be purchased. This category includes relatively standard items such as pumps, strainers, heat exchangers, burners, and agitators, which will require procurement for the pilot plant. This category also includes two lowpressure tanks, the black liquor tank, and spray nozzle test tank, which will require some design work before procurement.

3. Existing Rockwell equipment. This category consists primarily of those portions of the existing molten salt test facility which will be incorporated into the pilot plant. The key items

$$
\text { AI-DOE-13566 }
$$


Table 4. Equipment List (Sheet 1 of 4 )

\begin{tabular}{|c|c|c|c|}
\hline \multicolumn{2}{|r|}{ Item } & Description & Source* \\
\hline \multicolumn{4}{|c|}{ Vessels and Tanks } \\
\hline$V-1$ & Gasifier & $\begin{array}{l}\text { Vertical cylindrical tank, } 5 \mathrm{ft} \\
7-1 / 2 \text { in. OD } \times 24 \mathrm{ft} 6 \text { in. total } \\
\text { height. Carbon steel shell, refrac- } \\
\text { tory lining, design conditions } \\
1501 \mathrm{~b} / \mathrm{in}^{2} \text { (gage), } 650^{\circ} \mathrm{F} \text { (shell) }\end{array}$ & 1 \\
\hline$V-2$ & Quench tank & $\begin{array}{l}\text { Horizontal cylindrical tank, } 5 \mathrm{ft} \\
6 \text { in. OD } x 11 \mathrm{ft} 6 \text { in. total } \\
\text { length with vertical extension } \\
4 \mathrm{ft} \text { OD } x \sim 8 \text { ft height. Carbon } \\
\text { steel, design conditions } 150 \mathrm{lb} / \mathrm{in}^{2} \\
\text { (gage), } 650^{\circ} \mathrm{F}\end{array}$ & 1 \\
\hline$V-3$ & Product gas separator & $\begin{array}{l}\text { Cyclonic separator, } 24 \text { in. OD } x \\
\sim 86 \text { in. total height, } 304 \text { stain- } \\
\text { less steel, design conditions } \\
1501 \mathrm{~b} / \text { lin }^{2} \text { (gage), } 650^{\circ} \mathrm{F}\end{array}$ & 1 \\
\hline$V-4$ & Vent gas separator & $\begin{array}{l}\text { Cyclonic separator, } 12 \text { in. Sched- } \\
\text { ule } 40 \text { pipe body. } 48 \text { in. total } \\
\text { length. Carbon steel design } \\
\text { conditions } 150 \text { lb/in' (gage), } \\
650^{\circ} \mathrm{F}\end{array}$ & 1 \\
\hline$V-5$ & Black liquor tank & $\begin{array}{l}9000 \mathrm{gal}, 10 \mathrm{ft} \text { diam } \times 10 \mathrm{ft} \text { high, } \\
\text { carbon steel, design conditions: } \\
50 \mathrm{lb} / \mathrm{in}^{2} \text { (gage), } 250^{\circ} \mathrm{F}\end{array}$ & 2 \\
\hline $\begin{array}{l}V-6 \\
A \& B\end{array}$ & Green liquor storage tanks & $\begin{array}{l}2 \text { each, } 17,500 \mathrm{gal}, 12 \mathrm{ft} \text { diam } \mathrm{x} \\
21 \mathrm{ft} \text { high, carbon steel, atmo- } \\
\text { spheric pressure }\end{array}$ & 3 \\
\hline$v-7$ & Spray nozzle test tank & $\begin{array}{l}3 \mathrm{ft} \text { diam } \times 10 \mathrm{ft} \text { high, } 15 \mathrm{lb} / \mathrm{in}^{2} \\
\text { (gage), } 250^{\circ} \mathrm{F} \text {, with view port, } \\
\text { carbon steel }\end{array}$ & 2 \\
\hline \multicolumn{4}{|c|}{ Pumps } \\
\hline$P-1$ & Black liquor recirculation pump & $\begin{array}{l}100 \mathrm{gal} / \mathrm{min}, 75-\mathrm{ft} \text { head, } 5 \mathrm{hp}, 480 \mathrm{~V} \text {, } \\
3 \mathrm{phase,} 1750 \mathrm{r} / \mathrm{min} \text {, double mechanical } \\
\text { seal, carbon steel }\end{array}$ & 2 \\
\hline $\mathrm{P}-2$ & Green liquor recirculation pump & $\begin{array}{l}100 \mathrm{gal} / \mathrm{min}, 170-\mathrm{ft} \text { head, } 10 \mathrm{hp}, 480 \mathrm{~V} \text {, } \\
3 \mathrm{phase,} 1750 \mathrm{r} / \mathrm{min} \text {, double mechanical } \\
\text { seal, carbon steel }\end{array}$ & 2 \\
\hline
\end{tabular}


Table 4. Equipment List

(Sheet 2 of 4 )

\begin{tabular}{|c|c|c|c|}
\hline & Item & Description & Source* \\
\hline$P-3$ & Black líquor return pump & $\begin{array}{l}20 \mathrm{ga} 1 / \mathrm{min}, 80-\mathrm{ft} \text { head, } 1-1 / 2 \mathrm{hp} \text {, } \\
480 \mathrm{~V}, 3 \text { phase, } 1750 \mathrm{r} / \mathrm{min} \text {, single } \\
\text { internal mechanical seal, carbon } \\
\text { steel }\end{array}$ & 2 \\
\hline$P-4$ & Black liquor feed pump & $\begin{array}{l}5 \mathrm{gal} / \mathrm{min}, 300-\mathrm{ft} \text { head, }\left(175 \mathrm{lb} / \mathrm{in}^{2}\right) \\
\sim 2 \text { hp, } 480 \mathrm{~V}, 3 \text { phase, } 304 \text { stain- } \\
\text { less steel rotary pump, variable } \\
\text { speed drive }\end{array}$ & 2 \\
\hline \multicolumn{4}{|c|}{ Compressors and Blowers } \\
\hline$c-1$ & Main feed air compressor & $\begin{array}{l}\text { Joy Twistair, } 470 \mathrm{cfm} \text { at } 100 \mathrm{1b} / \mathrm{in}^{2} \\
\text { (gage), 100-hp electric motor drive }\end{array}$ & 3 \\
\hline$c-2$ & Back-up feed air compressor & $\begin{array}{l}\text { Ingersol-Rand vane type } \\
\text { compressor, } 365 \mathrm{cfm} \text { at } 100 \mathrm{~b} / \mathrm{in}^{2} \\
\text { (gage), diesel engine drive }\end{array}$ & 3 \\
\hline$c-3$ & Combustor air fan & $\begin{array}{l}\text { North American Turbo Blower, 2332- } \\
21-2-30,2000 \text { cfm at } 2 \text { lb/in? } \\
\text { (gage), } 30 \mathrm{hp}\end{array}$ & 3 \\
\hline \multicolumn{4}{|c|}{ Filters and Strainers } \\
\hline$S-1$ & Baghouse & $\begin{array}{l}\text { Mikropul Model } 495-8-20 \text {, stainless } \\
\text { with } 3-\text { in. insulation, Nomex } 14-0 \mathrm{z} \\
\text { felt bags, } 500 \mathrm{ft}^{2}\end{array}$ & 4 \\
\hline$S-2$ & Black liquor loop strainer & $\begin{array}{l}\text { Rotary strainer, Dorr 0liver, } 4 \text { in., } \\
9 \mathrm{r} / \mathrm{min}, 1 / 4 \mathrm{hp}\end{array}$ & 2 \\
\hline$S-3$ & Black liquor feed strainer & $\begin{array}{l}\text { Rotary strainer, Dorr 0liver, } 2 \text { in., } \\
16 \mathrm{r} / \mathrm{min}, 1 / 8 \mathrm{hp}\end{array}$ & 2 \\
\hline$S-4$ & Black liquor unloading strainer & $\begin{array}{l}\text { In-line } 3 \text {-in. pipe strainer, Y-type, } \\
\text { coarse screen }\end{array}$ & 2 \\
\hline$S-5$ & Green liquor product strainer & In-line 1 -in. pipe strainer, Y-type & 2 \\
\hline$S-6$ & Quench tank strainer & $\begin{array}{l}\text { Circulation pump inlet strainer, } \\
\text { coarse screen, in tank }\end{array}$ & 2 \\
\hline
\end{tabular}

$A I-D O E-13566$ 
Table 4. Equipment List

(Sheet 3 of 4 )

\begin{tabular}{|c|c|c|c|}
\hline \multicolumn{2}{|r|}{ Item } & Description & \multirow{2}{*}{$\frac{\text { Source* }}{2}$} \\
\hline$S-7$ & Black liquor tank vent filter & $\begin{array}{l}\text { Activated carbon, replaceable } \\
\text { canister type, for odor removal, } \\
\text { j-in. pipe connections, l-atm } \\
\text { pressure }\end{array}$ & \\
\hline$S-8$ & Nozzle test tank vent filter & $\begin{array}{l}\text { Activated carbon, replaceable } \\
\text { canister type, for odor removal, } \\
\text { 1-in. pipe connections, l-atm } \\
\text { pressure }\end{array}$ & 2 \\
\hline $\begin{array}{l}S-9 \\
A \& B\end{array}$ & $\begin{array}{l}\text { Green liquor storage tank vent } \\
\text { filters }\end{array}$ & $\begin{array}{l}\text { Activated carbon, replaceable } \\
\text { canister type, for odor remova1, } \\
1 \text {-in. pipe connections, 1-atm } \\
\text { pressure }\end{array}$ & 2 \\
\hline \multicolumn{4}{|c|}{ Heaters, Coolers, and Burners } \\
\hline$H-1$ & Product gas combustor & $\begin{array}{l}\text { Custom J. T. Thorpe, Inc., rated } \\
600-\text { scfm product gas at } 150 \mathrm{Btu} / \mathrm{scf} \text {, } \\
\text { in let temperature } 1600 \text { to } 2000^{\circ} \mathrm{F}\end{array}$ & 3 \\
\hline$H-2$ & Feed air heater & $\begin{array}{l}180 \mathrm{~kW}, 480 \mathrm{~V}, 3 \text { phase, in-line with } \\
\text { SCR controller, } 1101 \mathrm{~b} / \text { in } 2 \text { (gage), } \\
\text { at } 1100^{\circ} \mathrm{F} \text { and } 151 \mathrm{~b} / \text { in2 (gage) at } \\
1500^{\circ} \mathrm{F} \text {. ASME Section VIII, Hynes } \\
\text { Electric Heating Company }\end{array}$ & 3 \\
\hline$H-3$ & Melt discharge line burner & $\begin{array}{l}75,000 \mathrm{Btu} / \mathrm{h}, \text { natural gas, } 2000 \% \\
\text { excess air to } 30 \% \text { excess fuel, } \\
85-7 \mathrm{~b} / \text { in2 (gage) operating } \\
\text { pressure }\end{array}$ & 2 \\
\hline$H-4$ & Black liquor tank heater & $\begin{array}{l}40 \mathrm{~kW}, 480 \mathrm{~V}, 3 \text { phase, in tank } \\
\text { heater with SCR controller, } 0 \text { to } \\
250^{\circ} \mathrm{F}, 8-\text { in. } A 300 \text { flanges }\end{array}$ & 2 \\
\hline$H-5$ & $\begin{array}{l}\text { Black liquor recirculation } \\
\text { heater }\end{array}$ & $\begin{array}{l}40 \mathrm{~kW}, 480 \mathrm{~V}, 3 \text { phase, in-line } \\
\text { heater with SCR controller, } 0 \text { to } \\
250^{\circ} \mathrm{F}, 2-i n . A 300 \text { flanges }\end{array}$ & 2 \\
\hline$H-6$ & Green liquor heater & $\begin{array}{l}15 \mathrm{~kW}, 480 \mathrm{~V}, 3 \text { phase, in-line } \\
\text { heater with SCR controller, } 0 \text { to } \\
250^{\circ} \mathrm{F}, 2 \text { in., A300 flanges }\end{array}$ & 2 \\
\hline$H-7$ & Vessel preheat burner A & $\begin{array}{l}75,000 \text { Btu/h, natural gas, } 2000 \% \\
\text { excess air to } 30 \% \text { excess fue } 1 \text {, } \\
85-1 b / i n 2 \text { (gage) operating } \\
\text { pressure }\end{array}$ & 2 \\
\hline
\end{tabular}


Table 4. Equipment List

(Sheet 4 of 4 )

\begin{tabular}{|c|c|c|c|}
\hline & Item & Description & Source* \\
\hline$H-8$ & Green liquor cooler & $\begin{array}{l}\text { Shell and tube, stainless tubes, } \\
150,000-B \text { tu/h cooling, } 70^{\circ} \text { cooling } \\
\text { water }\end{array}$ & 2 \\
\hline$H-9$ & Product gas cooler & $\begin{array}{l}6-15 \mathrm{ft} \text { lengths of } 8 \text { in. Schedule } 20 \\
\text { carbon steel pipe, spaced } 2 \mathrm{ft} \text { apart } \\
\text { in vertical parallel arrangement, } \\
\text { with interconnecting fittings, } \\
\text { bypass, and drains }\end{array}$ & 1 \\
\hline $\mathrm{H}-10$ & Vessel preheat burner $B$ & $\begin{array}{l}\sim 1 \mathrm{million} \text { Btu/h oil burner, } \\
\text { maximum capacity } 72 \mathrm{~kg} / \mathrm{h}(159 \mathrm{lb} / \mathrm{h}) \text {, } \\
20,000 \mathrm{Btu} / 1 \mathrm{~b} \text { oil, normal operation } \\
18 \mathrm{~kg} / \mathrm{h}(40 \mathrm{lb} / \mathrm{h}) \text { oil and } 800 \mathrm{lb} / \mathrm{h} \\
\text { air }(50 \% \text { excess })\end{array}$ & 2 \\
\hline \multicolumn{4}{|c|}{ Agitators } \\
\hline$A-1$ & Black liquor tank agitator & $\begin{array}{l}6 \mathrm{hp}, 300 \mathrm{r} / \mathrm{min} \text {, dual-propeller } \\
\text { stainless with double mechanical } \\
\text { seal, } 8 \text { in. Al50 flange top entry, } \\
50-1 \mathrm{~b} / \mathrm{in}^{2} \text { (gage) operation }\end{array}$ & 2 \\
\hline$A-2$ & Quench tank agitator & $\begin{array}{l}6 \text { hp, } 300 \mathrm{r} / \mathrm{min} \text {, dual-propeller } \\
\text { stainless with double mechanical } \\
\text { seal, } 8 \text { in. Al50 flange top entry, } \\
150 \text {-ib/in }{ }^{2} \text { (gage) operation }\end{array}$ & 2 \\
\hline \multicolumn{4}{|c|}{ Special Hardware } \\
\hline $2-1$ & Soot blower & $\begin{array}{l}\text { Nitrogen or steam feed, pneumatic } \\
\text { drive reciprocating hollow shaft, } \\
\text { i in. OD, 9-ft thrust, local } \\
\text { control }\end{array}$ & 4 \\
\hline
\end{tabular}

*Equipment Sources:

1 - Special equipment, to be designed and fabricated

2 - Standard equipment, to be purchased

3 - Existing Rockwel1 equipment, relocation, refurbishing, and installation may be required

4 - Existing DOE equipment, requires DOE approval for use. Relocation, refurbishing, and installation required.

$1603 \mathrm{D} / \mathrm{rmr}$ 
are the two feed air compressors, the feed air heater, and the product gas combustor, including its air fan. Two existing large tanks have also been earmarked for use in the pilot plant as storage tanks for green liquor. These tanks will require relocation. The list does not cover instrumentation and control items, which represent an important part of the existing facility to be used in the pilot plant. These are described in a later section.

4. Existing DOE equipment. At least two items of equipment currently installed on an inactive DOE-owned facility adjacent to the pilat plant site can be effectively used in the black liquor gasification pilot plant. These are the baghouse and soot blower. The latter was designed specially for operation in a pressurized gasifier product gas outlet line.

\section{Gasifier}

The gasifier represents the key element of the process, and considerable effort has gone into its design. As presently configured, the gasifier is a carbon steel pressure vessel, $5 \mathrm{ft} 7-1 / 2 \mathrm{in}$. OD and about $24 \mathrm{ft} 6$ in. total height. The walls are lined with a 2-in. layer of bubble alumina insulating refractory, an 8-in. layer of dense alumina refractory, and, in the bottom portion, an 8-in. layer of fusion cast alumina blocks. The selected refractories are manufactured by the Carborundum Company and are designated Alfrax B1, Alfrax 66, and Monofrax $M$, respectively. The gasifier is shown in Figure 5 (engineering drawing $M 005-68964-M 3$ ) and the refractory configuration within the vessel is shown in Figure 6 (engineering drawing M005-68964-M4).

The basic configuration and functions of the gasifier are unchanged from the previous design, which has been described in detail in prior reports. 5,7 The major changes which have been made are represented in the following: .

1. The design pressure has been increased to $150 \mathrm{lb} / \mathrm{in}^{2}$ (gage) (for operation at $90 \mathrm{lb} / \mathrm{in}^{2}$ (absolute) instead of $45 \mathrm{lb} / \mathrm{in}^{2}$ (absolute)).

2. The lower melt discharge nozzle has been deleted. 

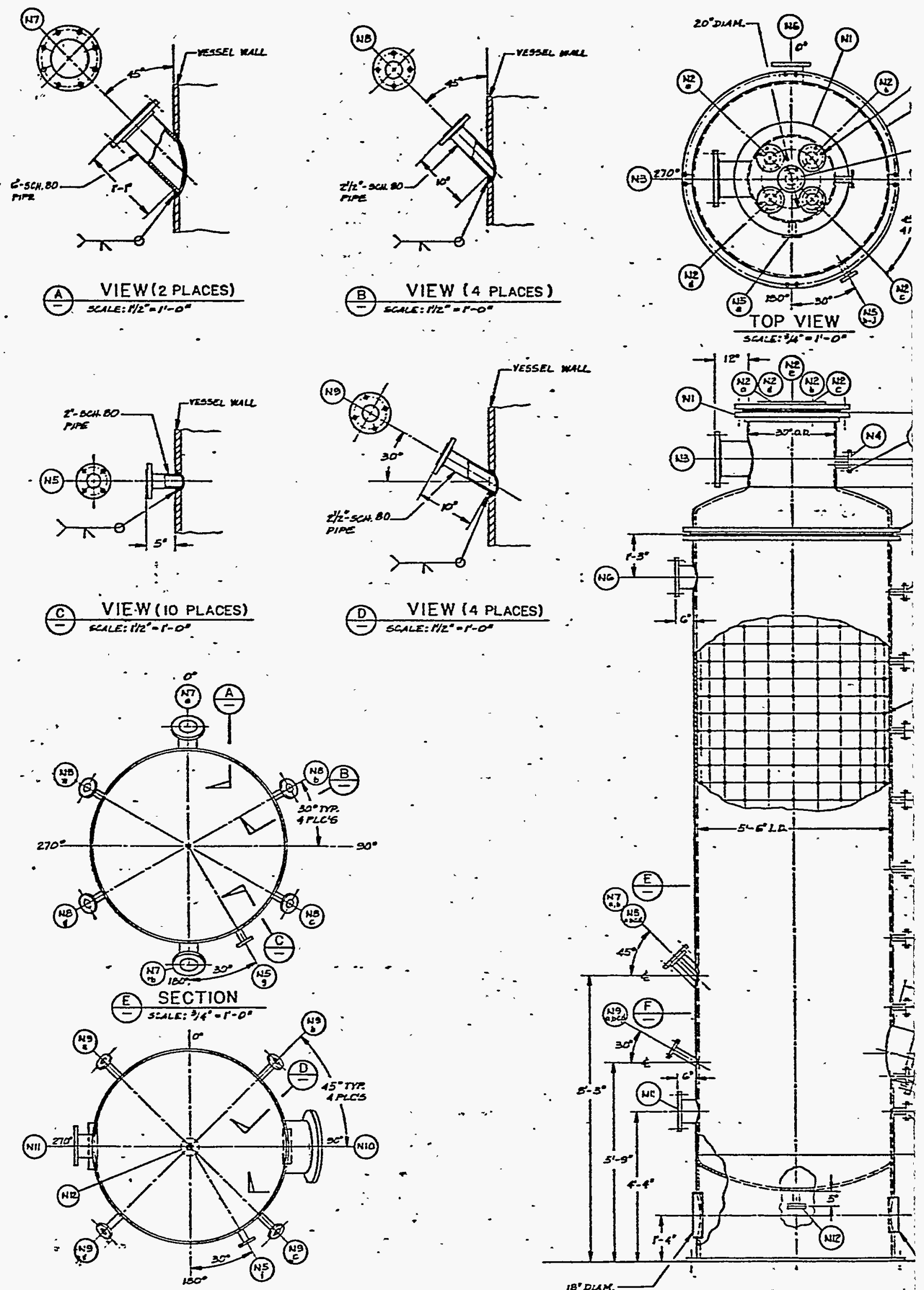

$=$ F SECTION

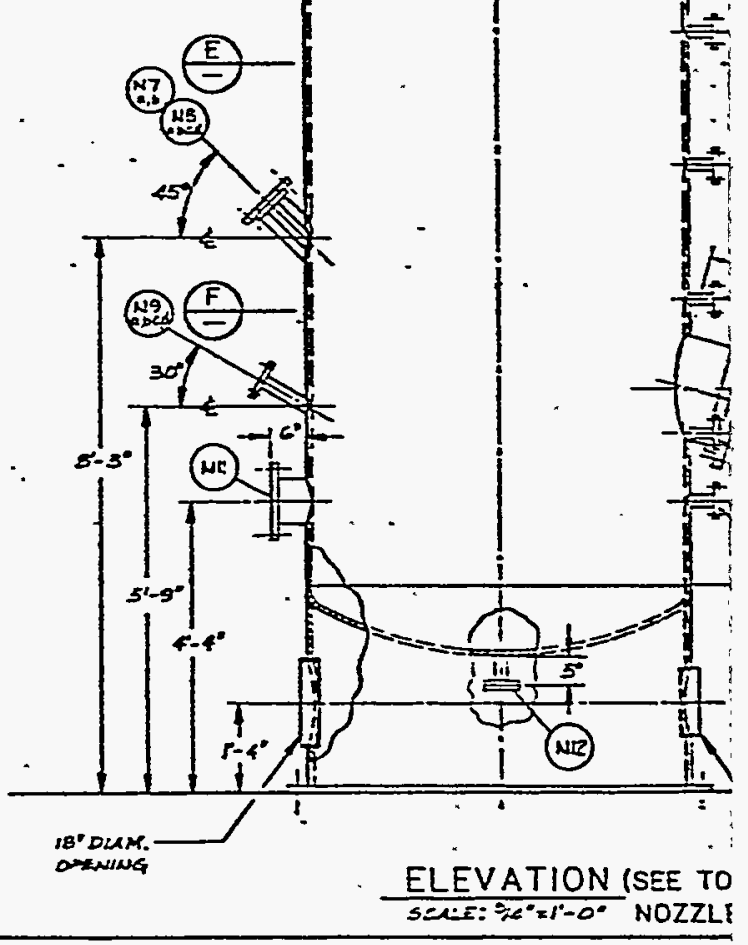




\begin{tabular}{|c|c|c|c|c|c|c|}
\hline \multicolumn{7}{|c|}{ NCZZLE SXXEDULE } \\
\hline 2 & DESERIPTION & seg & TrPe & entue & $\rho m$ & esuecess \\
\hline 1 & WENO DTELETON & so? & SEPP-ON & 250 & 1 & \\
\hline 2 & HEAD FORT & 4 & acinto & 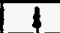 & 5 & $a, b, c, d, e$ \\
\hline 3 & Peosure ans & 12 & serpas & & 1 & \\
\hline 4 & coor BLOVER LEOLLE & 2 & 1 & & 1 & \\
\hline 5 & TC/UTIECOR CASHFIER & 20 & & & 10 & $a, b, c, d, f, g, b, \bar{i}, J]$ \\
\hline 6 & PESSURE RLIEF & $10^{\circ}$ & & & 1 & \\
\hline 7 & 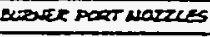 & 5 & & & 2 & asb \\
\hline 8 & Wher wi MEZLES & $2 / 2$ & & & 4 & $a, b, c, d$ \\
\hline 9 & LOWER AIR NEZZLES & एक & & & 4 & a.b.c.d \\
\hline 10 & 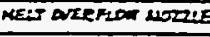 & 18 & & & 1 & \\
\hline$t$ & DeNA NCEZLE & $\theta^{n}$ & & & 1 & \\
\hline 12 & MD:STWRE VENT & 2 & serpas & 1 & 1 & \\
\hline $1 B$ & NENO RAWE & 65 & & 150 & & \\
\hline
\end{tabular}

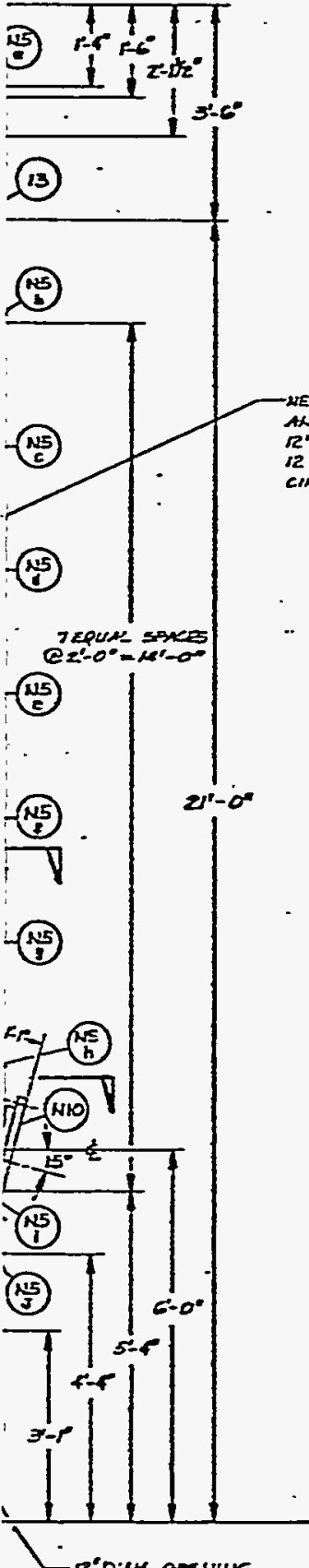

MELSON EEFRACTOE ANGHDe, STHE VXCO R'O.C. VERTRAL UD CIRCUMFERENCE.

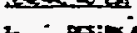

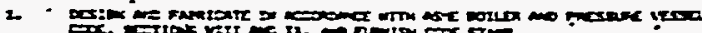

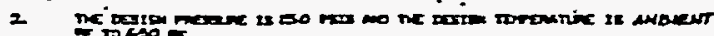

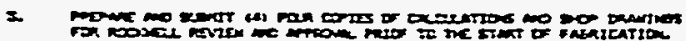

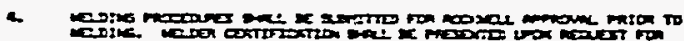

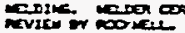

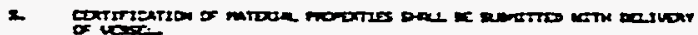

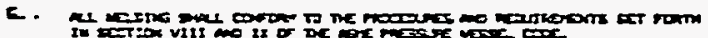

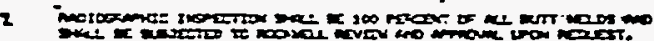

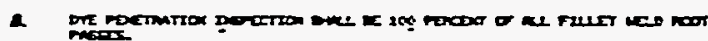

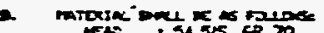

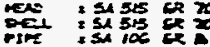

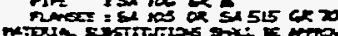

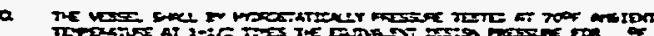

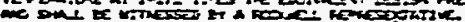

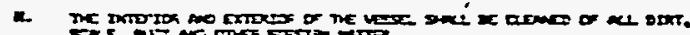

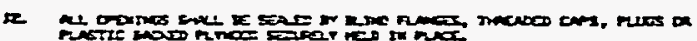

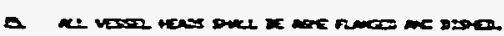

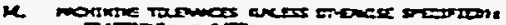

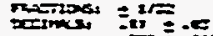

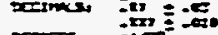

$\operatorname{sen} 20$

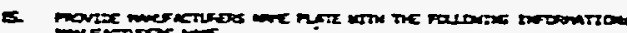
mexpesias

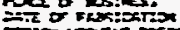

sid weric meser-

ex mines

on mise

E. LO

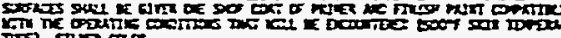

AI-DOE-13566 29

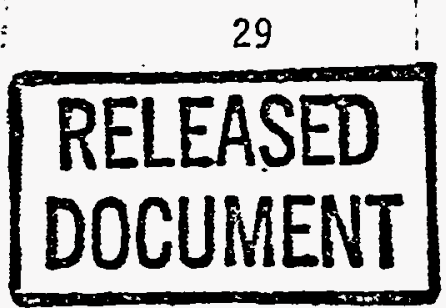

$\frac{\text { CONCEPTUAL DRDWNK }}{\text { MET FOR CONSTENTION }}$

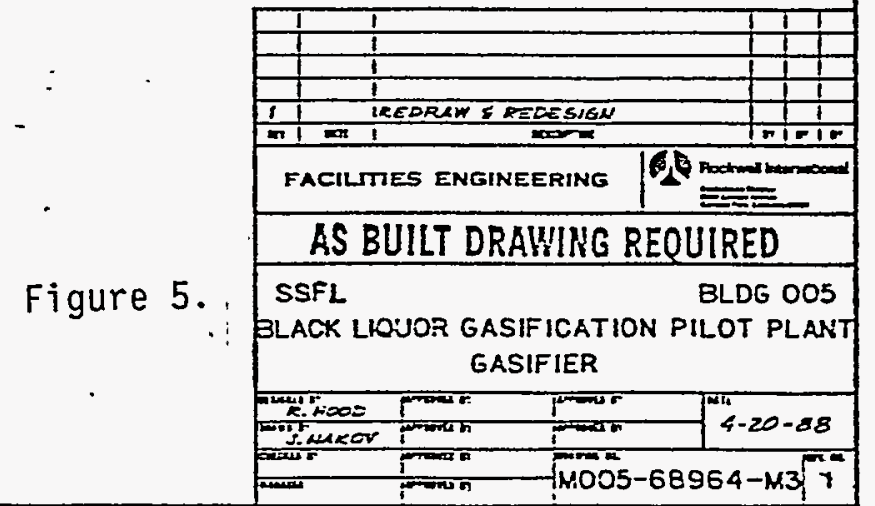

FILE NO 503-CO5-M74 


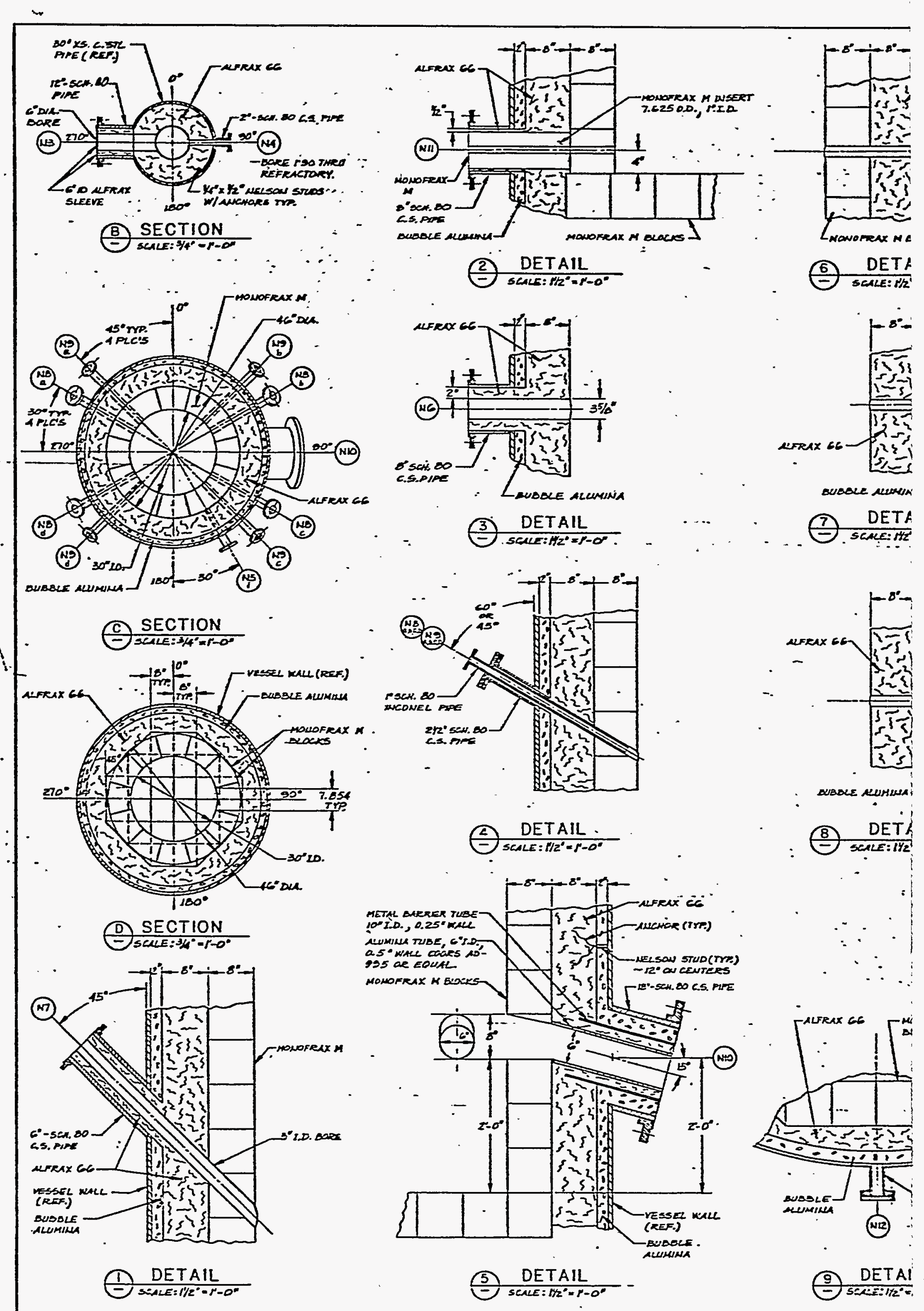


3. The air feed nozzles have been relocated and increased in size.

4. New nozzles have been added for preheat burners (2), drain, and pressure relief.

5. Thermowell nozzles have been relocated.

6. Refractory installation details have been developed for all nozzles.

In the previous design, two melt discharge nozzles were provided at different heights so that the effect of melt depth could be evaluated. However, use of the second discharge nozzle would require rotating the gasifier vessel $180 \mathrm{deg}$ and raising (or lowering) the quench tank subsystem to match the new nozzle height. In the present design, the effect of melt. depth can be evaluated by installing a layer of filler blocks on the gasifier floor, a much simpler and more economical procedure. Hot product gas leaves the top of the gasifier at temperatures up to $921^{\circ} \mathrm{F}$ during normal operation at reference design conditions; however, it is estimated that gas temperatures as high as $1200^{\circ} \mathrm{F}$ may occur during off-normal operating periods. The gasifier outlet line is, therefore, refractory lined. The product gas flows into the separator shown in Figure 7 (drawing M005-6894-M5). Because of the high operating temperature, the material specified for this unit is Type 304 or 316 stainless steel. Design conditions for the separator are $1200^{\circ} \mathrm{F}$ and $150 \mathrm{lb} / \mathrm{in}^{2}$ (gage). A 6-in. diameter stainless steel liner is provided in the 8-in. gas entry nozzle of the separator to minimize thermal effects at this point. It is anticipated that the product gas will cool approximately $100^{\circ} \mathrm{F}$ while proceeding through the uninsulated separator vessel.

\section{Quench System}

The melt discharge line and quench tank designs have been modified to accommodate the higher operating pressure and the new fixed elevation of the melt discharge nozzle. They are shown in Figures 8 and 9 (drawings M005-68984M6 and 174000003, respectively).

AI-DOE- 13566 
One significant change in the quench tank design is the addition of a stainless steel liner in the vertical extension of the main tank. The liner is spaced about 2 in. from the vessel wall and is designed to protect the portion of the wall that is directly in line with the melt discharge port from direct contact with high temperature melt and gases. Both the liner and unlined portions of the vertical extension will be continuously washed with recycled green liquor during operation to provide additional protection.

Gas entering the quench tank will be cooled and washed by contact with the wall wash liquid and the shatter jet spray. The gas will flow from the quench tank to a small separator, which will remove entrained droplets of green liquor or other particles. The design of this separator is shown in Figure 10 (drawing M005-68964-M8).

It is planned that pressure vessels in the pilot plant will be designed and fabricated in accordance with the ASME Code, Section VIII, which requires that corrosion be considered in the design. The quench tank and vent gas separator will be contacted by green liquor, water, and gas during plant operation. Hot green liquor is believed to represent the most corrosive medium that will be encountered. The current maximum design operating temperature is $220^{\circ} \mathrm{F}$. The green liquor temperature can be prevented from exceeding $220^{\circ} \mathrm{F}$ (or reduced to a lower value if desired) by operation of the green liquor cooler, injection of more makeup water, or reducing the flow of melt and/or hot gas from the gasifier. The vent gas separator is expected to operate at the same temperature as the quench tank; however, it can be cooled independently by the injection of makeup water through a nozzle provided at the gas inlet port.

The corrosion of metals in green liquor was evaluated by Rocketdyne in a previous program. ${ }^{8}$ Although the green liquor used was not identical to that which will be encountered in the black liquor pilot plant program, it was quite similar. The reported test results for carbon steel and Type 304 stainless steel are given in Table 5. To estimate the corrosion rate at $220^{\circ} \mathrm{F}$, the carbon steel data are plotted on semilog paper in Figure 11 . 

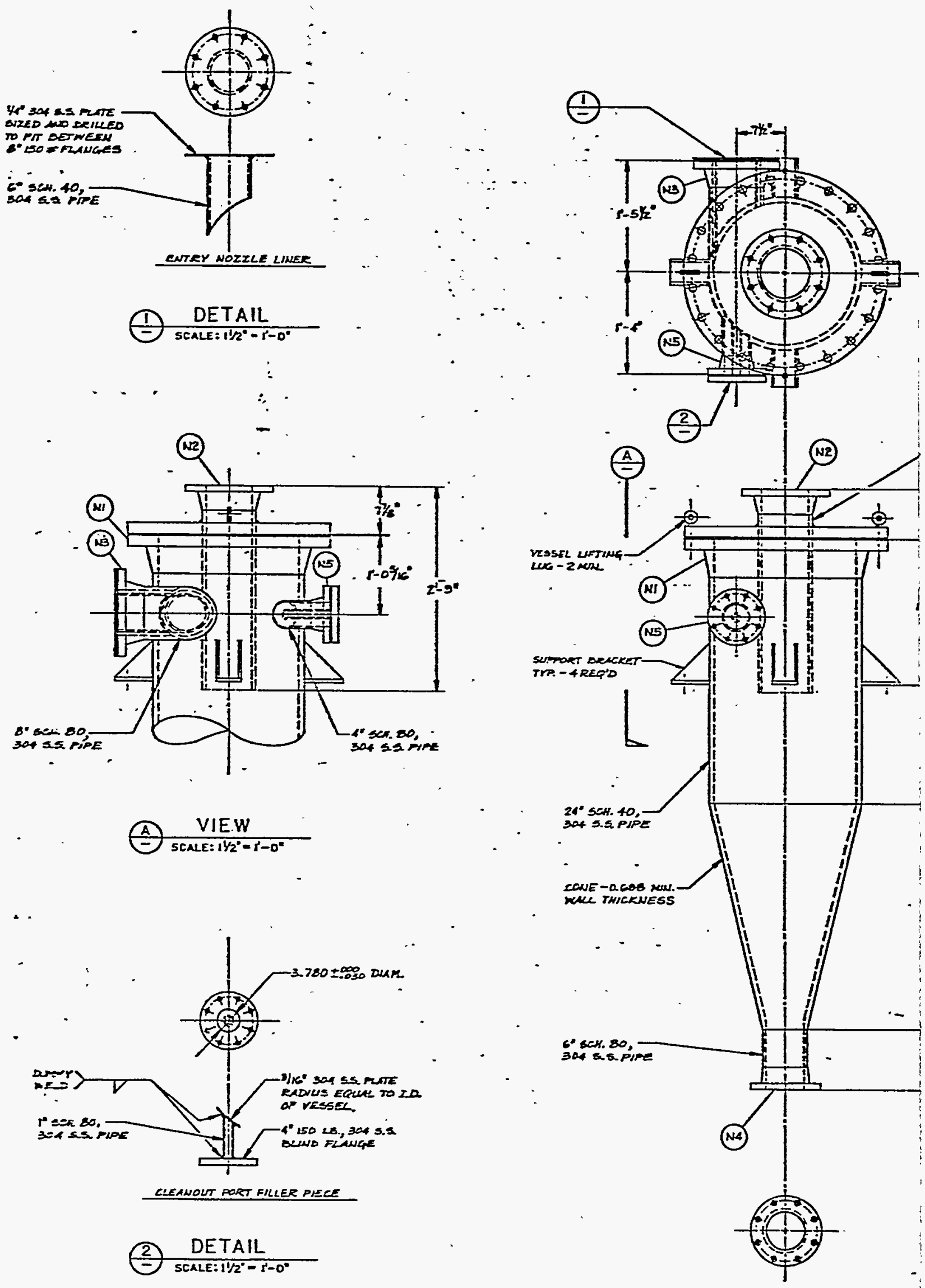

SCALE: $t^{2} 2^{\circ}=t^{2}-0^{\circ}$ 


\begin{tabular}{|c|c|c|c|c|c|c|}
\hline \multicolumn{7}{|c|}{ NOZZLE SCHEDULE } \\
\hline (a) & DESCRIPTION & 50 & TYPE & EATL4 & का & ENuRES \\
\hline $\boldsymbol{I}$ & NEAD RUAKE & $3+1$ & KNe. & 250 & 11 & \\
\hline 2 & SAS QUTLET & 8 & & & II & \\
\hline $\mathbf{3}$ & ENTER NOZZLE & 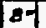 & & & 1 & \\
\hline 4 & SXIDSS DISCHARGE & 67 & & & (1) & \\
\hline 5 & CENWDUT PART & 14 & & t & 1 & \\
\hline
\end{tabular}

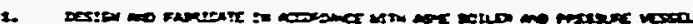

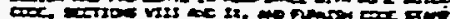

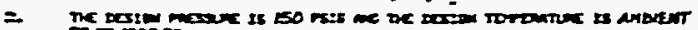

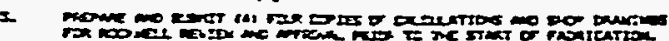

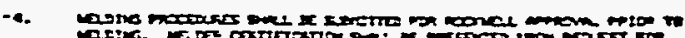

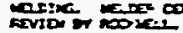

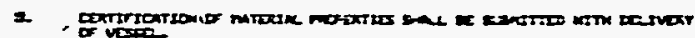

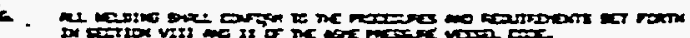

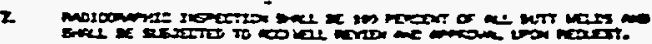

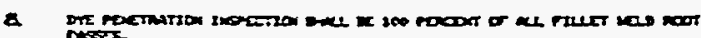

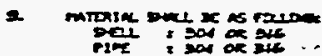

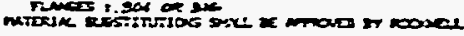

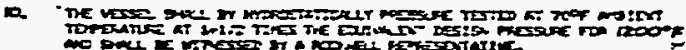

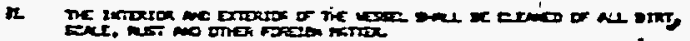

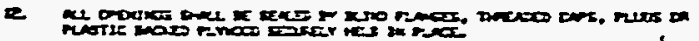

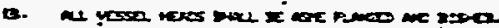

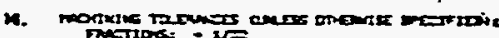

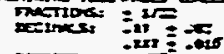

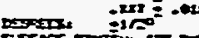

s.

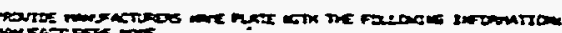
mertines

Date of FATERTizon

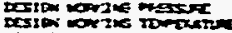

Die nesces

cien niv:

/2

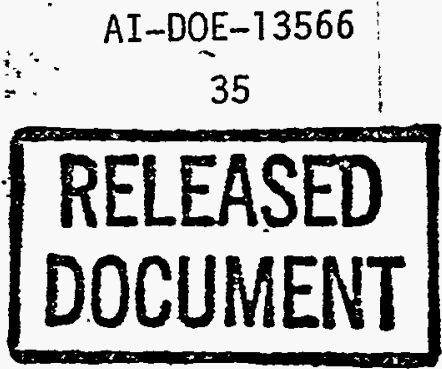

CONEEPTUA: DRMNIN

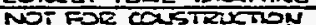

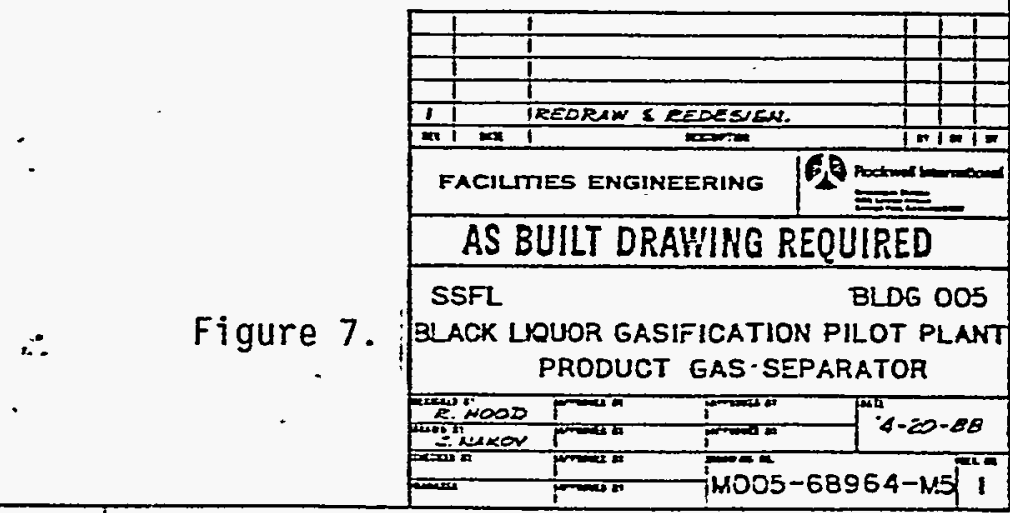




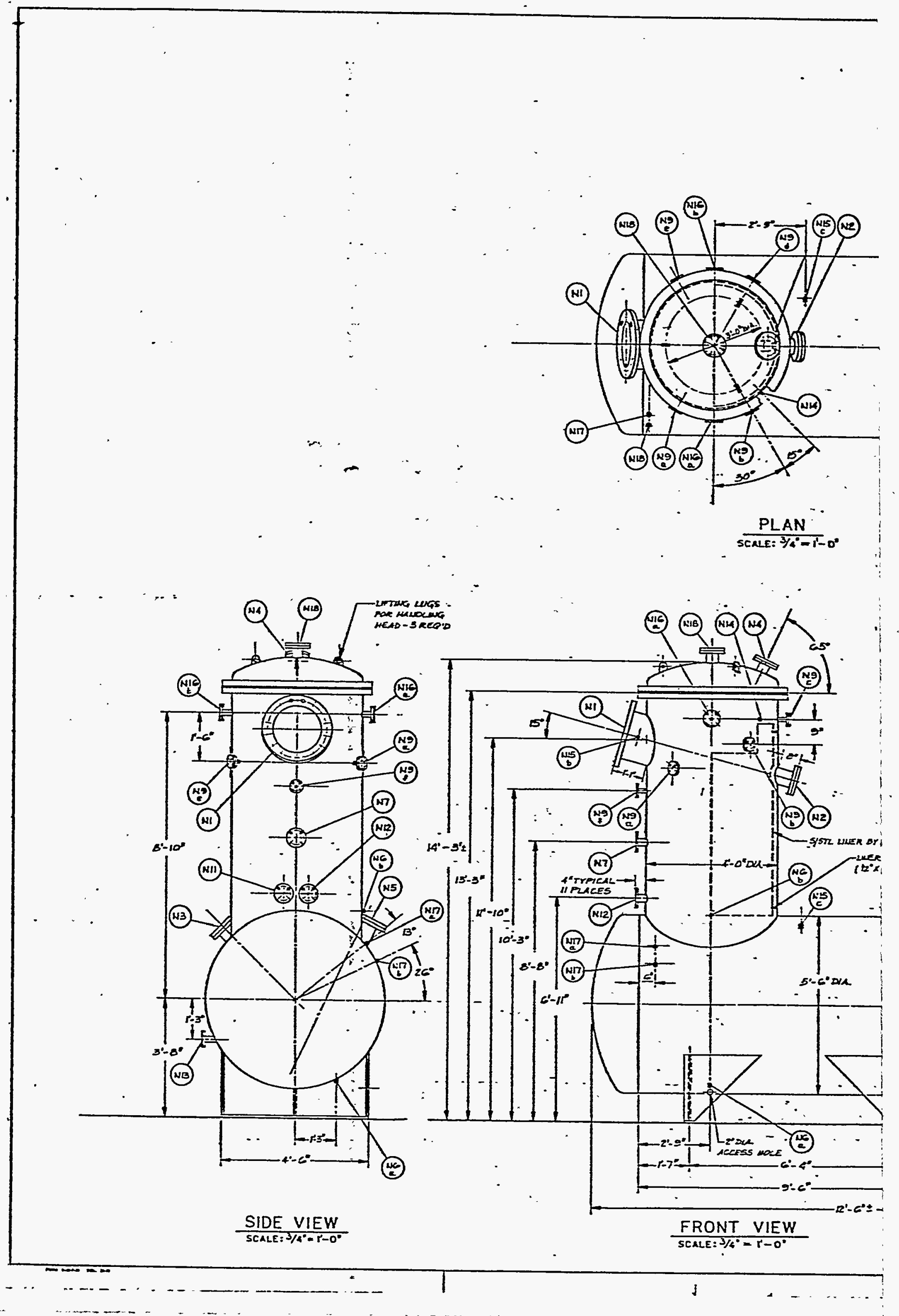



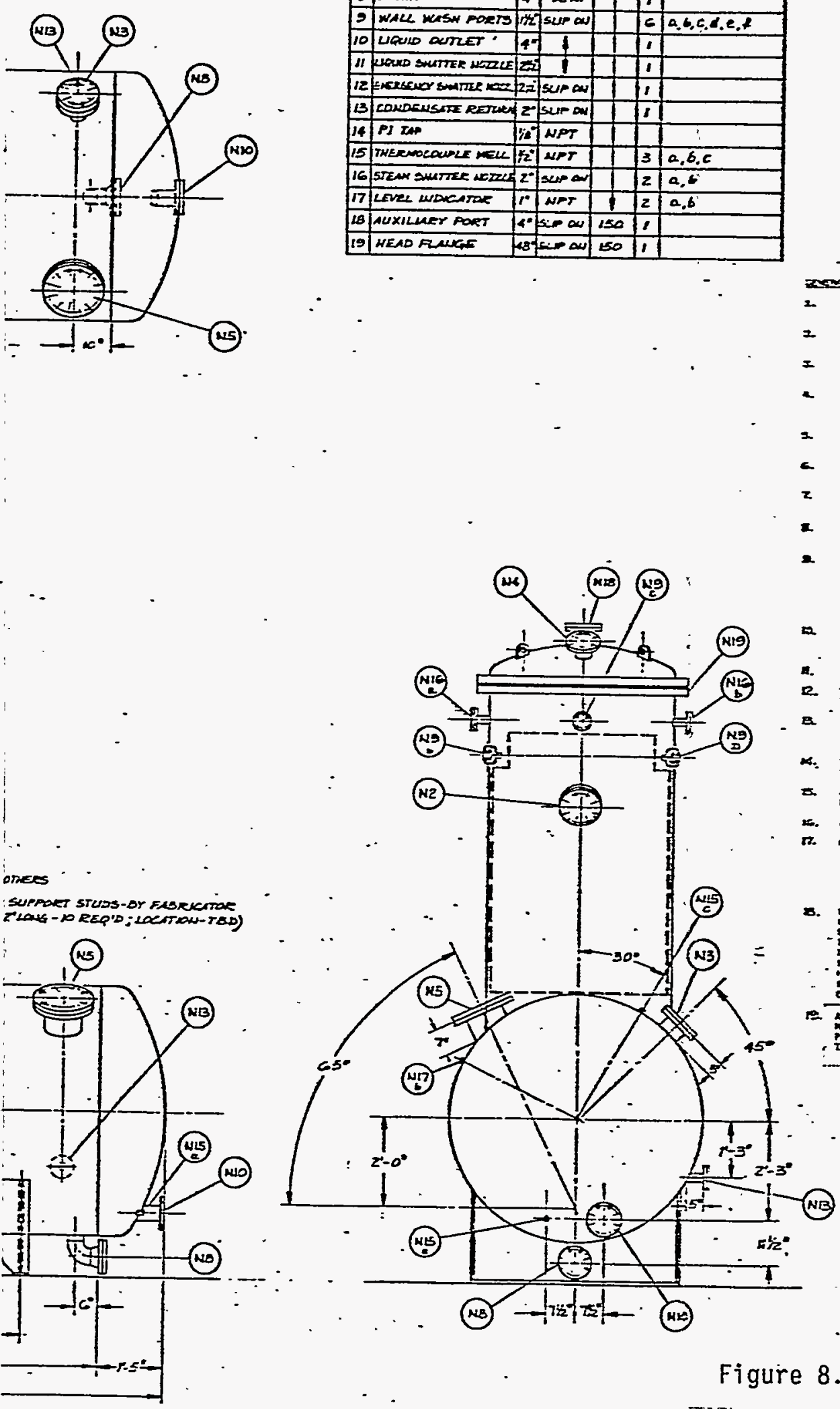

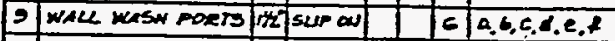

NOZZLE SCHEDOLE

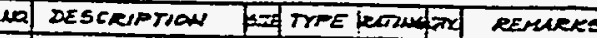

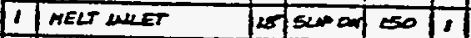

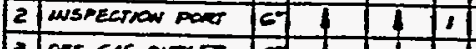

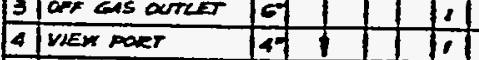

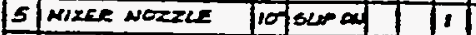

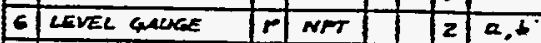

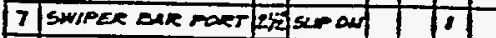

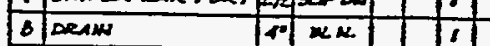

\begin{tabular}{|l|l|l|}
\hline 10 & LQWD aUTEr' & 47
\end{tabular}

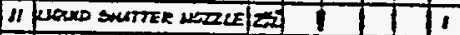

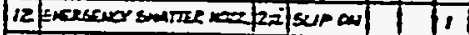

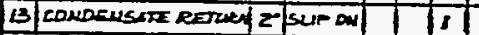

14 PI zie

Vie' $\mathrm{NPT}$

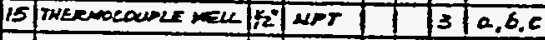

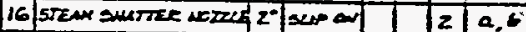

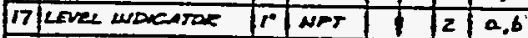

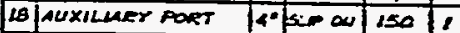

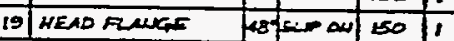

Figure 8.

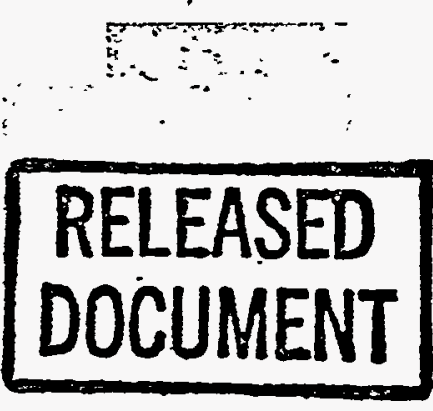

$\operatorname{xan}$

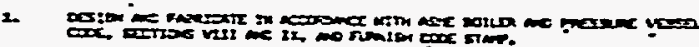

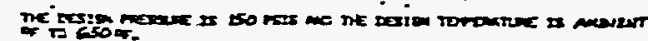

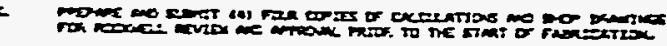

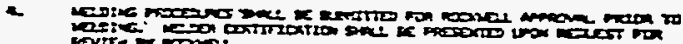

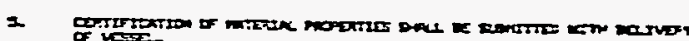

- 21

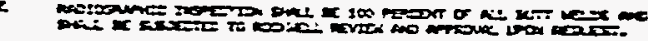

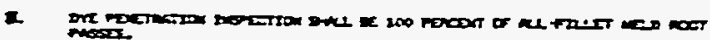

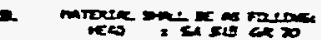

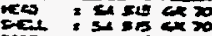

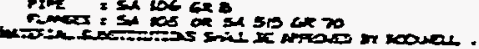

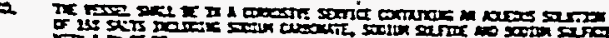
and to

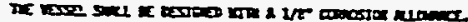

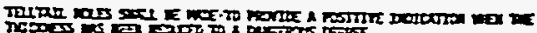

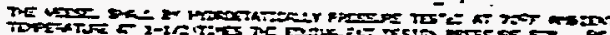

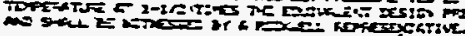

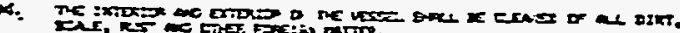

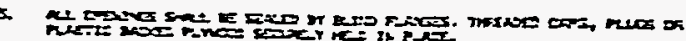

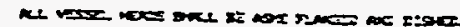

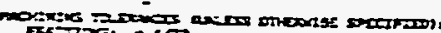

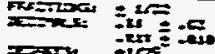
sifin macion

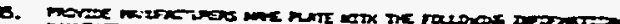

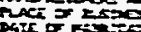

$\cos 20$

-

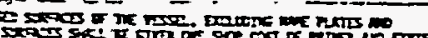

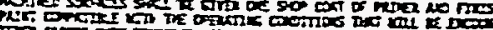

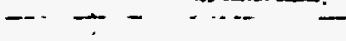
$\frac{\text { CONCEPTUAL DEAWING }}{\text { NOT DOE CONSTRUCTION }}$

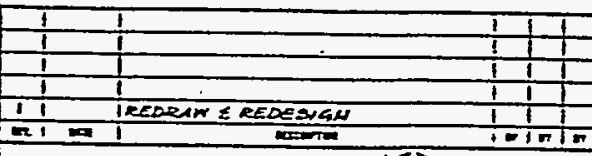

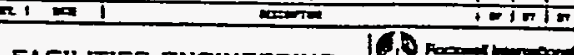

FACILTIES ENGINEERING AS BUILT DRAWING REOUIRED

SSFL B BDDJ 005 BLACK LIQUOR GASIFICATION PILOT PLANT QUENCH TANK

\begin{tabular}{|c|c|c|c|}
\hline$\sum_{n=000}$ & $!=00$ & Prenta & \\
\hline & 1 & pen & \\
\hline & $\pi$ & $m$ & \\
\hline
\end{tabular}




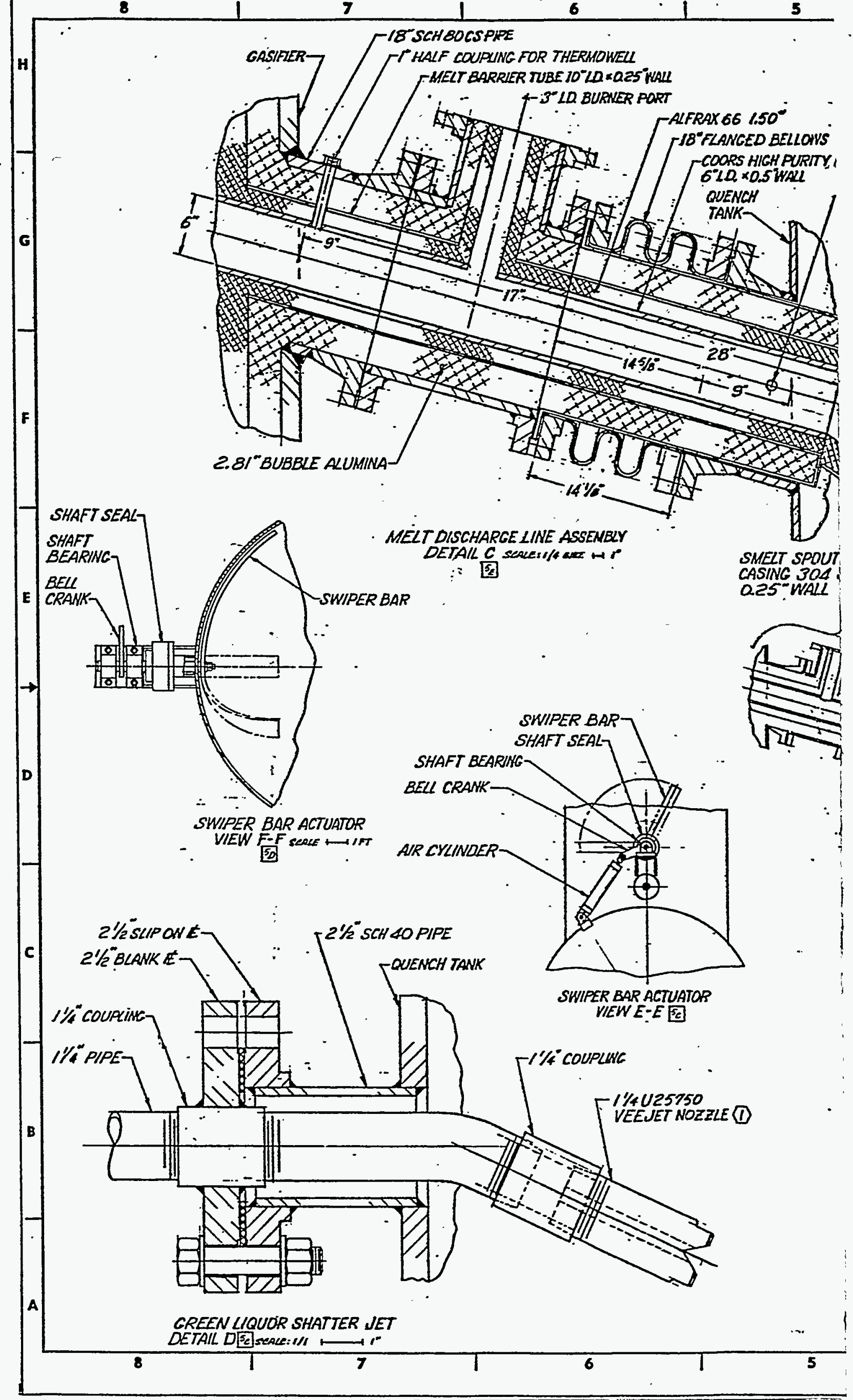




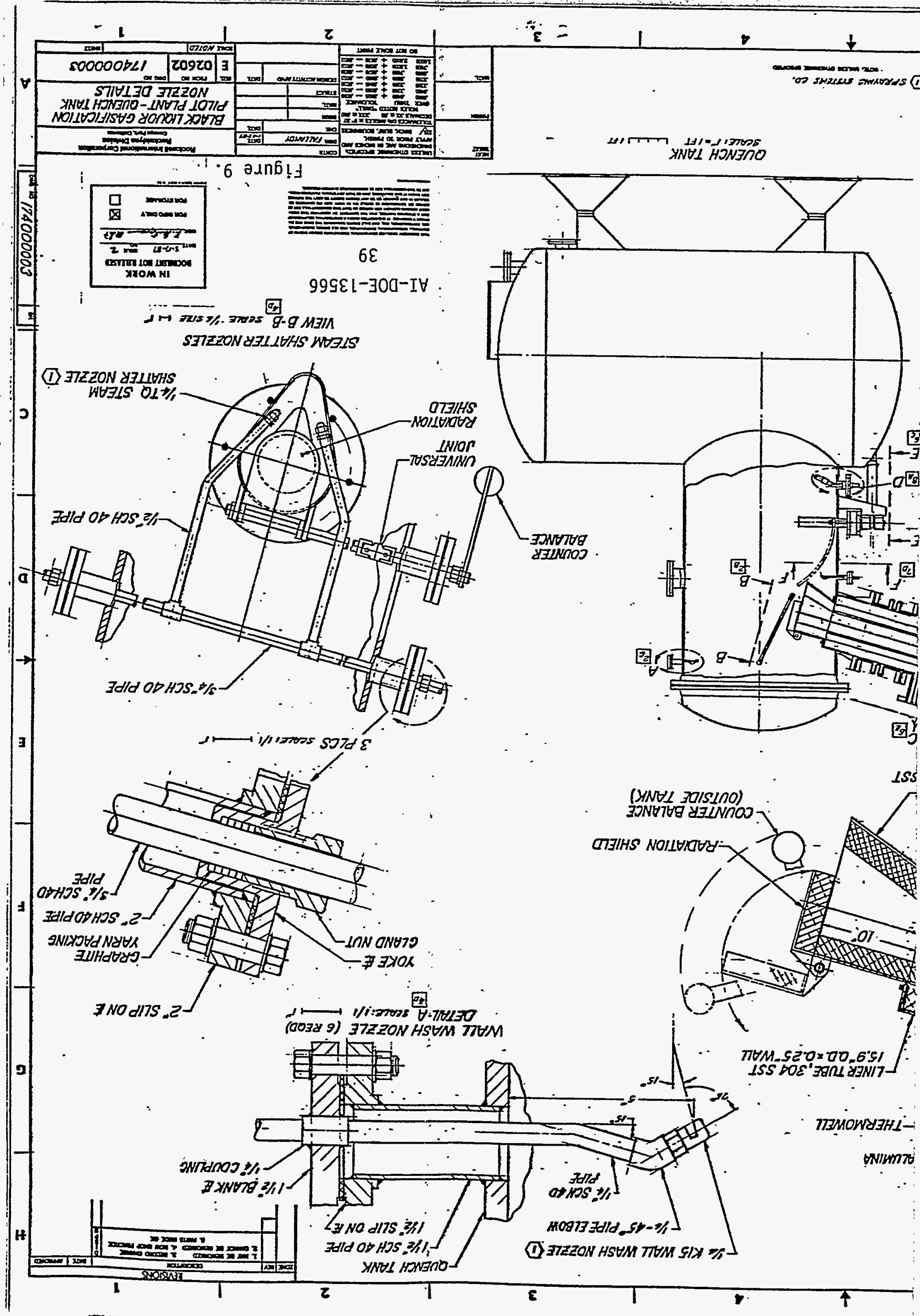




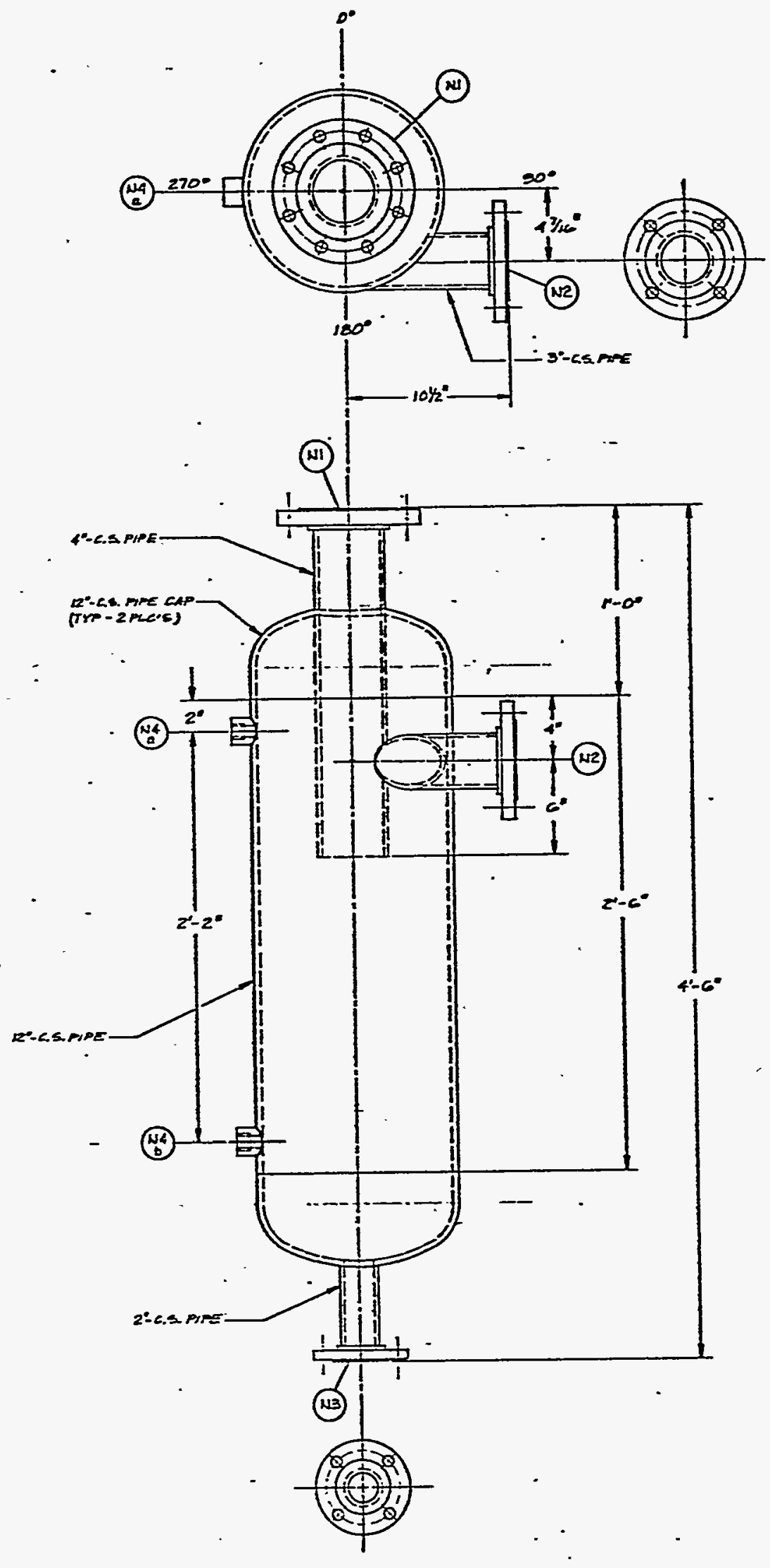

SCALE: $3^{\circ}-1^{\prime}-0^{\circ}$ 


\begin{tabular}{|c|c|c|c|c|c|c|}
\hline \multicolumn{7}{|c|}{ NOZZLE SCHEDULE } \\
\hline un & DESCEIPTION & Size & TMPE & errise & $\Rightarrow$ & AEMARES \\
\hline 1 & CAS QNTLE & $14^{\circ}$ & $\sin 0 x$ & 250 & 1 & \\
\hline 2 & INLET & $3^{\circ}$ & 5en-an & 50 & 1 & \\
\hline 3 & DRAIN & $2{ }^{*}$ & sip-a & 150 & 1 & $\omega$ \\
\hline 4 & LEYEL ADICATOR & $r^{\prime \prime}$ & NPT & 5000 & 2 & $a, b(c 0 u r(2) a)$ \\
\hline
\end{tabular}

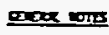

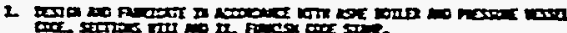

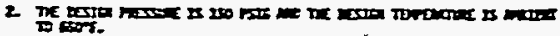

2 .

4. in toon

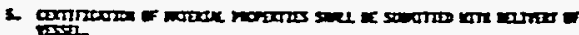

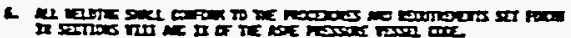

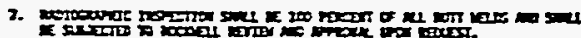

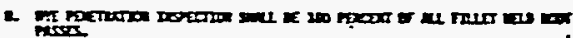

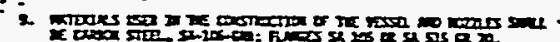

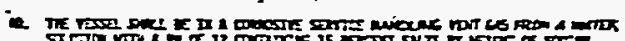

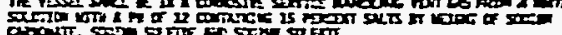
ascons

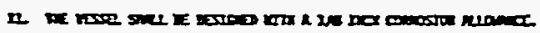

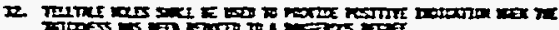

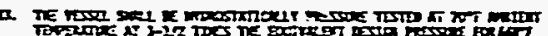

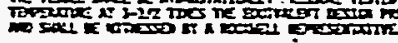

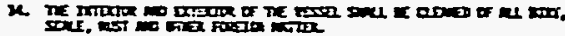

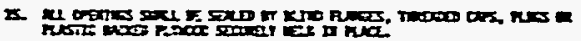

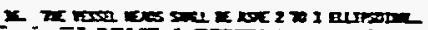

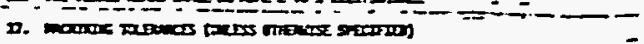
Pxicis:

mexis

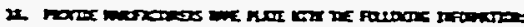

porcasis

xactions

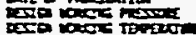

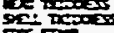

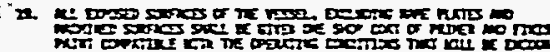

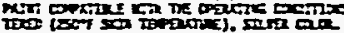

- - .

AI-DOE-13566

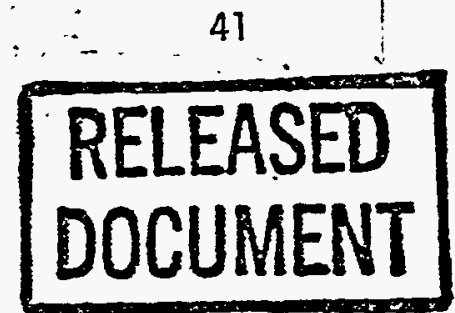

CONCEPTIAL DRAWING

NOT FOE COLSTRECTLN

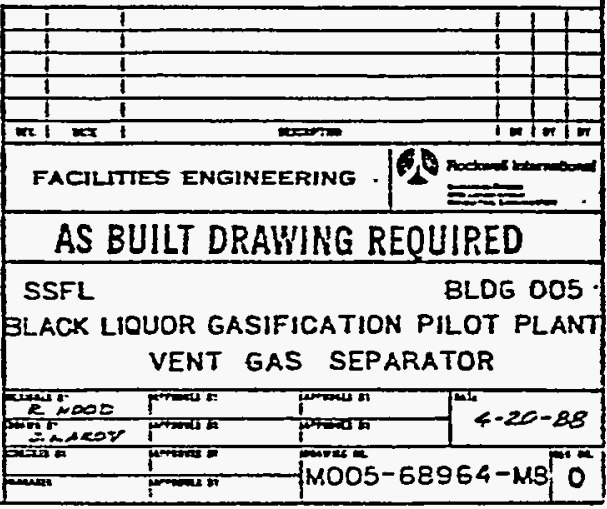

Figure 10 . BLACX LIOUOR GASIFICATION PILOT PLANT 
Table 5. Corrosion of Metals in Green Liquor*

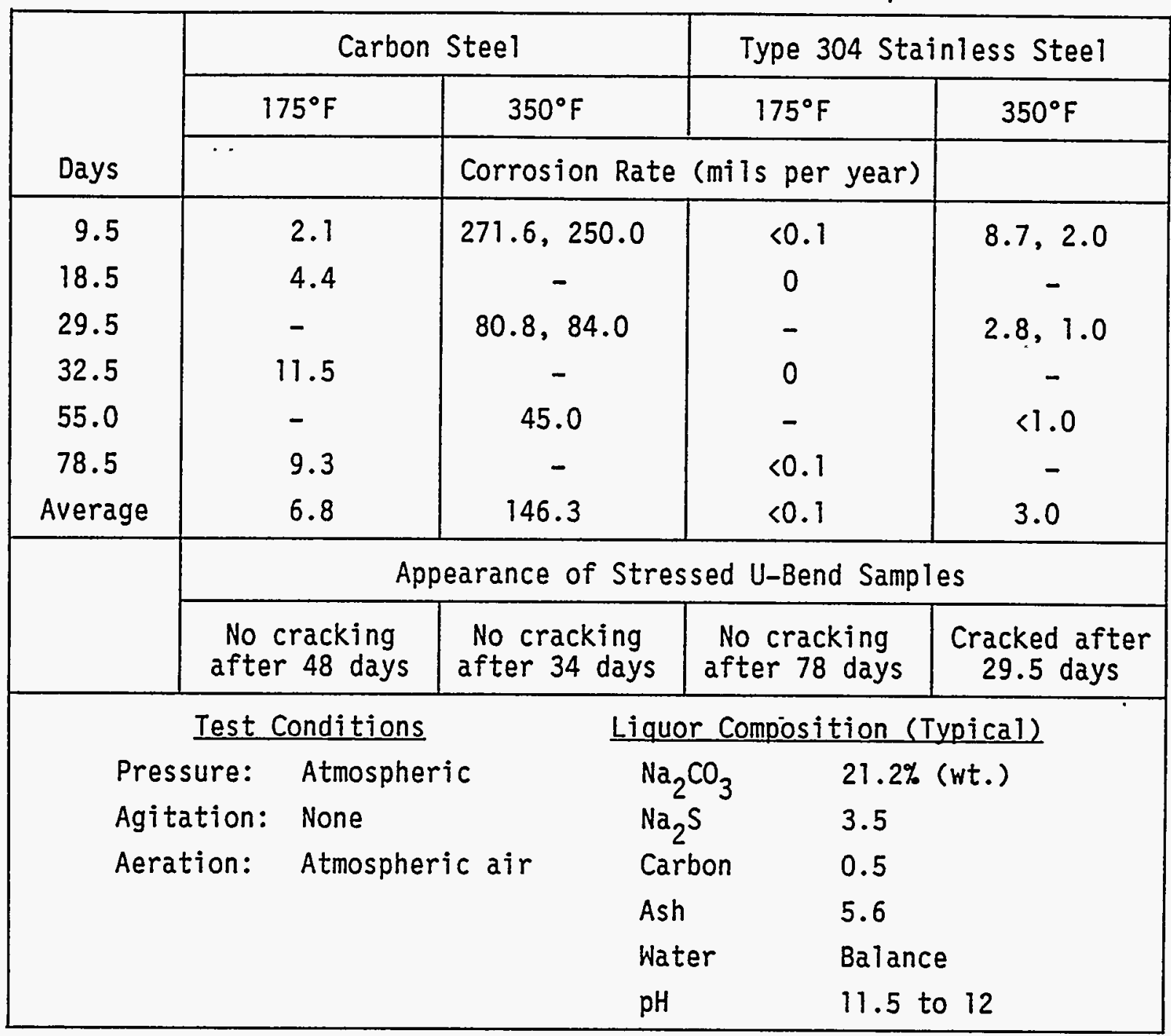

*Data of Reference 8 .

The results indicated a maximum corrosion rate of $26 \mathrm{mils}$ per year (MPY) for carbon steel at $220^{\circ} \mathrm{F}$ and less than 1 MPY for Type 304 stainless steel at $220^{\circ} \mathrm{F}$.

The currently planned pilot plant program includes five runs. If it is assumed that high-temperature green liquor is in the system for 7 days during each run, the total hours of exposure will be $5 \times 7 \times 24=840 \mathrm{~h}$. Using a factor of safety of 10 on the exposure time (i.e., 8,400 h) and the maximum indicated corrosion rate of $26 \mathrm{MPY}$, the total indicated depth of corrosion is 0.025 in. 


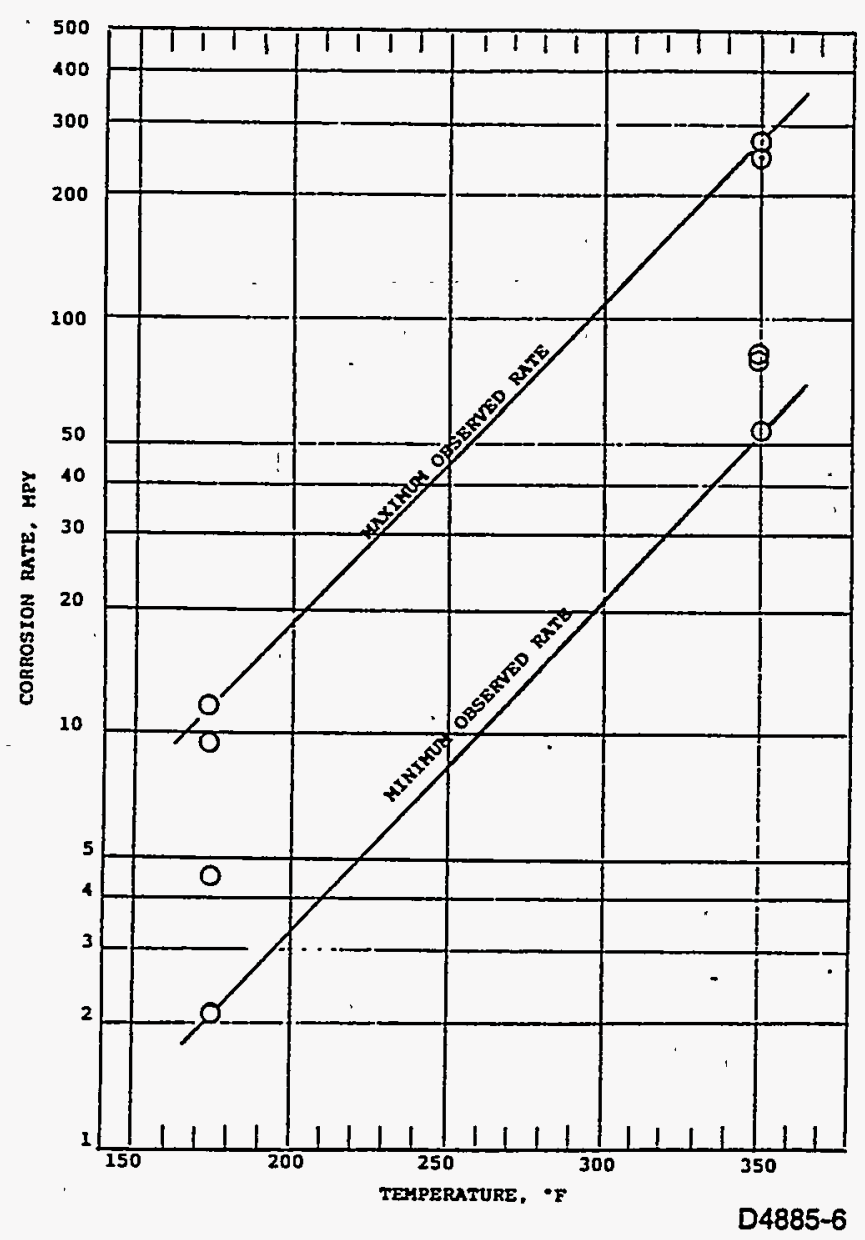

Figure 11. Corrosion Rate of Carbon Steel in Green Liquor

This is an acceptable amount of corrosion for the pilot plant equipment, and carbon steel has, therefore, been selected as the material of construction for the quench tank and vent gas separator vessels. It is recommended that a corrosion allowance of at least $1 / 8 \mathrm{in}$. be added to the wall thickness. To minimize corrosion between runs, it is also recommended that the vessels be drained and filled with inert gas, or preferably drained, rinsed, and thoroughly dried after each run and that the wall thickness be monitored periodically during the period of test operations by appropriate means.

Stainless steel is probably an acceptable alternative to carbon steel, but additional data are needed to prove that it is not susceptible to stress corrosion cracking in green liquor at $220^{\circ} \mathrm{F}$. 


\section{Equipment Arrangement}

The pilot plant will be located in and adjacent to Building 005 at Rockwell International's Santa Susana. Field Laboratory. The general arrangement of equipment is shown in Figures 12 and 13 (drawings M005-68964-M1 and M00568964-M2) representing $p 1$ an and elevation views, respectively.

The gasifier will replace an existing refractory-lined vessel in the high bay of Building 005 and use the same support structure and platforms to the extent feasible. The quench tank will be located in a new pit to be excavated in the floor of the building adjacent to the gasifier support structure.

Product gas and vent gas separators will be located inside the high bay close to the gasifier and quench tank vessels, respectively. The existing feed air heater and the control room with its data acquisition and control (DAC) equipment are also inside Building 005. All other major facility and equipment items are located outside.

Gas leaving the product gas separator at temperatures up to about $1100^{\circ} \mathrm{F}$ flows into an 8-in. stainless steel pipe, which passes through a penetration in the building wall en route to the pressure control valve. Approximately $50 \mathrm{ft}$ of uninsulated stainless steel line is provided to cool the gas to $800^{\circ} \mathrm{F}$ or less before it enters the control valve to minimize problems in procuring and operating this valve. The high-temperature, high-pressure stainless steel line is supported on the outside wall of the building at an elevation of about $20 \mathrm{ft}$ above grade.

The gas pressure is reduced from as high as $901 \mathrm{~b} / \mathrm{in}^{2}$ (absolute) to approximately atmospheric in the pressure control valve. After passing through the valve, the gas flows through low-pressure carbon steel pipe and passes through the product gas cooler, baghouse, and product gas combustor in series. 
As shown in the elevation drawing, the gas cooler consists simply of six vertical passes of 8-in. carbon steel pipe, totaling about $100 \mathrm{ft}$ in length, which will lose heat by convection and radiation to the atmosphere. Control is provided by a bypass line and valve.

The baghouse will be installed on an existing structural support system about $11 \mathrm{ft}$ above grade. This will permit collected dust to be discharged readily into a drum or other vessel. The product gas combustor (incinerator) and its blower are currently in place at the location shown. The combustor installation is complete with regard to controls and utilities; however, refurbishment will be required prior to use for product gas combustion.

The black liquor feed system and green liquor handling system are not shown on the plan and elevation drawings because the optimum location for the required tankage has not yet been established.

\section{PIPING}

Table 6 presents a preliminary list of the main pipe lines in the black liquor gasification pilot plant. The line numbers are identified in the process flow diagram, Figure 1 .

Pipe diameters given in Table 6 are based on a consideration of published correlations for economic pipe diameters ${ }^{9}$ adjusted to take into account special requirements of the pilot plant. Thus, a 2-in. pipe is selected for the black liquor recirculation loop to give a flow velocity of about $10 \mathrm{ft} / \mathrm{s}$ and provide good mixing while 3/4-in. pipe (or 3/4-in. ID tubing) is specified for the black liquor feed line to avoid plugging problems although the resulting flow velocity is only about $2 \mathrm{ft} / \mathrm{s}$. Green liquor and water pipe sizes are selected to give flow velocities of about $7 \mathrm{ft} / \mathrm{s}$; while pipes carrying gases are sized to give maximum velocities ranging from about $50 \mathrm{ft} / \mathrm{s}$ for highpressure gas to about $130 \mathrm{ft} / \mathrm{s}$ for low-pressure gas. 


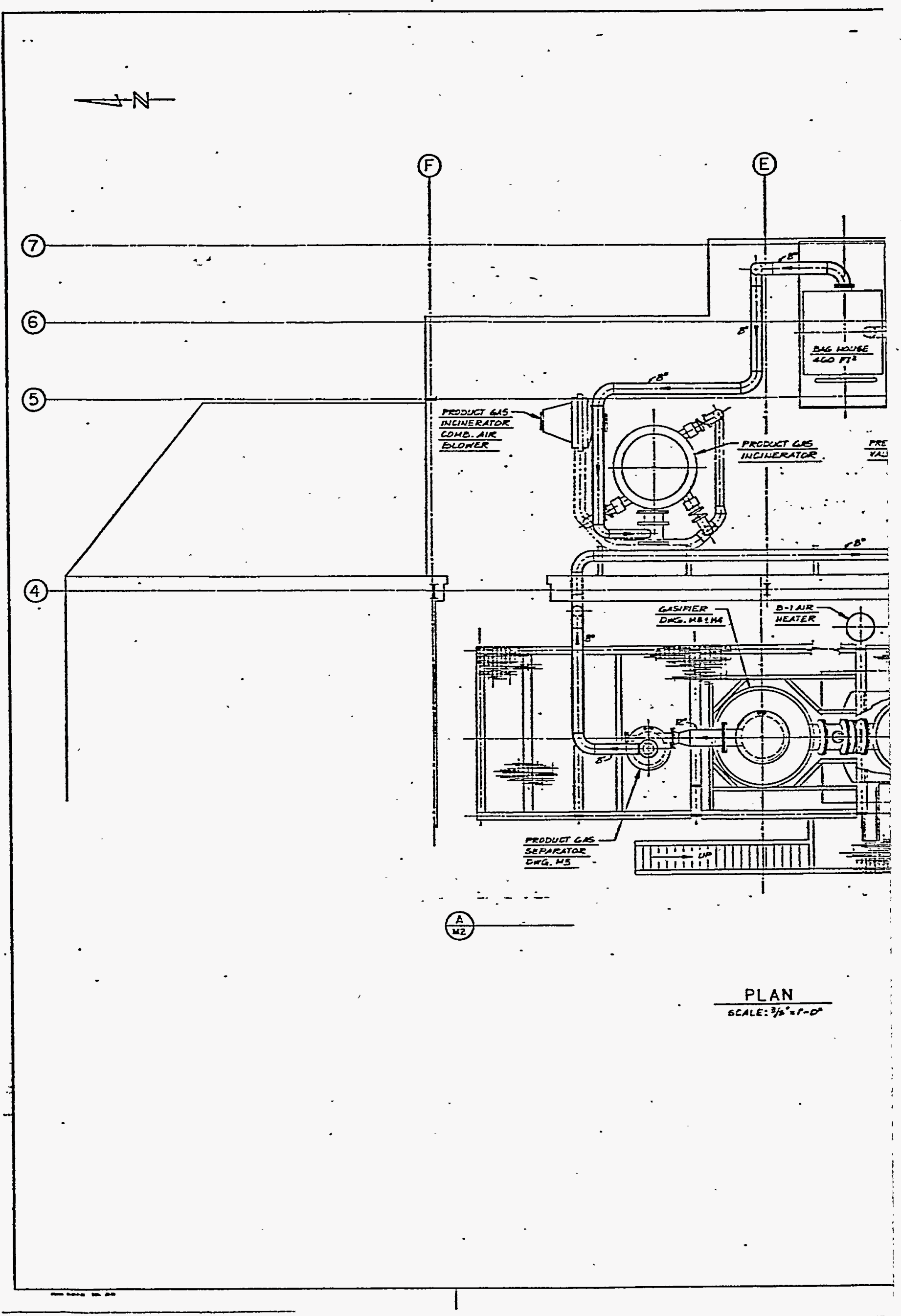


(D)

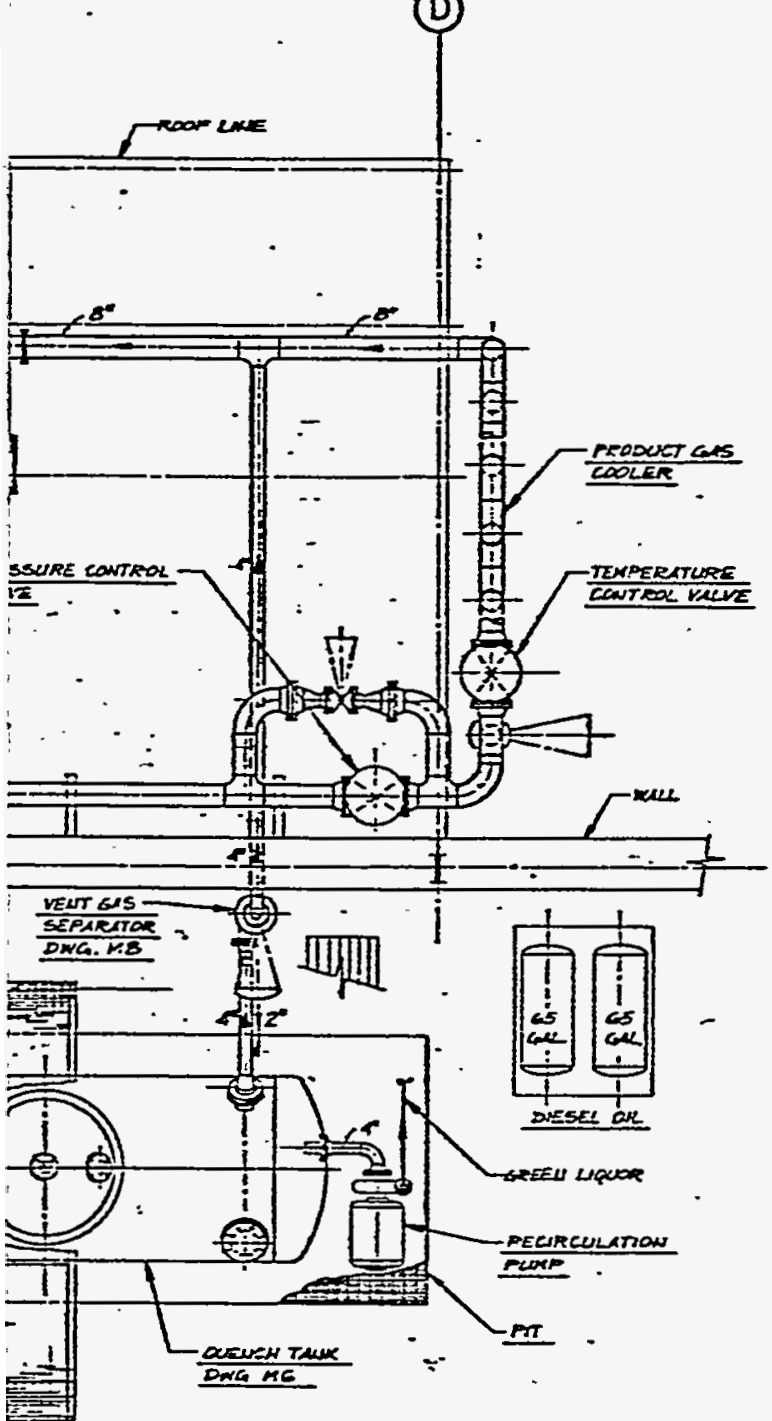




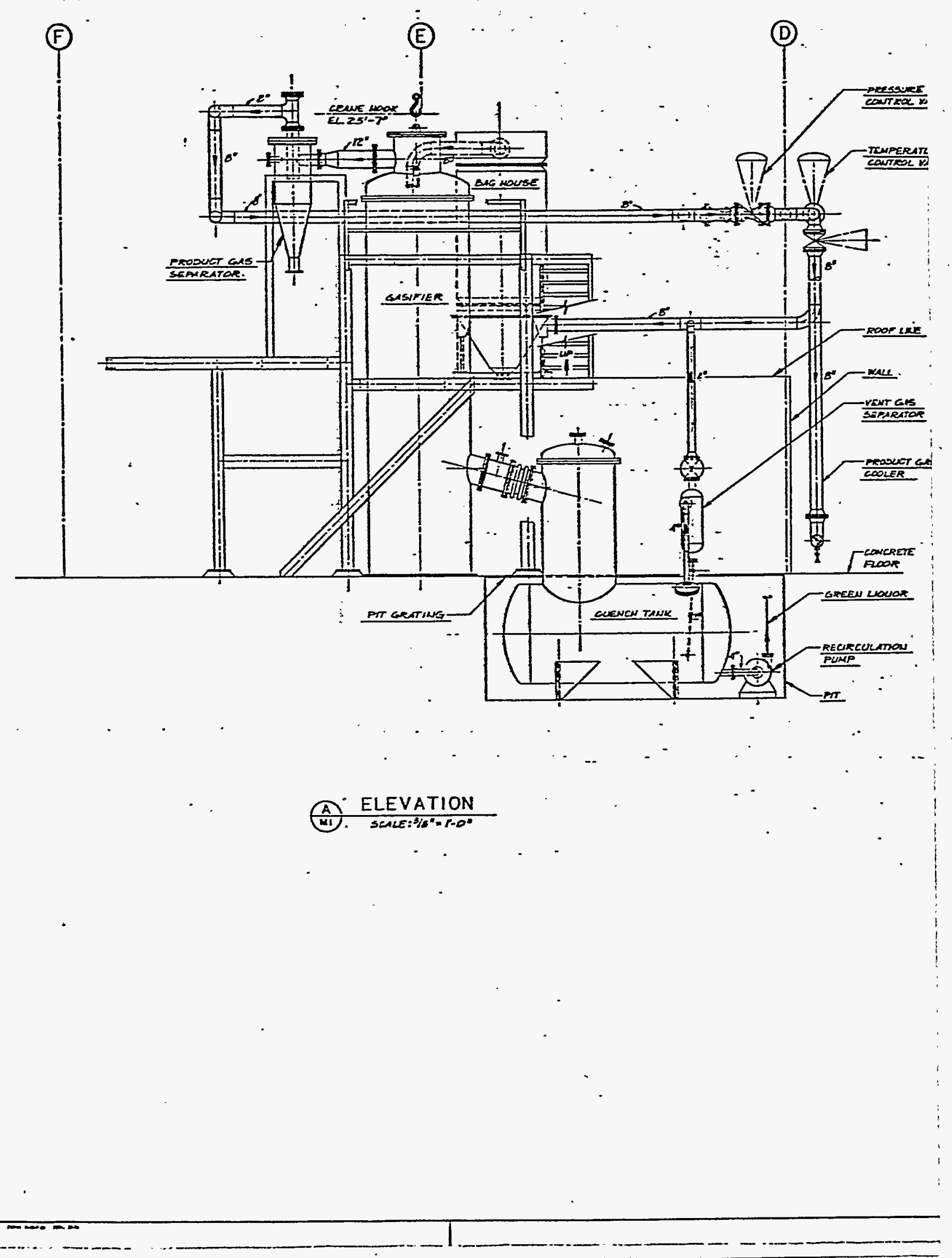




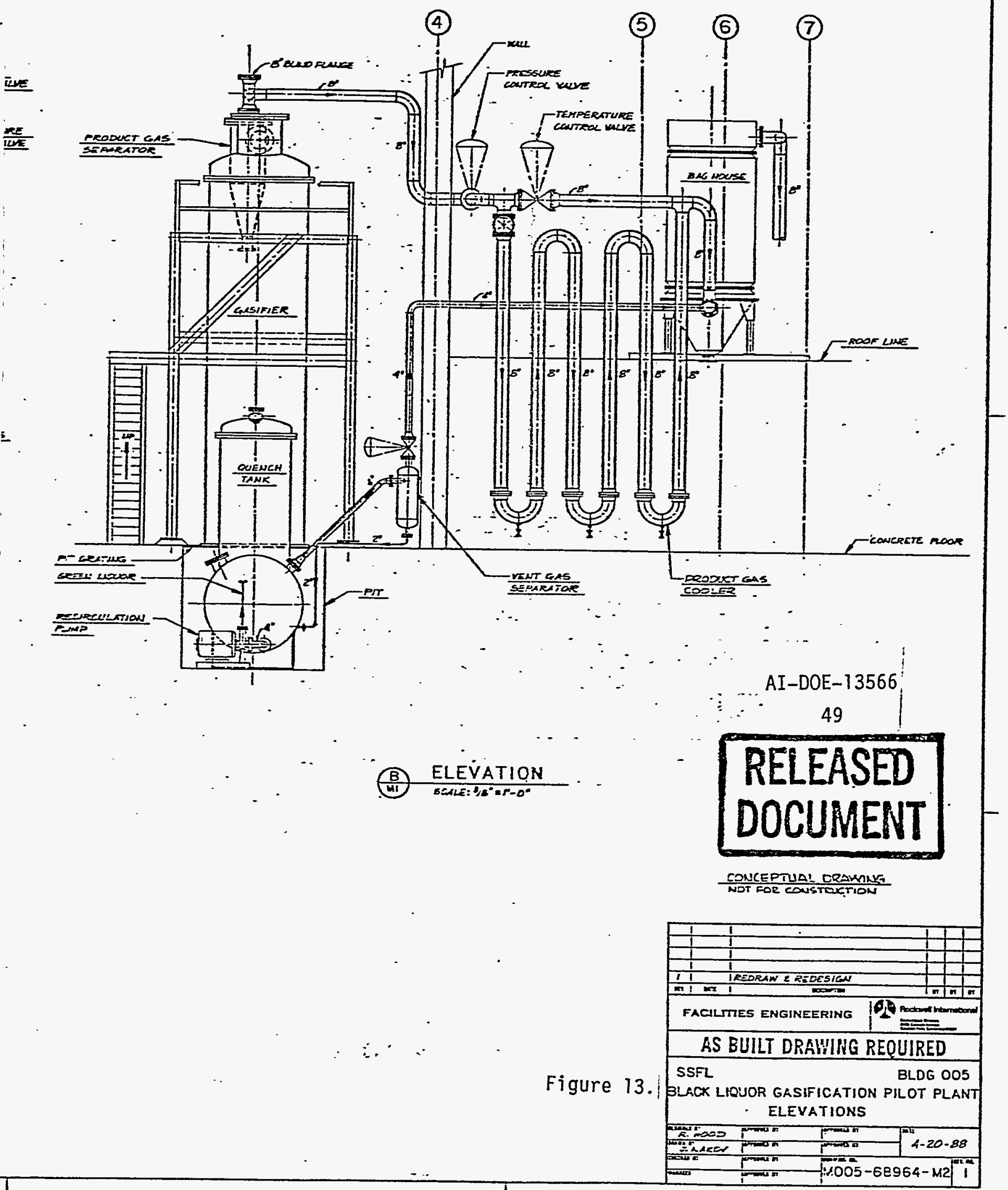


Table 6. Pipeline List

(Sheet 1 of 2)

\begin{tabular}{|c|c|c|c|c|c|c|c|c|c|c|c|}
\hline \multirow[b]{2}{*}{$\begin{array}{l}\text { Line } \\
\text { No. }\end{array}$} & \multicolumn{3}{|c|}{ Operating Conditions } & \multicolumn{3}{|c|}{ Flow Rate } & \multicolumn{2}{|c|}{ Extent of Line } & \multicolumn{3}{|c|}{ Line Description } \\
\hline & Fluid & $\begin{array}{l}\text { Pressure } \\
\left(1 \mathrm{~b} / \mathrm{in}^{2}\right. \\
\text { [gage] })\end{array}$ & $\begin{array}{c}\text { Temperature } \\
\left({ }^{\circ} \mathrm{F}\right)\end{array}$ & $1 \mathrm{~b} / \mathrm{h}$ & $\mathrm{gal} / \mathrm{min}$ & $a c f m$ & From & To & $\begin{array}{l}\text { Size } \\
\text { (in.) }\end{array}$ & $\begin{array}{l}\text { Schédule } \\
\text { or psi }\end{array}$ & Material \\
\hline
\end{tabular}

\section{System 100 - Gasifier}

101 Air

102 Prod gas

103 Prod gas

Gas/melt

105 Air

106 Air

$10 B \quad A, B)$ Air

109 Prod gas

$110 \quad$ Prod gas

12 Nat gas

$112(\mathrm{~A}-\mathrm{H})$ Air

System $200-$ Quench Tank

$\begin{array}{ll}201 & \text { Burner gas } \\ 202 & \text { Gas \& melt } \\ 203 & \text { Water } \\ 204 & \text { GL } \\ 205 & \text { GL } \\ 206 & \text { GL } \\ 207 & \text { GL } \\ 208 & \text { Vent gas } \\ 209 & \text { Steam } \\ 210 & \text { GL } \\ 211 & \text { GL } \\ 212 & \text { GL }\end{array}$

$\begin{array}{rc}76 & 500^{\mathrm{b}} \\ 76 & 500^{\mathrm{b}} \\ 136 & 60 \\ 136 & 220 \\ 136 & 220 \\ 6 & 220 \\ 76 & 220 \\ 61 & 220 \\ 150 & 366 \\ 136 & 220 \\ 6 & 220 \\ \sim 10 & 220\end{array}$

85
920
3,228
46,600
9,986
3,819
60,405
379
50
13,805
20,000
$\sim 50,000$

2,674
3,822
4,201
920
2,674
$\sim 1,500$
$\sim 1,500$
$\sim 1,240$
3,822
4,201
6.3
$\sim 400$

$\begin{array}{cl}208^{\mathrm{a}} & \text { Air heater } \\ 436^{\mathrm{a}} & \text { Gasifier } \\ 1689 & \text { Baghouse } \\ 59^{\mathrm{a}} & \text { Gasifier } \\ 127 & \mathrm{C}-1 \\ \sim 71 & \mathrm{C}-2 \\ 104^{\mathrm{a}} & \text { Flow divider } \\ 121 & \text { Line 105 } \\ 430^{\mathrm{a}} & \text { Separator } \\ 2800 & \text { Pressure control } \\ 0.9 & \text { Gas header } \\ \sim 30 & \text { Line 107 }\end{array}$

Flow divider

$4 \quad$ Sch 80

Separator - see drawing CS \& SS

Combustor

Sch 20

Sch 80

Air heater $\quad 3 \quad$ Sch 40

Line 105

Nozzle lines

Burners

Baghouse

Burner H-3, H-7

Gasifier nozzles

Sch 40

Sch 80

Sch 40

Sch 40

Sch 20

Sch 40

Sch 80

CS

CS

CS

CS

CS

SS

CS

CS

$\sim 50,000$

-
-
6.5
84.0
18.0
6.9
108.8
-
-
24.9
36.0
90.1

$\begin{array}{ll}123.6^{c} & \text { Burner H-3 } \\ 211.6^{c} & \text { Burner port } \\ - & \text { Water header } \\ - & \text { Pump P-2 } \\ - & \text { Line 210 } \\ - & \text { Control valve } \\ - & \text { Quench tank } \\ 131.4 & \text { Control valve } \\ 2.5 & \text { Steam header } \\ - & \text { Line 204 } \\ - & \text { Line 204 } \\ - & \text { Tank V-6 }\end{array}$

Quench tank

Quench tank

Shatter nozzle

Wall wash nozzle

Line 211

Pump P-2

Line 110

Quench tank

Control valve

Tank V-6

Truck connection

Sch 80

Sch 80

$\operatorname{Sch} 40$

Sch 40

$1-1 / 2 \quad \operatorname{Sch} 40$

Sch 40

Sch 40

Sch 40

$3 / 4$ Sch 40

$1-1 / 2 \quad \operatorname{Sch} 40$

$-1 / 2 \quad \operatorname{Sch} 40$

Sch 40

CS
$C S$
$C S$
$C S$
$C S$
$C S$
$C S$
$C S$
$C S$
$C S$
$C S$
$C S$


Table 6. Pipeline List

(Sheet 2 of 2)

\begin{tabular}{|c|c|c|c|c|c|c|c|c|c|c|c|}
\hline \multirow[b]{2}{*}{$\begin{array}{l}\text { Line } \\
\text { No. }\end{array}$} & \multicolumn{3}{|c|}{ Operating Conditions } & \multicolumn{3}{|c|}{ Flow Rate } & \multicolumn{2}{|c|}{ Extent of Line } & \multicolumn{3}{|c|}{ Line Description } \\
\hline & Fluid & $\begin{array}{c}\text { Pressure } \\
\text { (1b/in }{ }^{2} \\
\text { [gage]) }\end{array}$ & $\begin{array}{c}\text { Temperature } \\
\left({ }^{\circ} \mathrm{F}\right)\end{array}$ & $\mathrm{lb} / \mathrm{h}$ & $\mathrm{gat} / \mathrm{min}$ & acfm & From & To & $\begin{array}{l}\text { Size } \\
\text { (in.) }\end{array}$ & $\begin{array}{l}\text { Schedule } \\
\text { gr psi }\end{array}$ & Material \\
\hline \multicolumn{12}{|c|}{ System 200 - Quench Tank (Continued) } \\
\hline $213^{\circ}$ & Wațer & 136 & 60 & $0-3,000$ & $0-5.4$ & - & Line 203 & Separator $v-4$ & $3 / 4$ & $\operatorname{Sch} 40$ & CS \\
\hline 215 & Water & 76 & 220 & $0-3,000$ & $0-5.4$ & - & Separator tank & Quench tank & 2 & $\operatorname{Sch} 40$ & CS \\
\hline 216 & Vent gas & 76 & 220 & 379 & - & $81.7^{\mathrm{C}}$ & Separator V-4 & Control valve & 4 & Sch 40 & CS \\
\hline 217 & Hater & 150 & 60 & $0-50,000$ & $0-100$ & - & Water supply line & Shatter nozzle & 2 & $\operatorname{Sch} 40$ & CS \\
\hline \multicolumn{12}{|c|}{ System $300-$ Black Liguor Feed } \\
\hline 301 & $B L$ & 10 & 220 & 64,985 & 100 & - & BL tank V-5 & Pump P-1 & 3 & $\operatorname{Sch} 40$ & cs \\
\hline 302 & BL & 5 & 220 & 64,594 & 99 & - & End of line 305 & BL. tank V-5 & 2 & $\operatorname{Sch} 40$ & $\operatorname{cs}^{d}$ \\
\hline 303 & BL & 106 & 220 & 2,068 & 3.2 & - & Pump $P-4$ & Gasifier & $3 / 4^{d}$ & $\operatorname{Sch} 40^{d}$ & $c s^{d}$ \\
\hline 305 & BL & 15 & 220 & 64,985 & 100 & - & Pump $P_{-1}$ & Start of line 304 & 2 & Sch 40 & cs \\
\hline 306 & BL. & 0 & 220 & $\sim 10,000$ & $\sim 15.4$ & - & Test tank $v-7$ & Punp $P-3$ & $1-1 / 2$ & $\operatorname{Sch} 40$ & $\mathrm{CS}$ \\
\hline 307 & BL & 10 & 220 & $\sim 10,000$ & $\sim 15.4$ & - & Pump $P-3$ & BL tank V-5 & $i$ & Sch 40 & $\operatorname{cs}$ \\
\hline 308 & $B L$ & 106 & 220 & $0-2,068$ & $0-3.2$ & - & Pump P-4 & Line 302 & $3 / 4^{d}$ & $\operatorname{Sch} 40^{d}$ & $\mathrm{cs}^{\mathrm{d}}$ \\
\hline 309 & BL. & 106 & 220 & 2,068 & 3.2 & - & Line 303 & Test tank $V-7$ & $3 / 4^{d}$ & $\operatorname{sch} 40^{d}$ & $\mathrm{cs}^{d}$ \\
\hline 310 & BL & 15 & 220 & $\sim 50,000$ & $\sim 76.9$ & - & Truck connection & Line 301 & 3 & $\operatorname{Sch} 40$ & CS \\
\hline
\end{tabular}

Notes:

Acfm will be 40 to $50 \%$ higher during operation at $1.0 \mathrm{~atm}$ pressure

Metal pipe temperature (refractory lined)

Acfm given is for 1.0 atm pressure with Burner 3 in operation

304 SS tubing may be substituted (3/4-in. ID)

Abbreviations:

acfm = actual cubic feet per minute

$\mathrm{GL}=$ areen 7 iquor

CS $=$ alack liquor

SS = stainless steel Type 304

1603D/sjh 
All pipe is carbon steel with the exception of elevated pressure lines operating at temperatures above $700^{\circ} \mathrm{F}$, which are Type 304 stainless steel. These are primarily the lines carrying hot product gas from the product gas separator to the pressure control valve. The piping, which conveys the small stream of black liquor feed from the feed pump to the nozzles of the gasifier, is specified to be 3/4-in. carbon steel pipe with the option of using 3/4-in. ID Type 304 stainless steel tubing for this service.

The piping is generally Schedule 40. Exceptions are large size, lowpressure pipes where significant cost and weight savings can be realized by using Schedule 10, and high-temperature/high-pressure applications where the additional strength and rigidity of Schedule 80 pipe is believed to be justified.

Flanged connections will be used on all lines larger than 1 in. and on Schedule 80 l-in. lines. Flanges will be installed at the connections to valves, strainers, pumps, and equipment items and in piping runs, as required, to provide easy installation and disassembly. Welding may be used elsewhere on these lines.

Unless otherwise specified, 150-1b flanges will be used on Schedule 40 pipe and 300-1b flanges on Schedule 80 pipe connections. Schedule 40 carbon steel pipe $1 \mathrm{in}$. and smaller will be assembled using conventional threaded joints. Tubing will be connected using standard compression or bite-type fittings.

Instrument air and sample lines are not included in the pipe line list. These will generally be 1/4-in, Type 304 stainless steel tubing connected with compression fittings and field routed. 


\section{E. INSTRUMENTATION AND CONTROLS}

\section{Design Guidelines}

This section presents the preliminary requirements for designing the instrumentation and control system for the Black Liquor Gasification Pilot Plant. Overall guidelines for the design are as follows:

1. The existing computer data acquisition and control system (DAC) will be used to the maximum extent practical.

2. Control loops will be as simple as possible with the emphasis on operating flexibility rather than fully automatic plant operation.

3. Remote (control room) operation and control will be provided for critical parameters, including black liquor feed rate, air feed rate, make-up water feed rate, quench tank vent gas flow rate, melt shatter steam flow, gasifier pressure, baghouse inlet gas temperature, and quench tank liquid level.

4. Local instrumentation and control systems will be used for secondary parameters and subsystems, including black liquor heaters, green liquor heater and cooler, feed air heater, $0 i 1$ and gas burners, air compressors, and steam generator.

5. Adequate instrumentation, alarms, and controls will be provided to ensure timely recognition of potential problems, permit rapid remedial action when needed, and ensure personnel safety under all foreseeable operating conditions and events.

6. The extent and precision of measurements will be consistent with the R\&D nature of the program and provide adequate data for the calculation of heat and material balances, and the evaluation and correlation of plant performance.

\section{Instrumentation and Control System Description}

A preliminary list of instrumentation requirements generated on the basis of the above guidelines is given in Table 7 . Where no specific function is noted for instrumentation in the table, the measurements will provide data to aid in operation or in the evaluation and correlation of plant performance. 
Table 7. Preliminary List of Instrumentation Requirements

(Sheet 1 of 8 )

\begin{tabular}{|c|c|c|c|c|}
\hline \multirow[b]{2}{*}{ Parameter } & \multicolumn{2}{|c|}{ Operating Limits } & \multirow[b]{2}{*}{ Symbol } & \multirow[b]{2}{*}{ Function } \\
\hline & Normal & $\operatorname{Max}$ & & \\
\hline \multicolumn{5}{|l|}{ Temperature, of } \\
\hline Air from feed air heater & 60 to 800 & 1,000 & TIC & Control power to air heater \\
\hline Air feed to gasifier & 60 to 800 & 1,000 & $\mathrm{TJ}$ & \\
\hline Air feed upper nozzles (4) & 60 to 800 & 1,000 & TJ & \\
\hline Air feed lower nozzles (4) & 60 to 800 & 1,000 & TJA & $\begin{array}{l}\text { Alarm if too high (melt backup) } \\
\text { or too low (no airflow) }\end{array}$ \\
\hline Gasifier interior (9) & 1600 to 1900 & 2,200 & TJ & \\
\hline Gasifier exterior skin (10) & 300 to 600 & 800 & TJ & \\
\hline Product gas outlet & 800 to 1200 & 2,000 & TJA & Alarm if too high (no liquid spray) \\
\hline Product gas outlet & 800 to 1200 & 2,000 & TI & Backup indicator in control room \\
\hline Product gas separator outlet & 700 to 1100 & 1,200 & TJ & \\
\hline Product gas at control valve & 500 to 800 & 1,000 & TJ & \\
\hline Product gas cooler outlet & 400 to 500 & 500 & $T J$ & \\
\hline Gas to baghouse & 400 to 500 & 500 & $T J C$ & Control cooler bypass valve. \\
\hline Gas to combustor & 300 to 400 & 500 & $\mathrm{TJ}$ & \\
\hline Combustor stack & 1000 to 2000 & 2,200 & TJA & Alarm if too high \\
\hline Ambient air high bay & 60 to 100 & 110 & $T J$ & \\
\hline Melt discharge at gasifier & 1600 to 1900 & 2,200 & $T J$ & \\
\hline Natural Gas preheat burner & 1600 to 1900 & 2,500 & $T 3$ & \\
\hline $0 i 1$ preheat burner tip & 200 to 400 & 500 & $T J$ & \\
\hline Melt line burner & 1200 to 1900 & 2,500 & $\mathrm{TJ}$ & \\
\hline Melt discharge at Q.T. & 1600 to 1900 & 2,000 & $\mathrm{TJ}$ & \\
\hline G.L. in quench tank & 60 to 250 & 250 & TIC & Control power to G.L. heater \\
\hline
\end{tabular}


Table 7. Preliminary List of Instrumentation Requirements (Sheet 2 of 8 )

\begin{tabular}{|c|c|c|c|c|}
\hline \multirow[b]{2}{*}{ Parameter } & \multicolumn{2}{|c|}{ Operating Limits } & \multirow[b]{2}{*}{ Symbol } & \multirow[b]{2}{*}{ Function } \\
\hline & Normal & $\operatorname{Max}$ & & \\
\hline \multicolumn{5}{|l|}{ Temperature, ${ }^{\circ} \mathrm{F}$} \\
\hline G.L. in quench tank & 60 to 250 & 250 & TIC & Control valve on C.W. to G.L. cooler \\
\hline G.L. from G.L. cooler & 60 to 250 & 250 & $T J$ & \\
\hline G.L. to shatter jet & 60 to 250 & 250 & $T J$ & \\
\hline G.L. from Q.T. & 60 to 250 & 250 & TJ & \\
\hline Vent gas from Q.T. & 60 to 500 & 500 & $T J$ & \\
\hline Vent gas from separator & 60 to 250 & 250 & T3 & \\
\hline Cooling water feed & 50 to 100 & 100 & $T J$ & \\
\hline Steam feed & 250 to 350 & 375 & $T \mathrm{~J}$ & 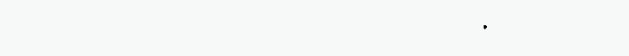 \\
\hline B.L. at tank truck & 100 to 250 & 250 & $\mathrm{TI}$ & Local thermometer \\
\hline B.L. in B.L. tank & 100 to 250 & 250 & TIC & Control power to B.L. tank heater \\
\hline B.L. in B.L. tank & 100 to 250 & 250 & $\mathrm{TJ}$ & \\
\hline B.L. after recirc. pump & 100 to 250 & 250 & $T J$ & \\
\hline B.L. after recirc. heater & 100 to 250 & 250 & TIC & Control power to $B . L$. recirc. heater \\
\hline B.L. after recirc. heater & 100 to 250 & 250. & TJ & \\
\hline B.L. feed stream & 100 to 250 & 250 & TJ & \\
\hline Cooling water return & 50 to 150 & 200 & TJ & \\
\hline \multicolumn{5}{|l|}{ Pressure, $1 \mathrm{~b} / \mathrm{in}^{2}$ (gage) } \\
\hline Air from main compressor & 0 to 120 & 150 & $\mathrm{PJ}$ & \\
\hline Air from backup compressor & 0 to 120 & 150 & PJ & \\
\hline Air to gasifier & 0 to 120 & 150 & PJ & \\
\hline Air to gasifier & 0 to 120 & 150 & PI & Local gauge \\
\hline
\end{tabular}


Table 7. Preliminary List of Instrumentation Requirements (Sheet 3 of 8 )

\begin{tabular}{|c|c|c|c|c|}
\hline \multirow[b]{2}{*}{ Parameter } & \multicolumn{2}{|c|}{ Operating Limits } & \multirow[b]{2}{*}{ Symbol } & \multirow[b]{2}{*}{ Function } \\
\hline & Normal & $\operatorname{Max}$ & & \\
\hline \multicolumn{5}{|l|}{ Pressure, $1 \mathrm{~b} / \mathrm{in}^{2}$ (gage) } \\
\hline Gasifier & 0 to 76 & 80 & PJA & Alarm on high pressure \\
\hline Gasifier & 0 to 76 & 80 & PI & Backup indicator in control room \\
\hline Product gas separator & 0 to 76 & 80 & PJ & \\
\hline Product gas from separator & 0 to 76 & 80 & PJ & \\
\hline Product gas to PCV & 0 to 76 & 80 & PJC & Actuate pressure control valve \\
\hline Product gas to PCV & 0 to 76 & 80 & PIC & $\begin{array}{l}\text { Backup indicator-controller in } \\
\text { control room }\end{array}$ \\
\hline Product gas to $\mathrm{PCV}$ & 0 to 76 & 80 & PI & Local gauge \\
\hline Product gas from PCV & 0 to 2 & 5 & PJ & \\
\hline Product gas from PCV & 0 to 2 & 5 & PI & Local gauge \\
\hline $\begin{array}{l}\text { Product gas to baghouse, } \\
\text { in. } \mathrm{H}_{2} \mathrm{O}\end{array}$ & 0 to 15 & 20 & PJ & \\
\hline $\begin{array}{l}\text { Product gas from baghouse, } \\
\text { in. } \mathrm{H}_{2} \mathrm{O}\end{array}$ & 0 to 5 & 10 & PJ & $\cdot$ \\
\hline Natural gas supply & 0 to 30 & 45 & PI & Local gauge \\
\hline Quench tank & 0 to 76 & 80 & $\mathrm{PJ}$ & \\
\hline Quench tank & 0 to 76 & $80^{\circ}$ & PI & Local gauge \\
\hline Green liquor recirc. pump in & 0 to 76 & 80 & PJ & \\
\hline Green liquor recir. pump out & 40 to 116 & 150 & PJ & \\
\hline $\begin{array}{l}\text { Vent gas after control valve, } \\
\text { in. } \mathrm{H}_{2} \mathrm{O}\end{array}$ & 0 to 20 & 25 & PJ & \\
\hline B.L. intake line & 0 to 15 & 20 & PI & Local gauge \\
\hline B.L. $\operatorname{tank}$ & 0 to 15 & 20 & PI & Local gauge \\
\hline B.L. recirc. pump inlet & 0 to 15 & 20 & PJ & \\
\hline
\end{tabular}


Table 7. Preliminary List of Instrumentation Requirements

(Sheet 4 of 8 )

\begin{tabular}{|c|c|c|c|c|}
\hline \multirow[b]{2}{*}{ Parameter } & \multicolumn{2}{|c|}{ Operating Limits } & \multirow[b]{2}{*}{ Symbol } & \multirow[b]{2}{*}{ Function } \\
\hline & Normal & $\operatorname{Max}$ & & \\
\hline \multicolumn{5}{|l|}{ Pressure, $1 \mathrm{~b} / \mathrm{in}^{2}$ (gage) } \\
\hline B.L. recirc. pump outlet & 0 to 30 & 30 & PJ & \\
\hline B.L. feed pump inlet & 0 to 15 & 20 & PJ & \\
\hline B.L. feed pump outlet & 20 to 116 & 150 & PJ & \\
\hline B.L. feed nozzle, gasifier & 20 to 116 & 150 & $\mathrm{PJ}$ & \\
\hline B.L. feed nozzle, test tank & 0 to 50 & 60 & PI & Local gauge \\
\hline Test tank & 0 to 15 & 15 & PI & Local gauge \\
\hline Process water supply & 0 to 150 & 200 & PI & Local gauge \\
\hline Makeup water supply & 0 to 100 & 150 & PI & Local gauge \\
\hline Nitrogen gas supply & 0 to 1000 & 2000 & PI & Local gauge \\
\hline \multicolumn{5}{|l|}{ Differential Pressure } \\
\hline $\begin{array}{l}\text { Gasifier outlet/separator, } \\
\text { psi }\end{array}$ & 0 to 1 & 2 & DPJA & Alarm on high $\Delta P$ (plugged outlet) \\
\hline $\begin{array}{l}\text { Baghouse outlet/separator, } \\
\text { in. } \mathrm{H}_{2} \mathrm{O}\end{array}$ & 0 to 10 & 20 & DPI & Local DP gauge \\
\hline G.L. product strainer, $1 \mathrm{~b} / \mathrm{in}^{2}$ & 0 to 5 & 10 & DPI & Local OP gauge \\
\hline B.L. loop strainer, $1 \mathrm{~b} / \mathrm{in}^{2}$ & 0 to 5 & 10 & DPI & Local DP gauge \\
\hline B.L. feed strainer, $1 b / i n^{2}$ & 0 to 5 & 10 & DPI & Local DP gauge \\
\hline \multicolumn{5}{|l|}{ Flow Rate } \\
\hline Total air to gasifier, $1 \mathrm{~b} / \mathrm{h}$ & 0 to 2700 & 3,000 & $\mathrm{FJC}$ & Control air feed valve \\
\hline Total air to gasifier, $1 \mathrm{~b} / \mathrm{h}$ & 0 to 2700 & 3,000 & FIC & Backup indicator-controller \\
\hline Feed air to heater, $1 \mathrm{~b} / \mathrm{h}$ & 0 to 2700 & 3,000 & FJ & \\
\hline $\begin{array}{l}\text { Feed air to lower nozzle, } \\
\text { lb/h }\end{array}$ & 0 to 1800 & 3,000 & FJ & \\
\hline $\begin{array}{l}\text { Feed air to upper nozzle, } \\
\text { lb/h }\end{array}$ & 0 to 1800 & 3,000 & FJ & \\
\hline
\end{tabular}


Table 7. Preliminary List of Instrumentation Requirements (Sheet 5 of 8 )

\begin{tabular}{|c|c|c|c|c|c|}
\hline \multirow[b]{2}{*}{ Parameter } & \multicolumn{3}{|c|}{ Operating Limits } & \multirow[b]{2}{*}{ Symbol } & \multirow[b]{2}{*}{ Function } \\
\hline & & Normal & $\operatorname{Max}$ & & \\
\hline \multicolumn{6}{|l|}{ Flow Rate } \\
\hline G.L. recycle, gal/min & 0 & to 100 & 125 & FJA & Alarm on low flow to shatter nozzle \\
\hline G.L. wall wash, gal/min & 0 & to 25 & 35 & FJA & Alarm on low flow to wall wash \\
\hline G.L. product, gal/min & 0 & to 20 & 30 & FJ & \\
\hline G.L. product, gal & 0 & to 50,000 & 100,000 & FQI & $\begin{array}{l}\text { Local, measures total quantity } \\
\text { produced }\end{array}$ \\
\hline Vent gas, $1 \mathrm{~b} / \mathrm{h}$ & 0 & to 500 & 800 & FJC & Control vent gas valve \\
\hline Makeup water, gal & 0 & to 50,000 & 100,000 & FQI & Local, measures total water added \\
\hline Vent gas wash water, gai/min & 0 & to 5 & 10 & FI & Local rotometer \\
\hline Shatter steam, $1 \mathrm{~b} / \mathrm{h}$ & 0 & to 50 & 75 & $\mathrm{FJC}$ & Control shatter steam valve \\
\hline Black liquor recirc., gal/min & & to 100 & 125 & FJ & Mass flow meter \\
\hline Black liquor feed, lbih & 0 & to 2500 & 3,000 & FJC & Mass flow meter, feed valve control \\
\hline Black liquor feed, $1 \mathrm{~b} / \mathrm{h}$ & 0 & to 2500 & 3,000 & FIC & Backup indicator-controller \\
\hline Black liquor feed, gal/min & 0 & to 3.5 & 4.0 & $\mathrm{FJC}$ & Volumetric flow, alternate control \\
\hline 0il to preheat burner, $1 \mathrm{~b} / \mathrm{h}$ & 0 & to 60 & 100 & FJ & \\
\hline Air to oil burner, $1 \mathrm{~b} / \mathrm{h}$ & 0 & to 1000 & 7,500 & FJ & \\
\hline $\begin{array}{l}\text { Sweep air to oil burner, } \\
l b / h \text {. }\end{array}$ & 0 & to 1000 & 1,500 & FJ & \\
\hline $\begin{array}{l}\text { Natural gas to preheat } \\
\text { burner, SCFH }\end{array}$ & 0 & to 75 & 100 & FI & Local flow meter \\
\hline $\begin{array}{l}\text { Natural gas to melt disch. } \\
\text { burner, SCFH }\end{array}$ & 0 & to 75 & 100 & FI & Local flow meter \\
\hline $\begin{array}{l}\text { Air to N.G. preheat burner, } \\
\text { lb/h }\end{array}$ & & to 100 & 150 & FJ & \\
\hline
\end{tabular}


Table 7. Preliminary List of Instrumentation Requirements (Sheet 6 of 8 )

\begin{tabular}{|c|c|c|c|c|}
\hline \multirow[b]{2}{*}{ Parameter } & \multicolumn{2}{|c|}{ Operating Limits } & \multirow[b]{2}{*}{ Symbol } & \multirow[b]{2}{*}{ Function } \\
\hline & Normal & $\operatorname{Max}$ & & \\
\hline \multicolumn{5}{|l|}{ Flow Rate } \\
\hline $\begin{array}{l}\text { Air to melt disch. burner } \\
1 \mathrm{~b} / \mathrm{h}\end{array}$ & 0 to 100 & 150 & FJ & \\
\hline $\begin{array}{l}\text { N.G. to product gas } \\
\text { combustor, SCFH }\end{array}$ & 0 to 100 & 150 & FI & Local flow meter \\
\hline \multicolumn{5}{|l|}{ Liquid Level } \\
\hline Quench tank level, ft & 3.0 & $1.0,6.0$ & LJC & Control product G.L. valve \\
\hline Quench tank level alarm, ft & 3.0 & $2.5,4.0$ & L.JA & High- and low-level alarms \\
\hline G.L. storage tank level, $\%$ & 0 to 75 & 80 & L.JA & High-level alarm \\
\hline B.L. tank level, $\%$ & 0 to 75 & 80 & $L I, L J$ & Local level gauge and DAC \\
\hline $\begin{array}{l}\text { Spray nozzle test tank } \\
\text { level, } \%\end{array}$ & 0 to 75 & 80 & LI & Local level gauge \\
\hline Vent gas separator level, $\%$ & 0 & 50 & $L I, L J A$ & $\begin{array}{l}\text { Local level gauge and high-level } \\
\text { alarm }\end{array}$ \\
\hline \multicolumn{5}{|l|}{ Densitometers } \\
\hline Black liquor feed, g/cc & 1.20 to 1.35 & 1.40 & DMJ & \\
\hline Green liquor product, $g / c c$ & 1.00 to 1.15 & $1.20^{\circ}$ & DMJ & \\
\hline \multicolumn{5}{|l|}{ Weigh Scales } \\
\hline B.L. tank weight, ib & 0 to 80,000 & 95,000 & WJ & 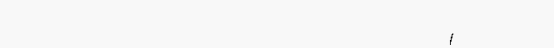 \\
\hline $\begin{array}{l}\text { Spray nozzle test tank } \\
\text { weight, lb }\end{array}$ & 0 to 5,000 & 6,000 & WJ & \\
\hline $0 i 1$ tank weight, $1 \mathrm{~b}$ & 0 to 1,000 & 1,000 & WJ & \\
\hline
\end{tabular}


Table 7. Preliminary List of Instrumentation Requirements (Sheet 7 of 8 )

\begin{tabular}{|c|c|c|c|c|}
\hline \multirow[b]{2}{*}{ Parameter } & \multicolumn{2}{|c|}{ Operating Limits } & \multirow[b]{2}{*}{ Symbol } & \multirow[b]{2}{*}{ Function } \\
\hline & Normal & $\operatorname{Max}$ & & \\
\hline \multicolumn{5}{|l|}{ I\&C Package Systems } \\
\hline $\begin{array}{l}\text { N.G. preheat burner, flame } \\
\text { safety and control system }\end{array}$ & -- & - & $\mathrm{BCS}$ & \\
\hline $\begin{array}{l}\text { 0il preheat burner, flame } \\
\text { safety and control system }\end{array}$ & -- & - & BCS & \\
\hline $\begin{array}{l}\text { Melt discharge burner, flame } \\
\text { safety and control'system }\end{array}$ & - & - & BCS & \\
\hline $\begin{array}{l}\text { Product gas combustor, flame } \\
\text { safety and control system }\end{array}$ & -- & - & $\mathrm{BCS}$ & \\
\hline $\begin{array}{l}\text { Main air compressor control } \\
\text { system }\end{array}$ & - & - & $\operatorname{ccs}$ & \\
\hline $\begin{array}{l}\text { Backup air compressor control } \\
\text { system }\end{array}$ & - & - & $\operatorname{ccs}$ & \\
\hline $\begin{array}{l}\text { Steam generator control } \\
\text { system }\end{array}$ & - & -- & SGCS & \\
\hline
\end{tabular}


Table 7. Preliminary List of Instrumentation Requirements (Sheet 8 of 8 )

\begin{tabular}{|c|c|c|c|c|}
\hline & Inst & t Symbols & Abbreviations & \\
\hline & DAC & Data Acquisition and Control Center & B.L. = Black Liquor & \\
\hline & TIC & Temperature Indicator Controller & G.L. $=$ Green Liquor & \\
\hline & TJ & Temperature to DAC & Q.T. = Quench Tank & \\
\hline & TJA & Temperature to DAC with Alarm & C.W. = Cooling Water & : \\
\hline & TJC & Temperature to DAC with Control & N.G. = Natural Gas & \\
\hline & TI & Temperature Indicator & & \\
\hline & PJ & Pressure to DAC & & \\
\hline & PJA & Pressure to DAC with Alarm & & \\
\hline & PJC & Pressure to DAC with Control & & \\
\hline & PIC & Pressure Indicator Controller & & \\
\hline & PI & Pressure Indicator & & \\
\hline & PCV & Pressure Control Valve & & \\
\hline & DPJ & Differential Pressure to DAC & & \\
\hline$\mapsto$ & DPJA & Differential Pressure to DAC with Alarm & & \\
\hline ○్ & DPI & Differential Pressure Indicator & & \\
\hline N I & FJ & Flow Rate to DAC & & \\
\hline$\vec{\omega}$ & FI & Flow Rate Indicator & & \\
\hline G & FJC & Flow Rate to DAC with Control & & \\
\hline & FIC & Flow Rate Controller & & \\
\hline & FQI & Flow Totalizer, Local Indicator & & \\
\hline & LI & Level Indicator & & \\
\hline & LJ & Level to DAC & & \\
\hline & LJC & Level to DAC with Control & & \\
\hline & LJA & Level to DAC with Alarm & & \\
\hline & WJ & Weigh Scale Reading to DAC & & \\
\hline & BCS & Burner Flame Safety and Control System (Near Burner) & & \\
\hline & $\operatorname{ccs}$ & Compressor Control System (At Compressor) & & \\
\hline & SGCS & Steam Generator Control System (Part of Boiler) & & \\
\hline & DMJ & Densitometer, Signal to DAC & & \\
\hline
\end{tabular}


Operation of the pilot plant, with emphasis on the instrumentation and control approach, is described briefly below. Equipment numbers refer to the process flow diagram (Figure 1) and the equipment list (Table 4).

Black liquor feed is stored in the black liquor tank, V-5. The temperature in this tank is maintained at about $200^{\circ} \mathrm{F}$ by the black liquor tank heater, $\mathrm{H}-4$, which is controlled automatically by a local temperature sensor and controller. Black liquor is continuously circulated from this tank through a pump, $P-1$, a strainer, $S-2$, an in-line heater, $H-5$, and back to the tank. The rate of flow in this loop is measured, but not controlled automatically since it has little effect on plant operation. The heater, $\mathrm{H}-5$, augments the tank heater, $\mathrm{H}-4$, to ensure that the black liquor feed stream is adequately heated. It is locally controlled by a temperature sensor in the black liquor recirculation line. The pressure drop across the strainer is indicated locally to warn the operator of possible plugging. The black liquor recirculation loop serves to mix, heat, and remove large solid particles from the black liquor prior to gasification. The density of black liquor in the loop is continuously monitored by a densitometer which transmits its signal to the DAC.

A small feed stream of black liquor flows from the recirculation loop through a fine strainer, S-3, and black liquor feed pump, $P-4$, which raises its pressure for injection into the gasifier. The black liquor feed rate is a critical parameter and is measured by both a mass flow meter and a volumetric flow meter, both of which provide signals to the control room. Either meter may be used as the primary source of control to actuate the black liquor feed rate control valve. Control is normally accomplished by the DAC; however, a backup indicator-controller is provided in the control room for use in case of a DAC failure. As a further check on the amount of black liquor fed into the system, the black liquor feed tank is equipped with a weigh scale, which provides a signal to the DAC. A local differential pressure gauge across the strainer, $5-3$, is used to provide evidence of plugging. 
Air at a conștant pressure (normally $1201 \mathrm{~b} / \mathrm{in}^{2}$ [gage]) is provided by either or both compressors, $\mathrm{C}-1$ and $\mathrm{C}-2$, which are locally controlled by existing systems. The total rate of airflow to the gasifier is measured and controlled automatically by a control valve actuated from the control room. During normal operation, this function is provided by the DAC; however, a backup indicator-controller is provided. The air and black liquor feed rates will be independently set and adjusted by the operator on the basis of the desired throughput and indicated gasifier temperature. The amount of air entering the heater, $\mathrm{H}-2$, will be metered to provide data on the main stream of feed air, which enters through the upper and lower nozzles. The ratio of air feed to the two nozzle levels will be determined by flow meters in both branches of the air feed line, and adjusted by the manual operation of valves in these branch lines.

The main stream of gasifier feed air is preheated by the heater, $\mathrm{H}-2$, to about $700^{\circ} \mathrm{F}$. The selected preheat temperature is maintained by a local controller, which regulates power to the heater. Temperatures within the gasifier are monitored by nine thermocouples. The readings of key thermocouples are displayed continuously by the DAC for operator information, but are not used for automatic control of feed rates or other parameters. Other key gasifier temperatures that will be monitored by the DAC include the air feed nozzles ( 8$)$, the gasifier vessel outside wall ( 10 places), and the product gas outlet. This key temperature will also be shown on a backup temperature indicator in the control room. The temperatures at the lower air feed nozzles will be set to activate an alarm at either high or low temperatures indicating possible melt backup into the nozzles or airflow interruption, respectively. The product gas temperature will also be set to alarm at high temperature, which would be an early indication of black liquor feed flow stoppage.

Product gas from the gasifier flows to the separator, $V-3$, then through an uninsulated stainless-steel line, where it cools to less than $800^{\circ} \mathrm{F}$ before entering the main pressure control valve. This pressure control valve is set in the control room to hold the gasifier at the desired operating pressure. 
It is normally actuated by the DAC; however, a separate backup pressure indicator-controller is provided. The automatic control valve is augmented by a manual bypass valve that can be opened in the event of plugging of the main valve. As added safety measures, the gasifier vessel is equipped with a pressure relief valve and a high-pressure alarm in the control room. The differential pressure across the product gas outlet port and separator is also moni-. tored, and this measurement is set to alarm at high $\Delta P$, indicating possible plugging in this region.

After passing through the main pressure control valve, the product gas flows at near-atmospheric pressure through the product gas cooler, H-9, baghouse, S-1, and product gas combustor, $\mathrm{H}-1$. The purpose of the product gas cooler is to ensure that the product gas temperature is below the maximum allowable temperature of the cloth bags in the baghouse. Excessive cooling is aiso undesirable since the condensation of water in the baghouse can cause poor flow of powder product and other operating problems. The amount of gas cooling is, therefore, controlled automatically by a temperature sensor in the feed line to the baghouse that is monitored by the DAC and actuates a valve in the cooler bypass line.

Operation of the baghouse is monitored primarily by a differential pressure measurement and by temperature and pressure readings on inlet and outlet streams, all of which are transmitted to the DAC. The product gas combustor is an existing facility completely equipped with local controls on its air supply and flame safety and burner control system. The rate of natural gas flow to the product gas combustor burner will be measured and recorded by the DAC. The stack gas temperature is also transmitted to the DAC, which is set to provide a high-temperature alarm to warn of improper operating conditions in the combustor.

Product melt and about 10\% of the product gas flow out of the gasifier through the melt discharge line to quench tank, $V-2$. This line is equipped with a natural gas burner, $\mathrm{H}-3$, which aids in maintaining the temperature inside the line above the melting point of the flowing melt (about $1550^{\circ} \mathrm{F}$ ). 
Thermocouples in the line sense the temperature and transmit the data to the DAC. If the melt temperature appears to be too low, the operator has several options including ( 1 ) initiating or increasing gas and airflow to burner, $\mathrm{H}-3$, (2) increasing the flow of gasifier product gas through the line, (3) injecting air (without fuel) through burner, $\mathrm{H}-3$, to react with combustible product gas in the line, and (4) increasing the temperature within the gasifier. It is planned that burner, $\mathrm{H}-3$, will be used primarily during low-pressure, lowcapacity operation of the gasifier and will be turned off prior to operation at the full gasifier pressure of $90 \mathrm{lb} / \mathrm{in}^{2}$ (absolute). Melt and gas throughput are high enough at this condition to maintain a suitably high temperature, and this operating procedure eliminates the need for a natural gas compressor on the burner feed. When in use, burner operation will be controlled by a local flame safety and control system, which meets all requirements for unattended operation. The rate of gas flow will be indicated by a local meter, however, the airflow rate will be transmitted to the DAC since total air input to the gasifier system is an important parameter for material balance calculations.

To ensure adequate breakup of melt falling into the quench tank, both steam and recycle green liquor shatter nozzles will be used. The steam flow rate will be measured and automatically controlled by an appropriate meter and control valve operable from the control room. The green liquor flow of about $84 \mathrm{gal} / \mathrm{min}$ will be measured, but not separately controlled, since the flow rate is not important as long as it is adequate to break up the melt stream. The recycle green liquor stream represents a major portion of the output of the green liquor recirculation pump, P-2. An alarm on low flow in the green liquor recirculation line will warn the operator of a blockage or pump failure, in which case he can activate an independent third shatter nozzle using process water from a high-pressure main.

Prior to melt production in the gasifier and during periods of low melt flow, it is necessary to add heat to the green liquor to maintain the desired operating temperature $\left(180\right.$ to $\left.220^{\circ} \mathrm{F}\right)$. This is accomplished by heater, H-6, which is controlled automatically by a local temperature sensor and power 
controller. At full operating capacity and during non-steady-state periods of high melt flow, it is necessary to remove excess heat from the green liquor. A cooler, $\mathrm{H}-8$, is provided for this purposes. Operation of $\mathrm{H}-8$ is controlled automatically by a valve in the cooling water line actuated locally by a temperature signai from a temperature sensor in the green liquor. A temperature signal is also sent to the DAC to show the temperature of the liquor leaving the cooler.

A large coarse-screen strainer inside the quench tank prevents large particles of solidified melt from leaving the quench tank and entering the suction side of $P-2$. Since they are soluble, particles held on the screen will eventually dissolve and clear the openings. A relatively small slip stream of green liquor is continuously removed from the green liquor recirculation loop downstream of $\mathrm{P}-2$ and passed through a finer strainer, $\mathrm{S}-5$, which removes small particles. This strainer is equipped with a differential pressure indicator to alert the operator to possible plugging. A green liquor flow of about $18 \mathrm{gal} / \mathrm{min}$ is returned to the quench tank from a point downstream of strainer, S-5, to provide a continuous wash for the inside surfaces of the quench tank vertical extension. The flow rate in the wall wash line is monitored by the DAC with an alarm to signal low flow.

A densitometer is used to measure the density of green liquor flowing in the line downstream of $S-5$. The densitometer operates through the DAC to control the flow of makeup water into the quench tank. A local flow totalizer will provide a record of the amount of water added. A small amount of water may also be injected into the vent gas separator to cool and wash this gas. The flow of water used for this purpose will be manually set and indicated by a local flow meter. Since this water drains into the quench tank, it represents a portion of the total makeup water.

Net green liquor product is discharged from the system downstream of strainer, S-5, to one of the green liquor storage tanks, $V-6$ (A and $B$ ). The rate of discharge is controlled automatically by a control valve actuated by the liquid level in the quench tank. Since it is important that an adequate

AI-DOE-13566 
liquid level be maintained in the quench tank at all times to provide feed for pump, P-2, and the shatter nozzle, and also that the level not rise to the point where it could interfere with shatter jet operation or contact hot refractory, two separate liquid level instruments are specified for the quench tank. One liquid level detector is independent of the DAC and operates locally to open and close the green liquor discharge valve. The other operates through the DAC to provide a liquid level reading in the control room and both high- and low-level alarms. Each of the green liquor storage tanks is also provided with a level indicator operating through the DAC to alarm on high levels.

Product gas that leaves the gasifier via the melt discharge line and combustion products from burner, $\mathrm{H}-3$, flows into quench tank, $\mathrm{V}-2$, with the product melt and must be continuously vented. The vent gas flows from the quench tank to a small separator, $V-4$, which serves to remove drops of green liquor or other particles which may be entrained. The separator is equipped with a water injection port for the addition of water to cool and wash the gas, if necessary. Excess water, collected green liquor, and condensed steam drain from the separator by gravity to the quench tank. A liquid level sensor with high-level alarm at the DAC is provided to warn of drain line plugging or other possible cause of increasing liquid level in the separator.

To ensure that 10\% (or other desired amount) of the product gas flows through the melt discharge line into the quench tank, it is necessary to control the flow of gas out of the quench tank. For this purpose, a line is provided from separator, V-4 (which operates at the same pressure as the gasifier and quench tank), to a point of low pressure in the product gas line. A flow control valve in this line limits the amount of gas flowing and also reduces its pressure as required. This control valve is actuated by the DAC based on a flow rate sensor located in the low-pressure (high-velocity) portion of the vent line.

Special equipment items used during start-up or intermittently during operation also require instrumentation and controls. The spray nozzle test 
tank, V-7, is used to permit black liquor flow through the feed system just prior to gasifier operation and also to test spray nozzles with hot black liquor feed. It is equipped with a weigh scale with readout at the DAC, a local level indicator, and a pressure gauge.

Two preheat burners are provided to raise the temperature of the gasifier vessel prior to plant operation. The natural gas burner, $\mathrm{H}-7$, will be used for initial heating and as an igniter and pilot burner for the much larger capacity oil burner, H-10. Both burners are equipped with local flame safety and control systems, which meet all requirements for unattended operation. Natural gas to $\mathrm{H}-7$ is metered with a locally mounted flow indicator. Air feed to $\mathrm{H}-7$, as well as burner air and sweep air to the oil burner, $\mathrm{H}-10$, are metered separately and recorded by the DAC. Since air (without fuel) is fed through the burners during normal operation with black liquor, information on the quantity of this air is required for material balance calculations. The amount of oil flowing to $\mathrm{H}-1 \mathrm{O}$, is indicated by a locally mounted flow meter. In addition, the oil tank is mounted on weigh scales with DAC readout to provide a record of fuel oil used.

Product gas chemical analyses will be performed by using the existing instrumentation. These instruments include one gas chromatograph (GC) with nitrogen carrier gas for hydrogen; a second GC with helium carrier gas for carbon dioxide, ethylene, ethane, oxygen plus argon, nitrogen, methane, and carbon monoxide; and separate continuous analyzers for oxygen, carbon dioxide, carbon monoxide, and total sulfur. The continuous analyses will be tied into the DAC to provide data to the plant operator, but will not be used for automatic control of any plant operation. Gas sample stations will be provided to permit the sampling of gas from the gasifier product line before mixing with the vent gas, the vent gas line, and the mixed gas line between the baghouse and the combustor. 
No continuous chemical analyses. of liquid or solid streams will be performed; however, samples will be withdrawn periodically from the black liquor feed line, green liquor product line, baghouse discharge, and product gas separator discharge for laboratory analysis.

$1603 D / j 1 m$ 


\section{SPRAY NOZZLE TESTING}

\section{A. BACKGROUND AND OBJECTIVES}

The black liquor gasifier requires a spray nozzle with unique characteristics. The drops it produces must be large enough to fall through the drying zone without being entrained in the product gas stream, but small enough to dry completely before they enter the gasification zone at the bottom of the vessel. The spray pattern must provide good coverage of the small-diameter gasifier vessel without excessive impingement on the walls. The nozzle orifice must be relatively large and unobstructed to minimize plugging problems. Finally, the unit used in the pilot plant must be scalable to larger sizes.

Splash-plate and hollow-cone nozzles used for feeding black liquor in recovery boilers are unsuitable because their spray patterns are not compatible with a small-diameter cylindrical gasifier configuration. A review of other commercially available nozzles during the previous phase of the program did not locate a design that would meet all the black liquor process requirements. ${ }^{6,7}$ It was concluded that narrow-angle, full-cone nozzles and twofluid (black liquor/steam) nozzles were the most likely of the commercially available nozzles to be adaptable to the gasifier application. A new concept was also proposed aimed specifically at the requirements of the pilot plant. The new concept has been called a splash-tube nozzle.

The objective of this task is to evaluate the full-cone pressure nozzle, two-fluid nozzle, and splash-tube nozzle designs for applicability to the requirements of black liquor gasification by experimentally testing nozzles of each type with water and hot wax.

AI-DOE-13566 


\section{B. METHOD AND APPARATUS}

The technique of using molten materials for measuring droplet size distributions from spray nozzles has been used by many prior investigators. $10,11,12$ It involves the spraying of a molten liquid (typically a wax product), which will form a droplet spray characterizing the size distribution of the test nozzle. The droplets are allowed to freeze in flight and are collected for analysis. A simple sieve analysis of the collected wax particles, when fitted to a log-normal (bell shaped) distribution curve, yields the following key parameters that describe the nozzle spray product:

1. The mass mean particle diameter, $D_{m}$, a measure of the particle size produced by the nozzle. Fifty percent by weight (or volume) of the particle are larger and $50 \%$ are smaller than $D_{m}$.

2. The geometric standard deviation, $\sigma_{G}$, a measure of the breadth of the size distribution. $\sigma_{G}$ is the ratio: $D_{84.13 / D_{m}}$ (84.13\% by weight of the particles are smaller than $D_{84.13}$ ).

3. The correlation coefficient, a measure of the fit of the test data to a log-normal distribution curve.

A schematic diagram of the apparatus used for this study is shown in Figure 14.

Prior to a test, the wax reservoir is filled with preheated molten wax (about 2.5 Ib capacity) and the hot water tank surrounding the wax reservoir is filled with boiling water from the hot water supply tank. Hot water is continuously circulated from the supply tank on the ground level to the hot water tank overhead providing uniform heating and stabilization of the temperature in the wax reservoir and the reservoir discharge line including the two-way solenoid valve. Water from around the solenoid valve is also permitted to drain through a 1/8-in.-diameter orifice on the valve inlet to flush and help preheat the valve and discharge tubing when wax is not flowing. An electrical heating tape is used to heat the test nozzle mounted outside and below the hot water tank. 


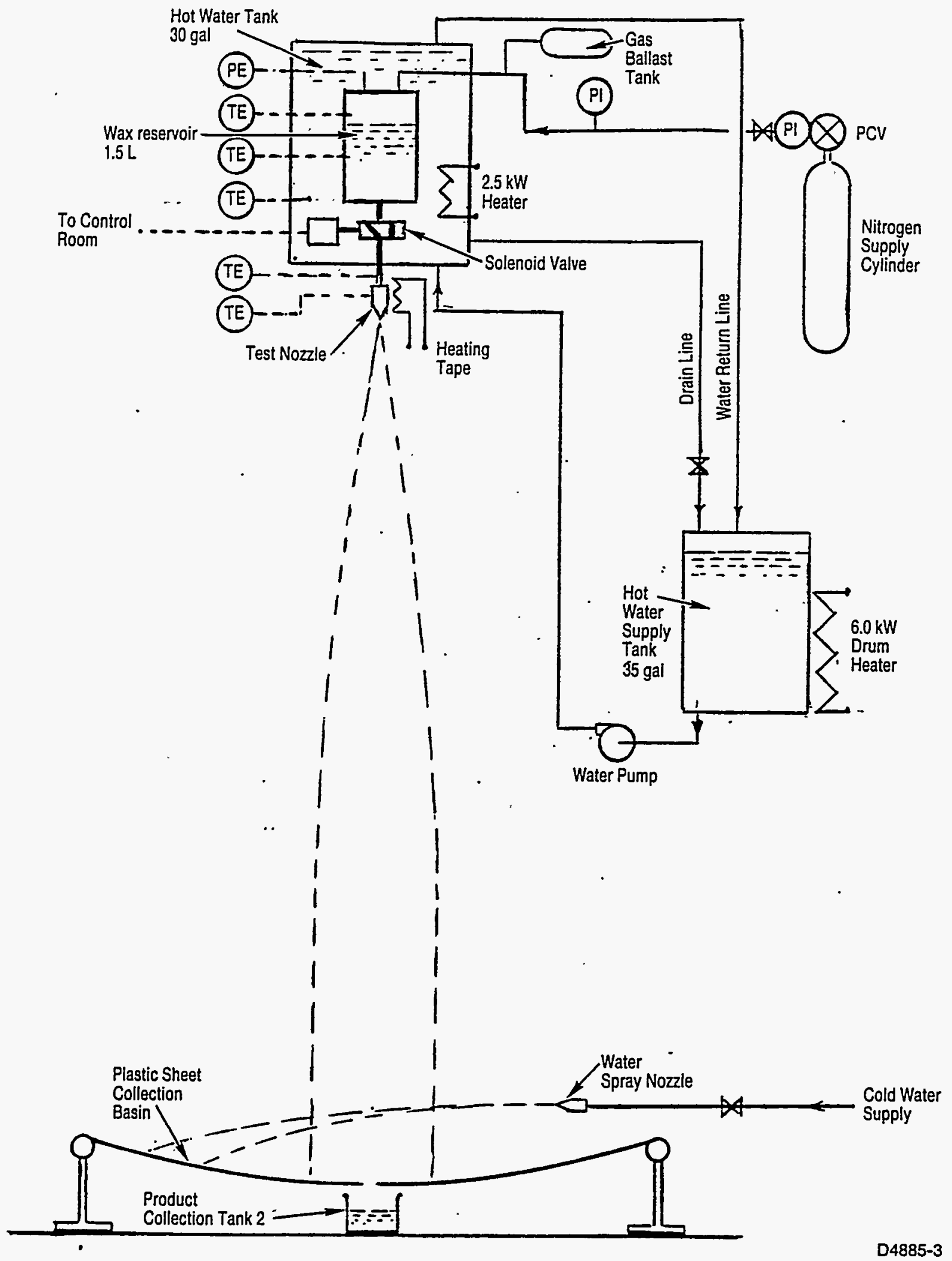

Figure 14. Schematic Diagram of Spray Nozzle Test Apparatus 
The test is initiated by pressurizing the wax reservoir to the desired test pressure using nitrogen, and then opening the solenoid valve for a predetermined time interval. The test nozzle discharges vertically downward approximately 10 to $11 \mathrm{ft}$ to a plastic sheet collection basin, which measures $10 \mathrm{ft} \times 10 \mathrm{ft}$. A horizontal spray of cold water is directed across the basin to aid in solidifying the hot wax droplets and flushing them through a hole in the center of the basin into a product collection tank. The entire quantity of wax sprayed is collected in water, separated from the water by filtration, and dried. The resulting dried wax product is weighed and sieved using six Tyler screens with aperture openings from 1,651 microns to 350 microns across.

The five temperature sensing elements (designated TE) shown in Figure 14 are used to measure the wax reservoir internal temperature (upper and lower), hot water tank internal temperature, wax temperature at the inlet to the test nozzle, and exterior skin temperature of the test nozzle. The pressure on the wax reservoir is set using a precision test gauge, $P I$, and monitored using a 0 - to $100-1 b / i^{2}$ (gage) pressure transducer, PE. An IBM XT computer coupled with a Keithly 500 Data Acquisition System (DAS) is used to record and display the temperature and pressure test measurements every $0.5 \mathrm{~s}$.

The hot-wax tests were conducted with Petrolite Polywax 500. This is a crystalline aliphatic hydrocarbon with a molecular weight of about 500 and a melting point of $187^{\circ} \mathrm{F} .{ }^{13}$ A comparison of the properties of the wax, water, and black liquor at typical spraying conditions for the three fluids is given in Table 8.

Table 8. Properties of Liquids

\begin{tabular}{llll}
\hline & $\begin{array}{c}\text { Polywax } \\
500\end{array}$ & $\begin{array}{c}\text { Black Liquor } \\
65 \% \text { Solids } \\
\text { (typical) }\end{array}$ & Water \\
\cline { 2 - 4 } Temperature, ${ }^{\circ} \mathrm{F}\left({ }^{\circ} \mathrm{C}\right)$ & $195(91)$ & $212(100)$ & $70(21)$ \\
Viscosity, $\mathrm{Cp}$ & 7.5 & 200 & 1.0 \\
Density, $\mathrm{g} / \mathrm{cc}$ & 0.83 & 1.34 & 1.0 \\
Surface tension, dyn/cm & 30 & 40 & 73 \\
\hline
\end{tabular}


The nozzles tested are listed in Table 9. They include three commercial nozzles and the new splash tube nozzle that was fabricated in Rocketdyne's Test Support Machine Shop.

Table 9. Nozzles Tested

\begin{tabular}{|c|c|c|c|c|}
\hline No. & Type & Manufacturer & Spray Pattern & Part Name/Number \\
\hline 1. & Pressure & $\begin{array}{l}\text { Spraying Systems } \\
\text { Company }\end{array}$ & Solid Cone-- $30^{\circ}$ & Fulljet 1/4 G3009 \\
\hline 2. & Pressure & $\begin{array}{l}\text { Spraying Systems } \\
\text { Company }\end{array}$ & Solid Cone-- $30^{\circ}$ & Fulljet $1 / 2$ G3030 \\
\hline 3. & Two Fluid & $\begin{array}{l}\text { North American } \\
\text { Manufacturing } \\
\text { Company }\end{array}$ & Two Fluid--30 & $\begin{array}{l}\text { Series } 5622 \text { O2A/O2B, } 0 i 1 \\
\text { Nozzle No. 3-3289-1, and } \\
\text { Steam Nozzle No. 3-3289-2 }\end{array}$ \\
\hline 4. & Pressure & $\begin{array}{l}\text { Experimental-- } \\
\text { Rocketdyne }\end{array}$ & Hollow Cone $--30^{\circ}$ & Splash Tube \\
\hline
\end{tabular}

In addition to the hot wax tests, tests were conducted on all of the above nozzles with cold water. These tests were used to observe the spray patterns and measure flow capacity data under various nozzle operating conditions. Flow capacity data were obtained by presetting the upstream nozzle pressure and collecting and weighing the total water stream emerging from the nozzle over a measured time interval.

\section{TEST RESULTS}

A total of 24 test runs were made with hot wax during this test series using the spray nozzles noted in Table 9. Flow and pressure data obtained with wax are presented in Table 10. The first test runs with the smaller (3009) full cone nozzle proved to be a series of checkout runs for the test apparatus and for refining the experimental techniques. 
Table 10. Summary of Flow/Pressure Data with Wax

\begin{tabular}{|c|c|c|c|c|c|c|c|c|c|}
\hline \multirow{2}{*}{$\begin{array}{l}\text { Run } \\
\text { No. }\end{array}$} & \multirow{2}{*}{$\begin{array}{l}\text { Nozzle le* } \\
\text { No. }\end{array}$} & \multirow{2}{*}{$\begin{array}{c}\text { Tank } \\
\text { Presşure } \\
\text { (1b/in2 } \\
\text { [gage]) }\end{array}$} & \multirow{2}{*}{$\begin{array}{l}\text { Nozzle } \\
\text { Presşure } \\
\text { (1b/inz } \\
\text { [gage]) }\end{array}$} & \multirow{2}{*}{$\begin{array}{l}\text { Nozzle } \\
\text { Temperature } \\
\left({ }^{\circ} \mathrm{F}\right)\end{array}$} & \multicolumn{2}{|c|}{$\begin{array}{l}\text { Wax Flow } \\
\text { (Caic) }\end{array}$} & \multicolumn{2}{|c|}{$\begin{array}{c}\text { Wax Flow } \\
\text { (Meas) }\end{array}$} & \multirow{2}{*}{$\begin{array}{l}\text { Air } \\
\text { Flow. } \\
\text { (Meas') }\end{array}$} \\
\hline & & & & & GPM & $\mathrm{lb} / \mathrm{h}$ & GPM & $\mathrm{lb} / \mathrm{h}$ & \\
\hline 1 & 1 & 15.7 & - & 198 & - & - & - & - & NA \\
\hline 2 & 1 & 49.1 & - & 188 & - & - & - & - & NA \\
\hline 3 & 1 & 48.8 & - & $=187$ & - & - & - & - & NA \\
\hline 4 & 1 & 36.7 & 33.5 & 194 & 0.898 & 373 & - & - & NA \\
\hline 4 & 1 & 15.0 & 13.7 & 192 & 0.574 & 239 & - & - & NA \\
\hline 6 & 1 & 48.2 & 44.0 & 192 & 1.03 & 428 & - & - & NA \\
\hline 7 & 1 & 49.0 & 44.7 & 194 & 1.04 & 431 & 0.487 & 203 & NA \\
\hline 8 & 1 & 23.4 & 21.4 & 194 & 0.717 & 298 & 0.376 & 156 & NA \\
\hline 9 & 2 & 47.9 & 26.2 & 195 & 2.34 & 973 & - & - & NA \\
\hline 10 & 2 & 57.3 & 31.4 & 191 & 2.56 & 1064 & - & - & NA \\
\hline $11 *$ & 2 & 9.97 & 5.46 & 195 & 1.07 & 444 & 1.25 & 520 & $N A$ \\
\hline 12 & 2 & 14.2 & 7.77 & 201 & 1.28 & 530 & 1.43 & 594 & NA \\
\hline 13 & 2 & 23.9 & 13.1 & 204 & 1.65 & 687 & - & - & $N A$ \\
\hline 14 & 3 & 4.37 & 1.94 & 204 & 0.785 & 326 & - & - & 51.2 \\
\hline 15 & 3 & 8.0 & 3.54 & 201 & 1.06 & 441 & - & - & 71.2 \\
\hline 16 & 3 & 9.20 & 4.08 & 200 & 1.14 & 473 & 1.24 & 516 & 46.8 \\
\hline 17 & 3 & 8.63 & 3.82 & 202 & 1.10 & 458 & 1.13 & 470 & 46.4 \\
\hline 18 & 3 & 8.76 & 3.88 & 200 & 1.11 & 462 & 1.52 & 632 & 46.4 \\
\hline 19 & 3 & 9.28 & 4.11 & 202 & 1.14 & 475 & - & - & 46.4 \\
\hline 20 & 3 & 8.87 & 3.93 & 202 & 1.12 & 465 & 1.28 & 531 & 37.1 \\
\hline 21 & 3 & 8.94 & 3.96 & 203 & 1.12 & 466 & 1.10 & 456 & 55.7 \\
\hline 22 & 4 & 24.3 & 11.8 & 203 & 1.78 & 740 & - & - & $N A$. \\
\hline 23 & 4 & 48.4 & 23.4 & 203 & 2.51 & 1044 & - & - & NA \\
\hline 24 & 4 & 72.6 & 35.1 & 203 & 3.08 & 1279 & - & - & NA \\
\hline
\end{tabular}

*Spray nozzles heated externally starting with Run 11 .

**See Table 9. 
An evaluation of the results for tests 1 through 10 indicated that the wax flow rates were lower than predicted on the basis of manufacturer's data with water. Two potential causes of the low flow rates were identified: (1) partial freezing of wax within the test nozzles was occurring and (2) the flow resistance of the wax flow path from the wax reservoir to the nozzle inlet was not insignificant.

Prior to Run 10, the test nozzles had been heated only by flowing hot water through them immediately before initiating wax flow. Water flow also occurred immediately after the test. Examination of the internals of the nozzles after each test showed them to be clear of wax; however, the flushing action of the hot water after the wax flow had stopped would have removed the evidence of flow blockage. As a precautionary measure, a heating tape was therefore installed on the test nozzles for all runs starting with number 11. A skin thermocouple was also installed to monitor and control the nozzle temperature.

A series of water flow capacity measurements was made on the test setup to determine if the wax flow path resistance was significant when compared to the flow resistance of the spray nozzles. The measurements indicated that the flow resistance in the line and solenoid valve could not be neglected. An accurate value of this was, therefore, measured using water resistance so that the wax reservoir pressure could be corrected to give nozzle inlet pressure as a function of flow rate.

A check of the measured wax flow rates versus the calculated flow rates was made with the nozzle heater in operation and using corrected pressure data. It was found that, rather than being about $50 \%$ low, the new flow rate measurements were approximately 10 to $15 \%$ higher than predicted. However, this result can be explained by the inherent error in timing the wax flow for the short discharge intervals required by the tests. Once the above modifications to the test setup and procedure were made, wax flow rate checks gave reasonable results.

AI-DOE- 13566 
Water flow capacity data were obtained on each of the test nozzles to determine throughput as a function of differential pressure. For low viscosity liquids (less than 100 to $200 \mathrm{cp}$ ), the nozzles should all behave like orifices with the volumetric flow rate affected only by pressure and density. This was born out by the consistent values of "K" observed for each nozzle. Table 11 presents the data obtained. The results for the commercial nozzles were within $10 \%$ of the data reported by the vendors.

The sieve analysis results on wax samples collected are presented in Table 12. Not all tests run were sieved. Examination of the wax collected during some runs revealed that a significant fraction of the particles were nonspherical; e.g., flattened out, or agglomerated and shapeless. These results generally occurred when low nozzle pressures were used; i.e., conditions that favored very large droplets. The large droplets were not cooled sufficiently in their 10- to 11-ft drop to the collection basin to form solid spheres. Figure 15 shows photographs of typical wax products from a run that produced predominately spherical particles (Run 6) and one that produced large nonspherical shapes (Run 22).

\section{DISCUSSION OF RESULTS}

The calculated drop size requirements for the pilot plant gasifier are summarized in Table 13. The values given are based on the calculation procedure described in the Phase $2 B$ Final Report. ${ }^{5}$ The minimum and maximum allowable drop sizes shown in the table are estimated by averaging the calculated results for the extreme cases of zero black liquor expansion and $100 \%$ expansion during the drying period.

No satisfactory method applicable to all types of nozzles has been developed for accurately extrapolating nozzle performance from one liquid to another. In general, the drop size can be expected to increase with increased viscosity and increased surface tension and decrease with decreased density. For pressure nozzles, available correlations indicate that water and hot wax will give about the same size drops, while black liquor (at $100^{\circ} \mathrm{C}$ ) will, yield drops which are 40 to $100 \%$ larger.

AI-DOE-13566 
Table 11. Water Capacity Data

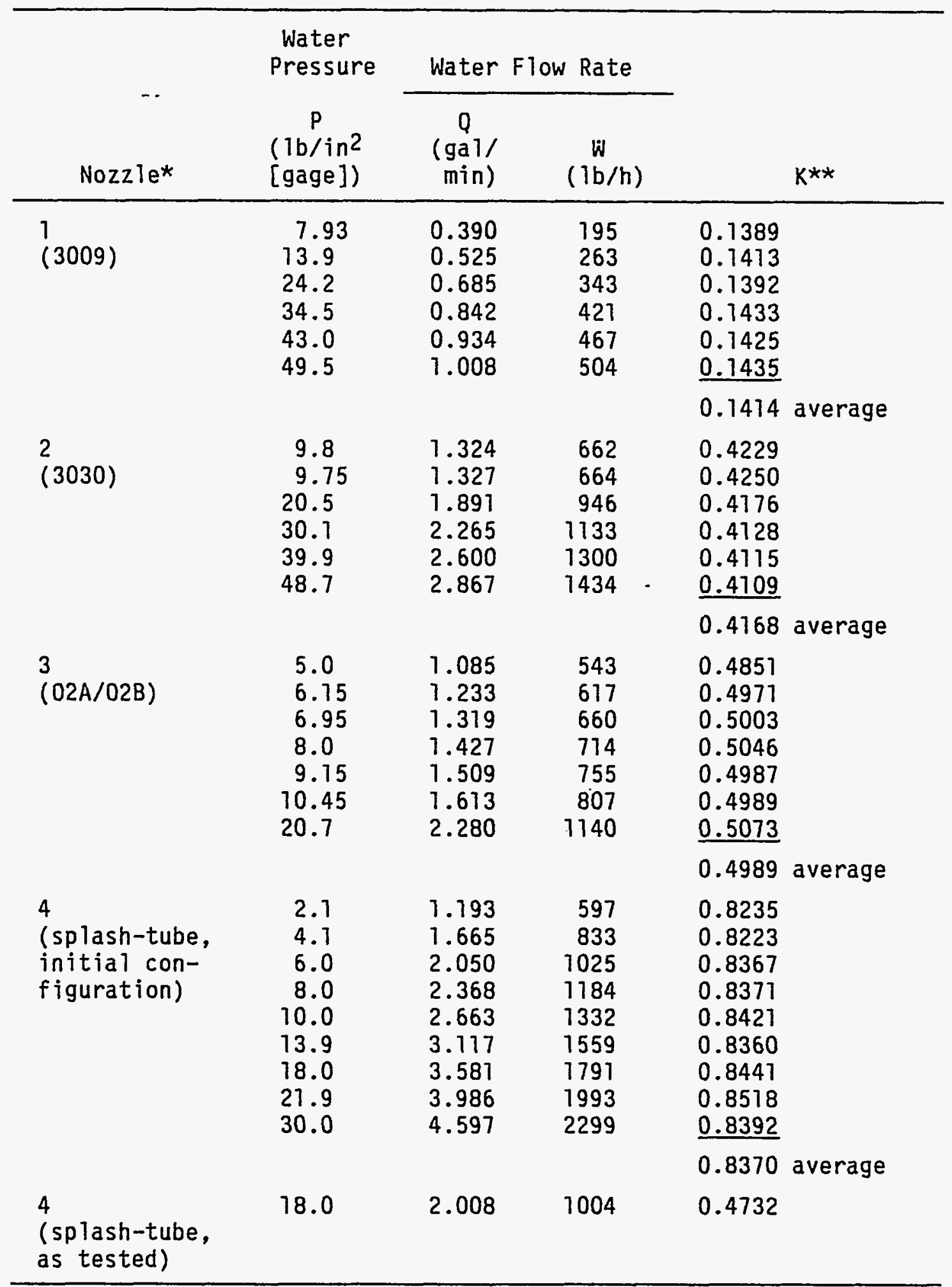

*See Table 9.

$*_{K}=Q\left(p_{L} / P\right)^{T / 2}$ 
Table 12. Size Distribution Results with Hot Wax

\begin{tabular}{|c|c|c|c|c|c|c|c|c|c|c|}
\hline \multirow[b]{3}{*}{ Nozzle* } & \multicolumn{10}{|c|}{ Run Number } \\
\hline & 4 & 6 & 10 & 14 & 15 & 19 & 20 & 21 & 23 & 24 \\
\hline & 1 & 1 & 2 & 3 & 3 & 3 & 3 & 3 & 4 & 4 \\
\hline \multicolumn{11}{|l|}{ Sieve Data } \\
\hline \multicolumn{11}{|l|}{ Weight per screen, $g$} \\
\hline 1651 microns & 0.923 & 1.360 & 12.208 & 5.624 & 14.320 & 6.927 & 8.701 & 3.303 & 16.199 & 16.252 \\
\hline 1410 & 3.422 & 0.887 & 4.984 & 2.126 & 2.345 & 2.471 & 3.926 & 1.788 & 3.563 & 3.691 \\
\hline 1000 & 3.902 & 7.238 & 21.077 & 5.232 & 4.378 & 5.211 & 8.419 & 4.302 & 7.139 & 7.932 \\
\hline 840 & 19.764 & 23.607 & 14.845 & 4.870 & 4.096 & $4.40\}$ & 6.513 & 3.568 & 4.146 & 5.006 \\
\hline 425 & 10.164 & 59.037 & 30.225 & 18.161 & 17.200 & 14.509 & 21.431 & 14.456 & 12.450 & 13.801 \\
\hline 350 & 25.142 & 8.380 & 8.041 & 5.979 & 6.620 & 5.085 & 6.095 & 5.143 & 3.031 & 2.780 \\
\hline PAN & 9.038 & 14.614 & 7.019 & 43.298 & 42.449 & 24.457 & 35.209 & 21.729 & 7.200 & 6.872 \\
\hline Total & 72.355 & 115.123 & 98.399 & 85.390 & 91.408 & 63.061 & 90.294 & 54.289 & 53.728 & 56.334 \\
\hline \multicolumn{11}{|l|}{ Calculated Results ${ }^{* *}$} \\
\hline \multicolumn{11}{|l|}{ Normalized $\%$ undersize } \\
\hline 1651 microns & 93.995 & 98.819 & 87.593 & 93.414 & 84.334 & 89.015 & 90.364 & 93.916 & 69.850 & $71.15 !$ \\
\hline 1410 & 88.602 & 98.048 & 82.528 & 90.924 & 81.769 & 85.097 & 86.016 & 90.622 & 63.218 & 64.599 \\
\hline 1000 & 61.287 & 91.761 & 61.108 & 84.797 & 76.979 & 76.834 & 76.692 & 82.698 & 49.931 & 50.518 \\
\hline 840 & 47.239 & 71.255 & 46.022 & 78.976 & 72.498 & 69.855 & 69.479 & 76.126 & 42.214 & 41.632 \\
\hline 425 & 12.491 & 19.973 & 15.305 & 57.708 & 53.681 & 46.847 & 45.744 & 49.498 & 19.042 & 17.134 \\
\hline 350 & - & 12.694 & 7.133 & 50.706 & 46.439 & 38.783 & 38.994 & 40.025 & 13.401 & 12.199 \\
\hline \multirow[t]{2}{*}{ Mass mean diameter, microns } & 805.51 & 598.16 & 831.82 & 347.99 & 379.44 & 470.51 & 476.49 & 437.27 & 1007.33 & 1001.62 \\
\hline & \pm 27.35 & \pm 20.49 & \pm 16.78 & \pm 6.22 & \pm 14.84 & \pm 6.84 & \pm 11.82 & \pm 6.28 & \pm 5.82 & \pm 4.42 \\
\hline \multirow[t]{2}{*}{ Geometric standard dev. } & 1.625 & 1.538 & 1.821 & 2.837 & 4.087 & 2.834 & 2.699 & 2.403 & 2.635 & 2.463 \\
\hline & \pm 0.042 & \pm 0.035 & \pm 0.038 & \pm 0.052 & \pm 0.236 & \pm 0.054 & \pm 0.085 & \pm 0.036 & \pm 0.024 & \pm 0.016 \\
\hline Correlation coefficient & 0.9943 & 0.9945 & 0.9975 & 0.9994 & 0.9967 & 0.9993 & 0.9980 & 0.9994 & 0.9998 & 0.9999 \\
\hline
\end{tabular}

*See Table 9.

**Based on least squares fit of test data to log-normal distribution curve.

$16070 /$ tab 
$995 \varepsilon L-300-I \forall$

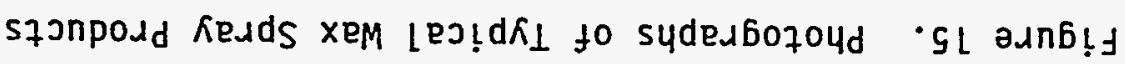

¿2 uny wodf zonpodd pana!sun fo aldwes ( $q$

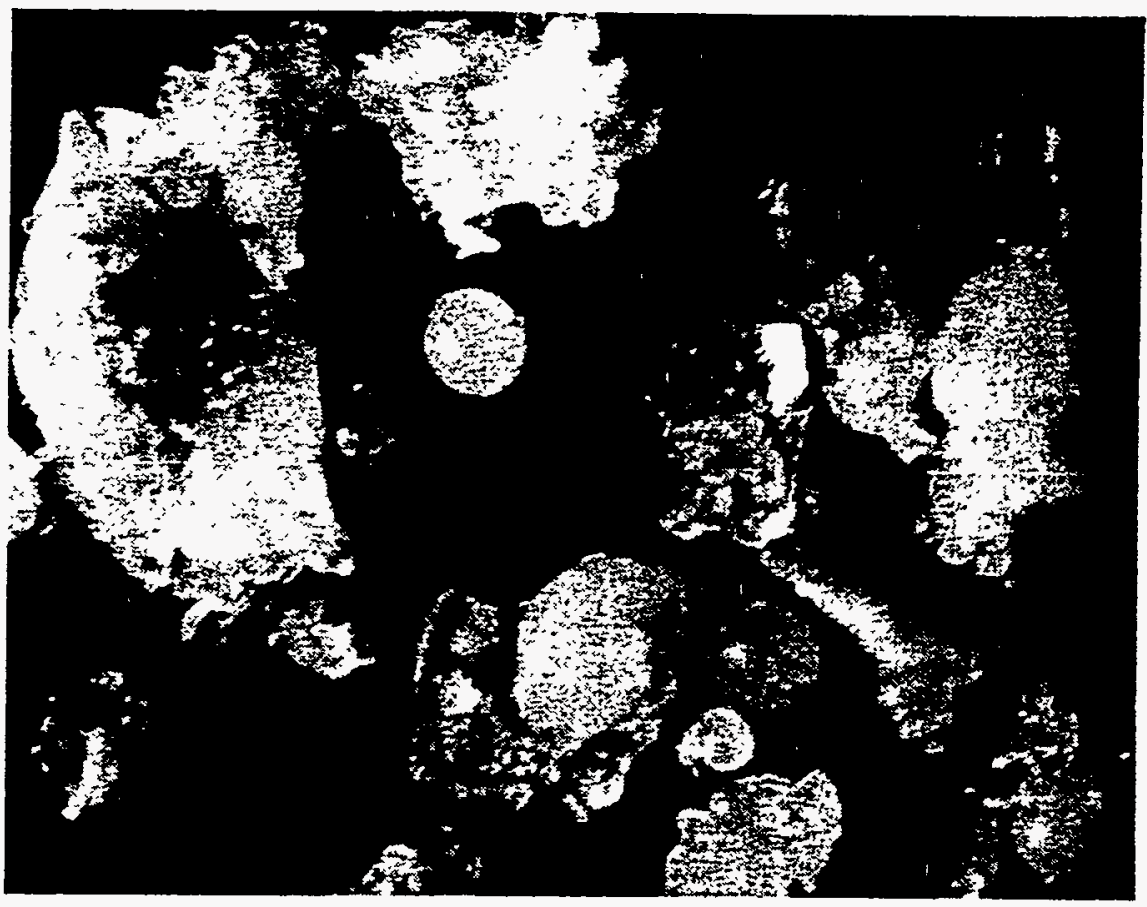

9 uny wodf zonpodd pana!sun to əldwes (

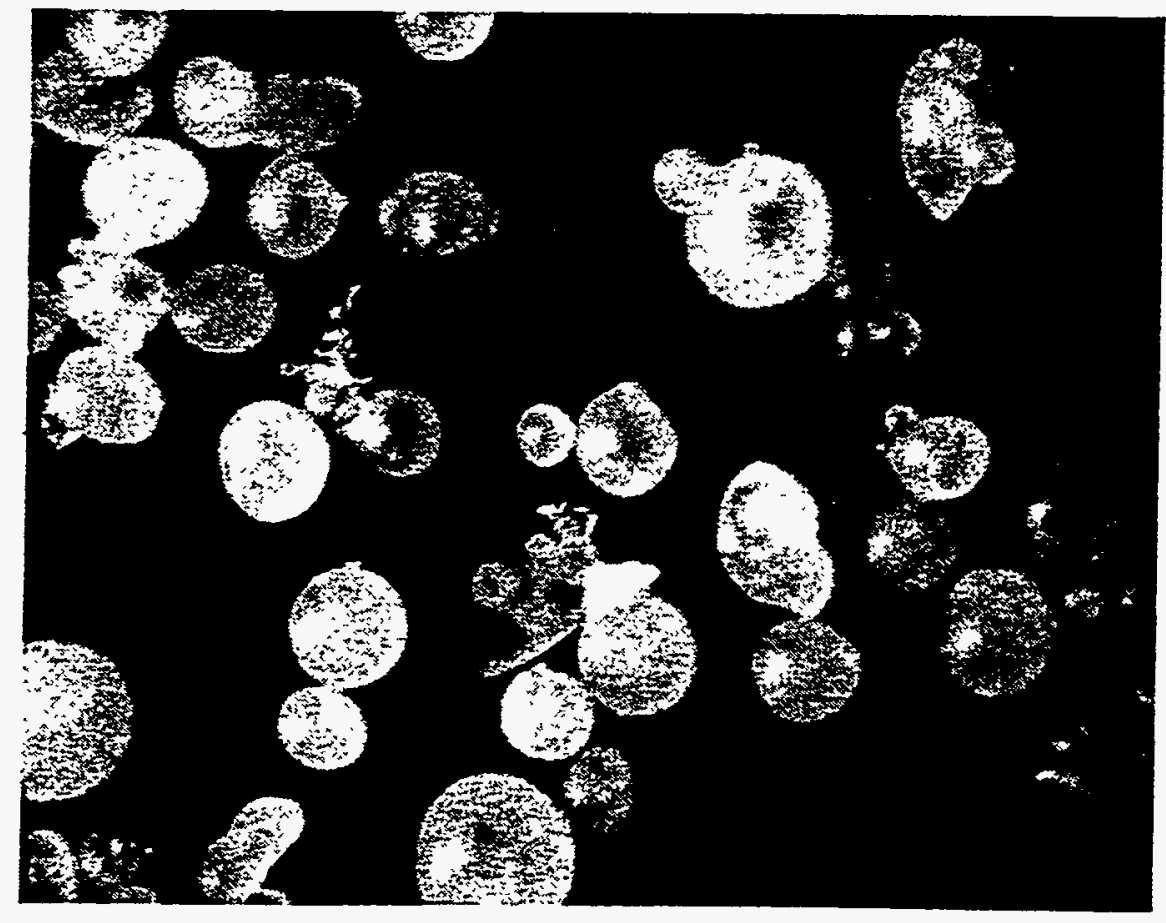


Table 13. Black Liquor Spray Requirements

\begin{tabular}{lccc}
\hline & \multicolumn{3}{c}{ Design Case } \\
\cline { 2 - 5 } & A & B & C \\
\cline { 2 - 5 } Black liquor feed rate, 1b/h & 391 & 982 & 2,068 \\
Required drop size* & & & \\
Minimum size, microns & 300 & 400 & 500 \\
Maximum size, microns & 1,300 & 1,700 & 2,200 \\
Dm & 600 & 800 & 1,100 \\
\hline *Approximate values based on average of drop sizes calcu- \\
lated by assuming zero and 100\% expansion of sprayed black \\
liquor drops during drying.
\end{tabular}

The hot wax drop size results for the four nozzles tested are summarized in Table 14. Typical size distribution curves for three different nozzle types are given in Figure 16.

For the specific nozzles and flow rates tested, the solid cone pressure nozzles appeared to give the particle size nearest to the desired value and also the narrowest size range. However, it was observed that this nozzle ejects the liquid with a very high downward velocity. In the wax spraying operations, this limited the tests to conditions which produced relatively sinall drops because the large drop sprays apparently did not have time to harden prior to collection. In the gasifier application, the high downward velocity would limit the time available for drying.

The two-fluid nozzle using hot wax and air was observed to develop a mist of very small particles in addition to the main stream of medium size drops. This nozzle also showed the greatest breadth of size distribution for the particles collected and sieved. The main drawback of the two-fluid nozzle, however, is its large requirement for air or steam. The minimum amount of gas which will produce a relatively stable spray is one-tenth the amount of liquid by weight. In a gasifier processing black liquor containing $35 \%$ water, the use of $0.1 \mathrm{lb}$ of steam per pound of feed would increase the water content of 
Table 14. Summary of Hot Wax Spray Test Results

\begin{tabular}{|c|c|c|c|c|c|c|c|}
\hline $\begin{array}{l}\text { Run } \\
\text { No. }\end{array}$ & $\begin{array}{c}\text { Nozzle } \\
\text { No.a }\end{array}$ & Identification & $\begin{array}{l}\text { Wax } \\
\text { Pressure } \\
\left(1 \mathrm{~b} / \mathrm{in}^{2}\right)\end{array}$ & $\begin{array}{l}\text { Wax } \\
\text { Flow Rate } \\
(1 \mathrm{~b} / \mathrm{h})\end{array}$ & $\begin{array}{l}\text { Air } \\
\text { Flow Rate } \\
(1 \mathrm{~b} / \mathrm{h})\end{array}$ & $\begin{array}{c}D^{C} \\
\text { (micloons) }\end{array}$ & $\sigma_{G}{ }^{d}$ \\
\hline 4 & 1 & Fulljet 3009 & $33: 5$ & $<373$ & - & 806 & 1.6 \\
\hline 6 & 1 & Fulljet 3009 & 44.0 & $<428$ & - & 598 & 1.5 \\
\hline 10 & 2 & Fulljet 3030 & 31.4 & $<1,064$ & - & 832 & 1.8 \\
\hline 14 & 3 & NA $02 A / 02 B$ & 1.9 & 326 & 51.2 & 348 & 2.8 \\
\hline 15 & 3 & $N A \quad 02 A / 02 B$ & 3.5 & 441 & 71.2 & 379 & 4.7 \\
\hline 19 & 3 & NA $02 A / 02 B$ & 4.1 & 475 & 46.4 & 471 & 2.8 \\
\hline 20 & 3 & $N A 02 A / 02 B$ & 3.9 & 465 & 37.1 & 476 & 2.7 \\
\hline 21 & 3 & $N A 02 A / 02 B$ & 4.0 & 466 & 55.7 & 437 & 2.4 \\
\hline 23 & 4 & Splash tube & 23.4 & 1,044 & - & 1,007 & 2.6 \\
\hline 24 & 4 & Splash tube & 35.1 & 1,279 & - & 1,002 & 2.5 \\
\hline
\end{tabular}

aSee Table 9.

bCalculated on the basis of water flow tests. Actual rates for Runs 4, 6, and 10 probably less than calculated values due to possible wax cooling and solidification within the nozzles.

CMass mean diameter $=D_{m}$.

$\mathrm{d}_{\text {Geometric standard deviation }}=\sigma_{\mathrm{G}}$.

the product gas by almost $30 \%$ and would represent a significant consumption of process steam.

The splash-tube nozzle appeared to give an excellent spray pattern and the wax drops left the nozzle with a relatively low downward velocity. The observed drop sizes for the unit tested are somewhat larger then desired, but probably similar to those of a solid cone pressure nozzle with the same capacity (i.e., $1,279 \mathrm{lb} / \mathrm{h}$ at $35.1 \mathrm{psi}$ ). In general, the drop size of pressure nozzles increases with increased nozzle capacity (orifice size) and, for a given nozzle, decreases with increased pressure drop. The results of Runs 23 and 24 indicate that the splash-tube nozzle differs from conventional pressure nozzles in giving an almost constant drop size with changing pressure (and throughput). It is anticipated that smaller (or larger) drops can be obtained with this type of nozzle by changing the orifice size and splash tube configuration. 


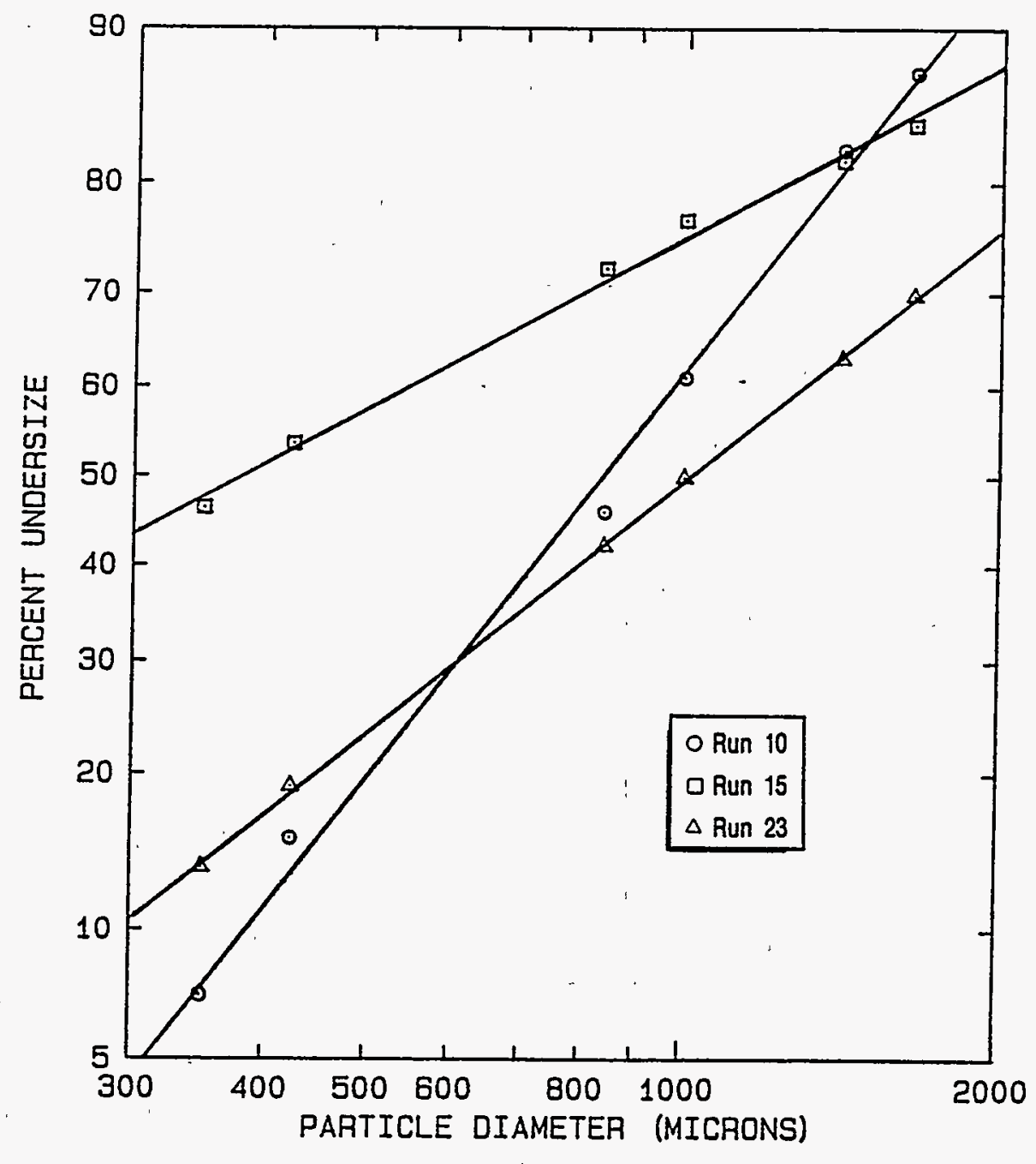

D4885-2

Figure 16. Typical Size Distribution Curves for Spray Products 
The results of the spray tests and published data indicate that all three types of nozzles can be designed and operated to give black liquor drops in the desired size range. The two-fluid nozzle is considered to be undesirable because of its high consumption of steam. The solid-cone and splash-tube nozzles are recommended for testing with black liquor in conjunction with the pilot plant program.

The splash-tube design is preferred at this time over the solid-cone nozzle because it does not contain a vane insert that may be subject to plugging by black liquor solids; it provides a lower exit velocity; and it can be cleaned and/or adjusted while in service.

$16070 / \mathrm{j} 1 \mathrm{~m}$ 
AI-DOE-13566 


\section{CONCLUSIONS AND RECOMMENDATIONS}

The black liquor gasification pilot plant design has been upgraded and improved to increase its operating pressure and capacity by a factor of 2 (to $90 \mathrm{lb} / \mathrm{in}^{2}$ (absolute) and $2,068 \mathrm{lb} / \mathrm{h}$ black liquor feed, respectively). The capacity increase causes the predicted heating value of the product gas to increase from 101 to $114 \mathrm{Btu} / \mathrm{scf}$ (dry basis).

All major pilot plant design uncertainties have been resolved and the required hardware and systems have been defined in sufficient detail to provide a firm basis for proceeding with the final design, construction, and operation of the facility.

No significant problems have been uncovered during the pilot plant design activities which would preclude satisfactory operation or attainment of the key design objectives of producing gas with a higher heating value (HHV) of . $100 \mathrm{Btu} / \mathrm{scf}$ and melt in which over $90 \%$ of the sulfate is reduced to sulfide.

It is concluded that the black liquor gasification pilot plant as now configured represents a well-integrated, cost-effective facility for demonstrating all critical operations of the process and providing data for the design of much larger units.

Tests of three types of nozzles with hot wax indicated that all three can be designed and operated to spray black liquor into the gasifier with the desired drop size distribution and narrow cone pattern. One type, a two-fluid (steam/black liquor) nozzle, has been removed from further consideration because of $i$ ts indicated high consumption of steam.

It is recommended that two of the nozzles, a narrow-angle, full-cone design and a specially designed splash-tube concept, be tested with black liquor as part of the pilot plant experimental program and that the final reference design be selected on the basis of the results. 
The splash-tube nozzle is preferred at this time because it is less subject to plugging (the full-cone nozzle contains internal vanes); ejects the black liquor with a lower initial downward velocity; and can be cleaned and/or adjusted while in service. However, the full-cone nozzle design warrants further consideration as a backup design because it provides a solid-cone rather than hollow-cone spray pattern and appears to give a somewhat narrower size distribution.

1607D/j1m 


\section{REFERENCES}

1. Kelleher, E. G., "Feasibility of Black Liquor Gasification in Combined Cycle Cogeneration," Final Report - Phase 1, DOE/CS/40341-T4 (June 30, 1983)

2. Kohl, A. L., Barclay, K. M., Stewart, A. E., and Estes, G. R., "Black Liquor Gasification," Phase 2 Final Report, DE85013798 (November 1984)

3. Kohl, A. L., Stewart, A. E., and Estes, G. R., "Black Liquor Gasification," Phase 2A Final Report AI-DOE-13521 (June 1985)

4. Kelleher, E. G., "Gasification of Kraft Black Liquor and Use of the Products in Combined Cycle Cogeneration," F.inal Report - Phase II, DOE/CS/40341-T5 (JuTy 31, 1985)

5. Kohl, A. L., Stewart, A. E., Estes, G. R., Barclay, K. M., and Heredy, L. A., "Black Liquor Gasification," Phase 2B Final Report AI-DOE-13546 (May 1986)

6. Koh1, A. L., and Stewart, A. E., "Black Liquor Gasification Phase 2C Final Report AI-DOE-13560 (March 1987)

7. Kèlleher, E. G., "Conceptual Design of a Black Liquor Gasification Pilot Plant," work performed under US DOE Contract No. DE-AC05-80CS40341 (August 1987)

8. Sarraf, R. J., "Corrosion Testing of Construction Materials in AI Coal Gasification Process Green Liquor," Rockwell Supporting Document N423-TR-230-002 issued October 1, 1975.

9. Perry, R. H. and Chilton, C. H., editors, "Chemical Engineers Handbook," Fifth Edition, MCGraw-Hi11 Book Company, New York, 1973, Pages 5-31 and $5-32$

10. Kim, K. Y. and Marshall, W. R., Jr., "Drop-Size Distribution from Pneumatic Atomizers," AI Che. Journal, Vol. 17, No. 3 (May 1971), p 575

11. Dickerson, R., et al., "Correlation of Spray Injector Parameters with Rocket Engine Performance," Rocketdyne Technical Report AFRPL-TR-68-147 (June 1968)

12. Joyce, J. R., "The Wax Method of Spray Particie Size Measurement," Technical Report No. I.C.T.17, Shell Petroleum Co. Ltd., Norman House, Strand, London

13. Petrolite Corporation Technical Data Release No. BP 100, "Polywax® Polyethylenes," November 1981

16070/jim

AI-DOE-13566 